GENERALIZED LITTLEWOOD-RICHARDSON COEFFICIENTS FOR BRANCHING RULES OF GL $(N)$ AND EXTREMAL WEIGHT CRYSTALS

\author{
A Dissertation \\ presented to \\ the Faculty of the Graduate School \\ University of Missouri
}

In Partial Fulfillment

of the Requirements for the Degree

Doctor of Philosophy

by

BRETT COLLINS

Dr. Calin Chindris, Dissertation Supervisor

MAY, 2018 
The undersigned, appointed by the Dean of the Graduate School, have examined the dissertation entitled

\section{GENERALIZED LITTLEWOOD-RICHARDSON COEFFICIENTS FOR BRANCHING RULES OF GL $(N)$ AND EXTREMAL WEIGHT CRYSTALS}

presented by Brett Collins, a candidate for the degree of Doctor of Philosophy of Mathematics, and hereby certify that in their opinion it is worthy of acceptance.

Professor Calin Chindris

Professor Ian Aberbach

Professor Dan Edidin

Professor David Retzloff 


\section{ACKNOWLEDGEMENTS}

Much like how a statue is primarily made by chipping away small pieces rather than by breaking off large chunks, a person is greatly shaped and affected by every interaction with another, no matter how small or seemingly inconsequential the action might be. With this in mind, undoubtedly I will forget to thank some people for helping me become who I am and for making this thesis possible. In an attempt to rectify this, I want to thank now all the people who have been a part of my life, both in the MU math department and outside too.

In particular, I am deeply grateful to my advisor, Calin Chindris, for teaching me quiver representation theory, his suggestion of the topic of this thesis and his continuous guidance throughout its writing, and for always being a genuinely supportive and positive person.

I am thankful for the professors I've had while at Mizzou, especially Ian Aberbach and Dan Edidin, with whom I've had over half of my graduate classes. They have played an essential role in making me the algebraist I am.

My heartfelt thanks to my graduate student friends, especially my academic siblings Dan Kline, Valerie Granger, Killian Meehan, and David Meyer. Their support, discussions, and career advice broadened my mathematical interests and greatly enriched my graduate life.

This thesis would not have been possible without my parents, whose support and encouragement have guided me in life. They are undoubtedly the ones responsible for my interest in math and continue to share in that interest, from dot cards and toy calculators to learning the phrase "Quiver Representation Theory" in order to 
impress others with their son's work.

Finally, a special thanks to my cat Myshkin, whose furry comfort and frequent scratches have provided a needed relief from daily math while in graduate school. Any shortcomings in this thesis are due to his welcomed distractions and poor editing skills. 


\section{Contents}

$\begin{array}{ll}\text { Acknowledgements } & \text { ii }\end{array}$

Abstract vii

1 Introduction 1

1.1 Background and motivation $\ldots \ldots \ldots \ldots \ldots$

1.1 .1 Horn's conjecture .................... 1

1.1.2 Stretched polynomials and Geometric Complexity Theory . . . 6

1.1 .3 Branching rules ...................... 9

1.2 Main results . . . . . . . . . . . . . . . . . . . . . 12

1.3 Organization of the thesis . . . . . . . . . . . . 15

$\begin{array}{llr}2 & \text { Background material } & 17\end{array}$

2.1 Representation theory of $\mathrm{GL}(n) \ldots \ldots \ldots \ldots \ldots$

2.1 .1 Schur modules . . . . . . . . . . . . . . . . . . . . 19

2.1.2 The complexity of Littlewood-Richardson coefficients . . . . . . 31

2.2 Quiver theory . . . . . . . . . . . . . . . . . 37

2.2.1 Preliminaries . . . . . . . . . . . . . . . 37

2.2 .2 Semi-invariants for quivers . . . . . . . . . . . . 38

$2.2 .3 \quad \sigma$-semi-stability $\ldots \ldots \ldots \ldots \ldots \ldots$ 
2.2.4 The facets of the cone of effective weights . . . . . . . . 46

2.2.5 Matrix equations and moment maps . . . . . . . . . . . 52

3 A quiver interpretation of generalized Littlewood-Richardson coef-

ficients $\quad 54$

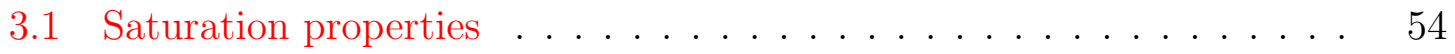

3.1 .1 Sun quiver $\ldots \ldots \ldots \ldots \ldots \ldots \ldots \ldots \ldots \ldots$

3.1 .2 Generalized star quiver . . . . . . . . . . . . . . . . 61

3.2 The facets of the cones of effective weights . . . . . . . . 64

3.3 Horn-type inequalities . . . . . . . . . . . . . . . . . 68

3.3 .1 Sun quiver $\ldots \ldots \ldots \ldots \ldots \ldots \ldots \ldots \ldots$

3.3 .2 Generalized star quiver . . . . . . . . . . . . . 76

3.4 Generalized eigenvalue problems . . . . . . . . . . . . 81

3.4.1 Generalized eigenvalue problem for $f_{1} \ldots \ldots \ldots \ldots 2$

3.4.2 Generalized eigenvalue problem for $f_{2} \ldots \ldots \ldots \ldots$

4 The combinatorics and complexity of generalized Littlewood-Richardson $\begin{array}{ll}\text { coefficients } & 90\end{array}$

4.1 Factorization formulas . . . . . . . . . . . . . . . . . . 90

4.2 Level-1 weights and stretched polynomials . . . . . . . . . . . . 93

4.2.1 Level-1 weights. . . . . . . . . . . . . . . . . . . . 93

4.2 .2 Stretched weights $\ldots \ldots \ldots \ldots \ldots \ldots \ldots$

4.3 Polytopal description and complexity . . . . . . . . . . . . . 102

4.3 .1 Polytopal description . . . . . . . . . . . . . . . . . . 102

Appendix . . . . . . . . . . . . . . . . . . . . . 113 
Index of symbols . . . . . . . . . . . . . . . . . . 116

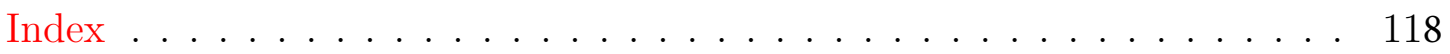

Bibliography . . . . . . . . . . . . . . . . . . . . . . 120

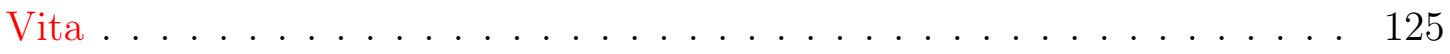




\begin{abstract}
This thesis is devoted to the combinatorial and geometric study of certain multiplicities, which we call generalized Littlewood-Richardson coefficients. These are sums of products of single Littlewood-Richardson coefficients, and the specific ones we study describe the branching rules for the direct sum and diagonal embeddings of $\operatorname{GL}(n)$ as well as the decompositions of extremal weight crystals of type $A_{+\infty}$. By representing these multiplicities as dimensions of weight spaces of quiver semi-invariants, we use quiver theory to prove their saturation and describe necessary and sufficient conditions for them to be nonzero, culminating in statements similar to Horn's classical conjecture. We then use these conditions to prove various combinatorial properties, including how these multiplicities can be factored and that these numbers in certain cases satisfy the same conjectures as single Littlewood-Richardson coefficients. Finally, we provide a polytopal description of these multiplicities and prove that their positivity can be computed in strongly polynomial time.
\end{abstract}




\section{Chapter 1}

\section{Introduction}

\subsection{Background and motivation}

\subsubsection{Horn's conjecture}

The inspiration for the results in this thesis comes originally from a problem of $\mathrm{H}$. Weyl [Wey12]. In 1912, motivated by problems in solid mechanics, Weyl asked for a description of the eigenvalues of a sum of Hermitian matrices in terms of those of the summands. Specifically, if $H(1), H(2)$, and $H(3)$ are $n \times n$ matrices, then because it is a standard result in linear algebra that the eigenvalues of a Hermitian matrix are real-valued, their eigenvalues may be written in weakly decreasing order:

$$
\lambda(i): \quad \lambda_{1}(i) \geqslant \lambda_{2}(i) \geqslant \ldots \geqslant \lambda_{n}(i), \quad i \in\{1,2,3\} .
$$

Weyl's eigenvalue problem may then be phrased as follows:

Weyl's eigenvalue problem. Let $\lambda(i)$ denote a weakly decreasing sequence of $n$ real numbers. Describe the triples $(\lambda(1), \lambda(2), \lambda(3))$ for which there exist $n \times n$ Hermitian matrices $H(1), H(2), H(3)$ with eigenvalues $\lambda(1), \lambda(2)$, and $\lambda(3)$, respectively, such that

$$
H(2)=H(1)+H(3) .
$$

One obvious necessary condition on the eigenvalues arises from taking the traces 
of both sides of the equation:

$$
\sum_{i=1}^{n} \lambda_{i}(2)=\sum_{i=1}^{n} \lambda_{i}(1)+\sum_{i=1}^{n} \lambda_{i}(3)
$$

It is clear, however, that this trace equation is far from sufficient.

Several important necessary inequalities were found by Weyl, Fan, Lidskii, Wielandt, and others in the next 50 years (see [Ful97b] for a survey of the history and results pertaining to this problem), culminating in a systematic study of the necessary and sufficient inequalities by A. Horn [Hor62]. Horn's extraordinary conjecture not only precisely stated the inequalities for the solution to the problem, it also proposed a recursive formula for checking if a triple $(\lambda(1), \lambda(2), \lambda(3))$ is in the solution set.

The first major step in proving the conjecture was made when A. Klyachko [Kly98] found necessary and sufficient homogeneous linear inequalities for the eigenvalues. In the same paper, Klyachko made the connection between these inequalities and Littlewood-Richardson coefficients using Schubert calculus. In order to precisely state Klyachko's result and related results, we first need to define some notation.

We call a weakly decreasing sequence of integers $\lambda=\left(\lambda_{1}, \ldots, \lambda_{n}\right)$ a partition if $\lambda_{n} \geqslant 0$, and in this case say it has at most $n$ nonzero parts, or length at most $n$. If $I=\left\{z_{1}<z_{2}<\ldots<z_{r}\right\}$ is an $r$-tuple of positive integers, define $\lambda(I)=\left(z_{r}-r, \ldots z_{1}-1\right)$. Note that this will always be a partition. For a complex vector space $V$ of dimension $n$ and a weakly decreasing sequence of $n$ integers $\lambda=\left(\lambda_{1}, \ldots, \lambda_{n}\right)$, we denote the irreducible rational representation of $\mathrm{GL}(V)$ with highest weight $\lambda$ as $S^{\lambda} V$; see Section 2.1 for the construction. Given any three weakly decreasing sequences of $n$ integers $\lambda(1), \lambda(2), \lambda(3)$, the Littlewood-Richardson coefficient $c_{\lambda(1), \lambda(3)}^{\lambda(2)}$ is defined to be

$$
c_{\lambda(1), \lambda(3)}^{\lambda(2)}=\operatorname{dim}_{\mathbb{C}} \operatorname{Hom}_{\mathrm{GL}(V)}\left(S^{\lambda(2)}(V), S^{\lambda(1)}(V) \otimes S^{\lambda(3)}(V)\right),
$$


that is, the multiplicity of $S^{\lambda(2)}(V)$ in $S^{\lambda(1)}(V) \otimes S^{\lambda(3)}(V)$. If $\lambda=\left(\lambda_{1}, \ldots, \lambda_{n}\right)$ is any sequence of $n$ real numbers, define $|\lambda|=\sum_{i=1}^{n} \lambda_{i}$ and $N \lambda=(N \lambda(1), \ldots, N \lambda(n))$.

Klyachko claimed that his stated inequalities were independent, but S. Agnihotri and C. Woodward [AW98] showed many inequalities to be redundant, and later P. Belkale [Bel01] showed that all inequalities for which $c_{\lambda\left(I_{1}\right), \lambda\left(I_{3}\right)}^{\lambda\left(I_{2}\right)}>1$ are redundant, which includes the list found by Agnihotri and Woodward. The remaining inequalities would be irredundant by a theorem of Klyachko [Kly98] provided the saturation of Littlewood-Richardson coefficients.

Theorem 1.1 (Saturation conjecture). For weakly decreasing sequences of $n$ integers $\lambda(1), \lambda(2)$, and $\lambda(3), c_{N \lambda(1), N \lambda(3)}^{N \lambda(2)} \neq 0$ for some positive $N$ if and only if $c_{\lambda(1), \lambda(3)}^{\lambda(2)} \neq 0$.

The statement that $c_{\lambda(1), \lambda(3)}^{\lambda(2)} \neq 0$ implies $c_{N \lambda(1), N \lambda(3)}^{N \lambda(2)} \neq 0$ for all positive integers $N$ follows immediately from the Littlewood-Richardson rule (Proposition 2.17), by these multiplicities forming a semigroup (see [Zel99]), or as a consequence of the Borel-Weil Theorem. A. Knutson and T. Tao [KT99] proved the converse by using combinatorial gadgets called honeycombs and hive models, completing the proof of Horn's conjecture. A similar proof of the conjecture using only hive models is found in [Buc00], while P. Belkale [Bel06] provided a geometric proof of the saturation conjecture using Schubert calculus. More importantly for this thesis, H. Derksen and J. Weyman proved the saturation conjecture for Littlewood-Richardson coefficients in [DW00a] by using results from quiver theory. The motivation of their proof comes from a result with A. Schofield [DSW07] where they showed that the number of subrepresentations of a specific dimension of a given dimension vector for an acylic quiver may be determined using Schubert calculus. With this reasoning, Derksen and 
Weyman defined a specific quiver and dimension vector for which this number was a given Littlewood-Richardson coefficient. In this way, they were able to extend results about Littlewood-Richardson coefficients to the more general setting of quiver theory.

Theorem 1.2 ([Kly98], [KTW04], [DW00a]). Let $\lambda(i)=\left(\lambda_{1}(i), \ldots, \lambda_{n}(i)\right), i \in\{1,2,3\}$, be weakly decreasing sequences of $n$ real numbers. Then the following are equivalent:

1. there exist $n \times n$ complex Hermitian matrices $H(i)$ with eigenvalues $\lambda(i)$ such that

$$
H(2)=H(1)+H(3) ;
$$

2. the numbers $\lambda_{j}(i)$ satisfy the trace equation

$$
|\lambda(2)|=|\lambda(1)|+|\lambda(3)|
$$

together with

$$
\sum_{j \in I_{2}} \lambda_{j}(2) \leqslant \sum_{j \in I_{1}} \lambda_{j}(1)+\sum_{j \in I_{3}} \lambda_{j}(3)
$$

for every triple $\left(I_{1}, I_{2}, I_{3}\right)$ of subsets of $\{1, \ldots, n\}$ of the same cardinality $r<n$ and $c_{\lambda\left(I_{1}\right), \lambda\left(I_{3}\right)}^{\lambda\left(I_{2}\right)} \neq 0$;

3. if $\lambda_{j}(i)$ is an integer for each $1 \leqslant j \leqslant n, i \in\{1,2,3\}$, (1) and (2) are equivalent to $c_{\lambda(1), \lambda(3)}^{\lambda(2)} \neq 0$.

T. Klein [Kle68] noted the equivalence of (3) with short exact sequences of finite abelian $p$-groups, while A. Klyachko [Kly98] proved the equivalence of (1) and (2). The equivalence of (1) and (3) immediately proves Horn's Conjecture.

Corollary 1.3. Theorem 1.2 implies Horn's Conjecture.

While Horn's original conjecture was stated differently, this theorem is commonly referred to now as Horn's conjecture. 
In order to rewrite Theorem 1.2 in a more convenient form, denote $\mathcal{K}(n, 3)$ as the set of all triples $(\lambda(1), \lambda(2), \lambda(3))$ of weakly decreasing sequences of $n$ real numbers for which there exist $n \times n$ complex Hermitian matrices, and denote the set of all triples $(\lambda(1), \lambda(2), \lambda(3))$ of weakly decreasing sequences of $n$ integers such that $c_{\lambda(1), \lambda(3)}^{\lambda(2)} \neq 0$ as $\mathcal{L} \mathcal{R}(n, 3)$.

Theorem 1.4. With the above notation, the set $\mathcal{K}(n, 3)$ is a rational convex polyhedral cone of dimension $3 n-3$ defined by the trace identity and the Horn-type inequalities (1.1); this is called the Klyachko's cone. Moreover, $\mathcal{L} \mathcal{R}(n, 3)=\mathcal{K}(n, 3) \cap \mathbb{Z}^{3 n}$.

A similar polytopal description for the set of possible triples of finite abelian $p$ groups defined such partitions is described in [Kle68]. As mentioned above, not all of the Horn-type inequalities are independent. A complete and minimal list was proved by A. Knutson, T. Tao, and C. Woodward.

Theorem 1.5 ([KTW04]). Klyachko's cone $\mathcal{K}(n, 3)$ is defined by triples $(\lambda(1), \lambda(2), \lambda(3))$ of weakly decreasing sequences of $n$ real numbers that satisfy the trace equation

$$
\lambda(2)=\lambda(1)+\lambda(3)
$$

and the homogeneous linear inequalities

$$
\sum_{j \in I_{2}} \lambda_{j}(2) \leqslant \sum_{j \in I_{1}} \lambda_{j}(1)+\sum_{j \in I_{3}} \lambda_{j}(3)
$$

for every triple $\left(I_{1}, I_{2}, I_{3}\right)$ of subsets of $\{1, \ldots, n\}$ of the same cardinality $r<n$ for which $c_{\lambda\left(I_{1}\right), \lambda\left(I_{3}\right)}^{\lambda\left(I_{2}\right)}=1$. Furthermore, this is a minimal defining list. 


\subsubsection{Stretched polynomials and Geometric Complexity The- ory}

The stretched function $P(N)=c_{N \lambda, N \mu}^{N \nu}$ for $N \in \mathbb{Z}_{\geqslant 0}$ for fixed partitions $\lambda, \mu, \nu$ has interesting combinatorial properties and has been studied by many people (see, for instance, [KTT04], [KTT06a], and [KTT06b]). Inspired by A.N. Kirillov's proof that stretched Kostka numbers are polynomial in the stretching factor $N$ for fixed partitions, R. King, C. Tollu, and F. Toumazet made a similar conjecture for stretched Littlewood-Richardson numbers and summarized related existing conjectures in [KTT04]. Some of them are:

1. (Polynomiality Conjecture) $P$ is a polynomial with nonnegative rational coefficients.

2. (Saturation Conjecture) If $P(1)=0$, then $P(N)=0$ for all $N \geqslant 1$.

3. (Fulton's Conjecture) If $P(1)=1$, then $P(N)=1$ for all $N \geqslant 1$.

4. (KTT Conjecture I) If $P(1)=2$, then $P(N)=N+1$ for all $N \geqslant 1$.

5. (KTT Conjecture II) If $P(1)=3$, then $P(N)=2 N+1$ or $P(N)=\left(\begin{array}{c}N+2 \\ N\end{array}\right)$ for all $N \geqslant 1$

The stretched polynomial has been explicitly calculated in some cases when $P(1)>3$, but as $P(1)$ increases so it seems does the number of possible forms $P(N)$ can take.

While no proof exists yet for the coefficients of $P$ being nonnegative rational coefficients, H. Derksen and J. Weyman [DW02] proved that $P$ is a polynomial and E. Rassart [Ras04] proved this again shortly afterwards, while Fulton's Conjecture was first proven combinatorially by A. Knutson, T. Tao, and C. Woodward [KTW04], then later geometrically by P. Belkale [Bel07], and again using quivers [DW11]. The 
KTT Conjecture I was first proven combinatorially by C. Ikenmeyer [Ike16] and then geometrically and by using quivers by C. Sherman in [She15] and [She17], respectively. The KTT Conjecture II remains to be proved.

This stretched polynomial arises naturally in the study of Littlewood-Richardson coefficients because it is the Ehrhart polynomial of Knutson and Tao's hive models, that is, the polynomial $P$ associated to a polytope $T$ such that the number of lattice points in the scaled polytope $N T$ is precisely $P(N)$. Such a function allows an interesting interplay between enumerative combinatorics and convex geometry. In particular, these stretched polynomials are used in Geometric Complexity Theory (GCT).

GCT was introduced by K. Mulmuley and M. Sohoni in a series of papers (see [MS07], [MS01a], [MS01b], [MS08], [MNS12], [MS17], [Mul10], [Mul11]) in the early 2000's with the purpose of approaching fundamental problems in complexity theory, such as P vs. NP, through algebraic geometry and representation theory. Previously, [KT01] and [LM06] had independently shown that the positivity of LittlewoodRichardson coefficients could be computed in polynomial time while [Nar05] had shown that the actual computation of these numbers was a \#P-complete problem, the complexity class for problems for which (unless $\mathrm{P}=\mathrm{NP}$ ) there does not exist a polynomial time algorithm for computing them (rather, it takes an exponential time in the worst case), and such that the computation is at least as difficult as every $\mathrm{P}$ problem.

The following is the main theorem of [MS05], where the bit length of a partition $\lambda=\left(\lambda_{1}, \ldots, \lambda_{n}\right), \lambda_{n}>0$, is the bit length of the specifications: $\sum_{i=1}^{k} n \log _{2} \lambda_{i}$. 
Theorem 1.6. Deciding whether $c_{\lambda, \mu}^{\nu}$ is positive can be computed in strongly polynomial time in the sense of [GLS93]. This means that the number of arithmetic steps is polynomial in the number of positive parts of $\nu$ (say $n$ ), does not depend on the bit lengths of $\lambda_{i}, \mu_{j}, \nu_{k}$, and the bit length of every intermediate operand that arises in the algorithm is polynomial in the total bit length of $\lambda, \mu, \nu$.

In fact, by attaching zeros to the partitions, one can subsume the dependence on $n$ into the bit lengths of $\lambda, \mu$, and $\nu$. This is especially amazing as the the dimension of the Schur module $S^{\nu}(V)$ is exponential in $n$ and the bit lengths of the $\nu_{k}$ 's, yet deciding if an exponential dimensional object $S^{\nu}(V)$ arises in the decomposition of another exponential dimensional object $S^{\lambda}(V) \otimes S^{\mu}(V)$ can be decided in time that is polynomial in only $n$ and the bit lengths of the labels $\lambda, \mu$, and $\nu$.

Because of results such as these along with the ubiquity of the plethysm problem and related problems in representation theory, GCT allows one to compare the complexity of several problems. The proof of deciding the positivity of a LittlewoodRichardson coefficient relies on two main points: a polyhedral interpretation of these numbers and the saturation theorem. While we define a polytope for the generalized Littlewood-Richardson coefficients to prove a similar result (Theorem 4.13), it would be nice to have a purely combinatorial algorithm, such as those of max-flow or weighted matching problems in combinatorial optimization. Much work has been made towards this for single Littlewood-Richardson coefficients (see [BI09], [BI13], and [Ike16]). 


\subsubsection{Branching rules}

Littlewood-Richardson coefficients appear in many contexts in representation theory and algebraic combinatorics, such as the coefficients in the decomposition of a product of symmetric polynomials or the tensor product of irreducible representations of GL $(n)$. Similarly, sums of products of these coefficients, which we call generalized Littlewood-Richardson coefficients ${ }^{1}$ throughout this paper, appear naturally in the decompositions of various algebraic objects. In particular, two of the generalized Littlewood-Richardson coefficients that we study describe the multiplicities in the branching rules of restricted representations of GL(n), as described by [HJ09] and [HTW05]. In general, a representation $(\rho, V)$ of a group $G$ naturally restricts to a representation $\left(\left.\rho\right|_{H}, V\right)$ of a subgroup $H \leqslant G$. If $V$ is an irreducible representation of $G$, then $V$ does not necessarily remain irreducible when considered as a representation of $H$. Branching rules describe how the restricted representation decomposes into a sum of irreducibles, thus allowing an understanding of the representation theory of all subgroups of a given group from the representation theory of the group itself. By Frobenius reciprocity, the number of times an irreducible representation $W$ of $H$ occurs in the restriction of an irreducible representation $V$ of $G$ is the same as the number of times $V$ occurs in the induced representation of $W$ to $G$, so alternatively one may understand the representation of a group from the representation theory of all of its proper subgroups. Because of this importance, branching rules are a vital and classical study of representation theory, where a systematic modern interpretation in

\footnotetext{
${ }^{1}$ The Littlewood-Richardson coefficients which we described above are known as type $A$ because they arise as the structure constants for GL $(n)$ rather than other algebraic groups. It's common in the literature to call the structure constants for other algebraic groups (e.g. , $\mathrm{O}(n), \operatorname{Sp}(2 n)$, etc.) generalized Littlewood-Richardson coefficients, though we never mean this when using the term in this thesis.
} 
terms of dual pairs was given by R. Howe [How95].

While there is no known way to describe the multiplicities for all of the branching rules of the restricted representations of $\mathrm{GL}(n)$ using quiver theory, we show that we can do exactly such for two of them, that is, we describe each of these coefficients as the dimension of a weight space of semi-invariants for a certain quiver, dimension vector, and weight. More generally, we do this for the generalized Littlewood-Richardson coefficients

$$
\begin{aligned}
& f_{1}(\lambda(1), \ldots, \lambda(m)):=\sum c_{\alpha(1), \alpha(2)}^{\lambda(1)} c_{\alpha(2), \alpha(3)}^{\lambda(2)} \cdots c_{\alpha(m-1), \alpha(m)}^{\lambda(m-1)} c_{\alpha(m), \alpha(1)}^{\lambda(m)} m=2 k, k \geqslant 2 \\
& f_{2}(\lambda(1), \ldots, \lambda(m)):=\sum c_{\lambda(1), \lambda(2)}^{\alpha(1)} c_{\alpha(1), \alpha(2)}^{\lambda(3)} \cdots c_{\alpha(m-4), \alpha(m-3)}^{\lambda(m-2)} c_{\lambda(m-1), \lambda(m)}^{\alpha(m-3)} \quad m \geqslant 3
\end{aligned}
$$

where the summation in each ranges over all partitions $\alpha(i)$. The first multiplicity describes the coefficients arising from the branching rule for the diagonal embedding $\mathrm{GL}(n) \subseteq \mathrm{GL}(n) \times \mathrm{GL}(n)$ in the case $m=6$ while the second describes the branching rule for the direct sum embedding $\operatorname{GL}(n) \times \mathrm{GL}\left(n^{\prime}\right) \subseteq \mathrm{GL}\left(n+n^{\prime}\right)$ when $m=6$, and $f_{2}(\lambda(1), \lambda(2), \lambda(3)):=c_{\lambda(1), \lambda(3)}^{\lambda(2)}$. In addition, we study

$$
f_{3}(\lambda(1), \ldots, \lambda(m)):=\sum c_{\lambda(1), \alpha(1)}^{\lambda(2)} c_{\alpha(1), \alpha(2)}^{\lambda(3)} \cdots c_{\alpha(m-4), \alpha(m-3)}^{\lambda(m-2)} c_{\alpha(m-3), \lambda(m)}^{\lambda(m-1)}, \quad m \geqslant 3
$$

where again, the sum ranges over all partitions $\alpha(i)$ and $f_{3}(\lambda(1), \lambda(2), \lambda(3)):=$ $c_{\lambda(1), \lambda(3)}^{\lambda(2)}$

The multiplicity (1.2) for the branching rule of the diagonal embedding of GL( $n$ ) was first proved in [Kin71], while the multiplicity (1.3) for the branching rule of the direct sum embedding was first proven in [Kin70]; both are also proved in [HTW05] (see also [HJ09] and [Koi89] for further discussion). The multiplicity (1.4) instead describes the tensor product multiplicities for extremal weight crystals of type $A_{+\infty}$, 
using a combinatorial rule found by Kashiwara [Kas90] similar to the LittlewoodRichardson rule that described the irreducible components of the tensor product of irreducible representations of the quantized universal enveloping algebra of a symmetrizable Kac-Moody algebra $\mathfrak{g}$ as described in [Kwo09] (see also [Kwo10]) again when $m=6$. This generalized multiplicity is studied in the context of quiver theory in [Chi08] and [Chi09], and is found to have connections with long exact sequences of finite, abelian p-groups, parabolic affine Kazhdan-Lusztig polynomials, and decomposition numbers for $q$-Schur algebras.

Recently, Littlewood-Richardson coefficients have been of vital interest in geometric complexity theory which seeks to determine the complexity of computational problems by using tools from algebraic geometry and representation theory to provide lower bounds, and the complexity of the calculations is quite commonly compared to that of computing certain multiplicities like Littlewood-Richardson coefficients and Kronecker coefficients. Understanding the complexity of certain cases of these generalized coefficients or even whether they're nonzero can then be used to compare the difficulty of other computational problems. The method of determining the complexity relies on a common technique in combinatorics, namely, associating a polytope to the multiplicity in such a way that the number of lattice points of the polytope is precisely this number. Because the polytope is defined by a system of linear inequalities, combinatorial optimization may then be used to determine the complexity of the multiplicities as well as the properties of the polytope.

A. Knutson and T. Tao [KT99] provided a polytopal description of LittlewoodRichardson coefficients, allowing them to give a combinatorial proof of the saturation 
of the coefficients (Theorem 1.1) and complete the proof of Horn's conjecture (Theorem 1.2). Derksen and Weyman [DW00a] then reproved the saturation property in the context of quiver representations by using the saturation of weight spaces of semi-invariants. The motivation for this thesis may then be summarized as providing an explicit quiver theoretic interpretation of these generalized Littlewood-Richardson coefficients in order to prove their saturation and use results of quiver theory to study their combinatorial and geometric properties.

\subsection{Main results}

One of the main and most useful results is that of the saturation of these multiplicities.

Theorem 1.7. Let $\lambda(1), \ldots, \lambda(m)$ be weakly decreasing sequences of $n$ integers for $m \geqslant 3$. For every integer $r \geqslant 1$,

1. $f_{1}(r \lambda(1), \ldots, r \lambda(m)) \neq 0 \Longleftrightarrow f_{1}(\lambda(1), \ldots, \lambda(m)) \neq 0 \quad m \geqslant 4$ and even,

2. $f_{2}(r \lambda(1), \ldots, r \lambda(m)) \neq 0 \Longleftrightarrow f_{2}(\lambda(1), \ldots, \lambda(m)) \neq 0$.

The saturation of multiplicity (1.4) is Theorem 1.4 in [Chi08].

We extend the results of [KT99] by using their hive models to provide a polytopal description of the generalized Littlewood-Richardson coefficients in Section 4.3. By using results in combinatorial optimization theory and the above saturation property, we prove the following theorem.

Theorem 1.8. Determining whether the multiplicities (1.2), (1.3), and (1.4) are positive or not can be decided in polynomial time. Even more, they can be decided in strongly polynomial time in the sense of [Tar86].

Recall that Horn's conjecture (Theorem 1.2) relates the set of possible eigenval- 
ues arising from a sum of Hermitian matrices to the nonvanishing of LittlewoodRichardson coefficients. To describe a corresponding statement of the conjecture to generalized Littlewood-Richardson coefficients, we need to define some notation. For an $m$-tuple $\left(I_{1}, \ldots, I_{m}\right)$ of subsets of $\{1, \ldots, n\}$, define the following weakly decreasing sequences of integers (recall the notation defined prior to Theorem 1.1):

$$
\underline{\lambda}_{1}\left(I_{i}\right)= \begin{cases}\lambda^{\prime}\left(I_{i}\right) & i \text { even } \\ \lambda^{\prime}\left(I_{i}\right)-\left(\left(\left|I_{i}\right|-\left|I_{i-1}\right|-\left|I_{i+1}\right|\right)^{n-\left|I_{i}\right|}\right) & i \text { odd }\end{cases}
$$

where we identify $I_{0}$ and $I_{m}$. Define the set $\mathcal{K}_{1}(n, m) \subseteq \mathbb{R}^{m n}, m \geqslant 4$ and even, to be all $m$-tuples $(\lambda(1), \ldots, \lambda(m))$ of weakly decreasing sequences of $n$ reals that satisfy $\sum_{i \text { even }}|\lambda(i)|=\sum_{i \text { odd }}|\lambda(i)|$ and

$$
\sum_{j \in I_{i}} \sum_{i \text { even }} \lambda(i)_{j} \leqslant \sum_{j \in I_{i}} \sum_{i \text { odd }} \lambda(i)_{j}
$$

for every tuple $\left(I_{1}, \ldots, I_{m}\right)$ such that $\underline{\lambda}\left(I_{i}\right), 1 \leqslant i \leqslant m$, are partitions and

$$
f_{1}\left(\underline{\lambda}_{1}\left(I_{1}\right), \ldots, \underline{\lambda}_{1}\left(I_{m}\right)\right) \neq 0
$$

This makes $\mathcal{K}_{1}(n, m)$ a rational convex polyhedral cone in $\mathbb{R}^{m n}$, which we call the generalized Klyachko's cone. A corresponding statement of Horn's conjecture for this multiplicity is then as follows, where we describe the generalized eigenvalue problem for $f_{1}$ in Section 3.4 .

Theorem 1.9. The following statements are true.

1. The cone $\mathcal{K}_{1}(n, m) \subseteq \mathbb{R}^{m n}, m \geqslant 4$ and even, consists of all $m$-tuples $(\lambda(1), \ldots, \lambda(m))$ of weakly decreasing sequences of $n$ reals satisfying $\sum_{i \text { even }}|\lambda(i)|=\sum_{i \text { odd }}|\lambda(i)|$ and

$$
\sum_{j \in I_{i}} \sum_{i} \lambda(i)_{j} \leqslant \sum_{j \in I_{i}} \sum_{i} \lambda(i)_{j}
$$


for every tuple $\left(I_{1}, \ldots, I_{m}\right)$ such that the $\underline{\lambda}_{1}\left(I_{i}\right), 1 \leqslant i \leqslant m$, are partitions and

$$
f_{1}\left(\underline{\lambda}_{1}\left(I_{1}\right), \ldots, \underline{\lambda}_{1}\left(I_{m}\right)\right)=1 .
$$

2. If $(\lambda(1), \ldots, \lambda(m)) \in \mathcal{K}_{1}(n, m)$, then the tuple satisfies the generalized eigenvalue problem for $f_{1}$.

3. If $\lambda(1), \ldots, \lambda(m)$ are weakly decreasing sequences of $n$-integers, then

$$
(\lambda(1), \ldots, \lambda(m)) \in \mathcal{K}_{1}(n, m) \Longleftrightarrow f_{1}(\lambda(1), \ldots, \lambda(m)) \neq 0 .
$$

4. $\operatorname{dim} \mathcal{K}_{1}(n, m)=m n-1$.

In particular, this provides a recursive procedure for finding all nonzero generalized Littlewood-Richardson coefficients of this type. We use this description to describe all facets of the cone of effective weights in the case $n=2, m=6$, and find the minimal set of inequalities on the $\lambda(i)$ (see Example 3.21 and the Appendix).

We similarly describe the generalization of Horn's conjecture for the multiplicity $f_{2}$ in Theorem 3.29 and Proposition 3.30 after defining the corresponding generalized Klyachko's cone $\mathcal{K}_{2}(n, m)$. While the statement is slightly complicated by the parity of $m$ affecting the conditions, the advantage of $f_{2}$ is that a tuple lies in $\mathcal{K}_{2}(n, m)$ if and only if it satisfies the generalized eigenvalue problem. In either case, we will show that $\mathcal{K}_{2}(n, m)$ is a rational convex polyhedral cone of dimension $m n-1$. A similar description of $\mathcal{K}_{3}(n, m)$ for $f_{3}$ is provided in [Chi08], where it is shown that $\operatorname{dim} \mathcal{K}_{3}(n, m)=m n-1$. We recall the description of $\mathcal{K}_{3}(n, m)$ in Section 4.1.

One consequence of the descriptions of the sequences in the cones $\mathcal{K}_{i}(n, m), i \epsilon$ $\{1,2,3\}$, is the following factorization formula. 
Theorem 1.10. Let $k \in\{1,2,3\}$ and $(\lambda(1), \ldots, \lambda(m)) \in \mathcal{K}_{k}(n, m) \cap \mathbb{Z}^{m n-1}$. We have the factorization

$$
f_{k}(\lambda(1), \ldots, \lambda(m))=f_{k}\left(\lambda(1)^{*}, \ldots, \lambda(m)^{*}\right) \cdot f_{k}\left(\lambda(1)^{\#}, \ldots, \lambda(m)^{\#}\right)
$$

where $m \geqslant 3, m$ is even if $k=1$, and

$$
\begin{gathered}
\lambda(p)^{*}=\left(\lambda(p)_{i_{j_{1}}}, \ldots, \lambda(p)_{i_{j_{r}}}\right), \quad I_{j}=\left\{i_{j_{1}}, \ldots, i_{j_{r}}\right\}, \\
\lambda(p)^{\#}=\left(\lambda(p)_{\tilde{i}_{j_{1}}}, \ldots, \lambda(p)_{\tilde{i}_{j_{n-r}}}\right), \quad S \backslash I_{j}=\left(\tilde{i}_{j_{1}}, \ldots, \tilde{i}_{j_{n-r}}\right) .
\end{gathered}
$$

We also investigate the stretched Littlewood-Richardson polynomials $f_{i}(N \lambda(1), \ldots, N \lambda(m))$ for certain $m$-tuples $(\lambda(1), \ldots, \lambda(m))$ in Section 4.2. The tu-

ples we investigate produce the same behavior for the stretched polynomials as the stretched Littlewood-Richardson polynomials for a single coefficient, namely, they satisfy the conjectures stated in Subsection 1.1.2, providing evidence that the conjectures for the stretched polynomials for a single Littlewood-Richardson coefficient extend to those of generalized Littlewood-Richardson coefficients.

\subsection{Organization of the thesis}

The organization of this paper is as follows. In Chapter 2 we provide background on quiver invariant theory and state a certain saturation property for effective weights of quivers proven by H. Derksen and J. Weyman [DW00a] as well as a detailed description of the facets of the cone of effective weights. The quivers associated to multiplicities (1.2) and (1.3) are defined in Chapter 3 and their saturation properties are proved there. We describe the facets of these quivers in Section 3.2, which then allows a description of the Horn-type inequalities of the multiplicities and the generalized eigenvalue problems for these multiplicities in the remainder of the chapter. In 
Chapter 4, we use these results to further study the combinatorics and complexity of these numbers by extending results for single Littlewood-Richardson coefficients. We first prove factorization formulas for these generalized numbers and then explicitly calculate the stretched Littlewood-Richardson polynomials in certain cases, verifying that the numbers in these cases satisfy the same conjectures as those for single Littlewood-Richardson coefficients. We finally provide a polytopal description of the multiplicities in Section 4.3 and prove the complexity of computing their positivity. 


\section{Chapter 2}

\section{Background material}

We review in this chapter the representation theory, complexity theory, and quiver theory that we will use throughout the remainder of this thesis. We first recall the construction of the irreducible representations of GL $(n)$ before discussing Knutson and Tao's hive models, which can be used to show that the positivity of LittlewoodRichardson coefficients can be computed in strongly polynomial time. The rest of this chapter rephrases the representation theory in the context of quiver invariant theory.

\subsection{Representation theory of GL $(n)$}

Throughout this section $V$ is complex vector space of dimension $n$. The general linear group $\mathrm{GL}(V)$ is the set of all invertible endomorphisms of $V$ and carries the structure of an algebraic group, meaning that $\mathrm{GL}(V)$ is an algebraic variety and the multiplication and inverse operations are morphisms of algebraic varieties. The affine coordinate ring of GL( $V)$ is

$$
\mathbb{C}[\mathrm{GL}(V)]=\mathbb{C}\left[\operatorname{End}_{\mathbb{C}}(V)\right]\left[\frac{1}{\operatorname{det}}\right]
$$

where $\operatorname{det} \epsilon \operatorname{End}_{\mathbb{C}}(V)$ is the determinant function. If $V$ has a specified basis, $\operatorname{End}_{\mathbb{C}}(V) \cong$ $\operatorname{Mat}_{n}(\mathbb{C})$, the space of $n \times n$ matrices, and so $\mathbb{C}[\mathrm{GL}(V)] \cong \mathbb{C}\left[x_{11}, x_{12}, \ldots, x_{n n}\right]\left[\frac{1}{\mathrm{det}}\right]$. 
Definition 2.1. A linear representation of $\mathrm{GL}(V)$ is a finite-dimensional complex vector space $W$ and a group homomorphism $\rho: \mathrm{GL}(V) \rightarrow \mathrm{GL}(W)$. We say that

1. $\rho$ is a rational representation of $\mathrm{GL}(V)$ if the matrix entries of $\rho(g)=$ $\left(\rho_{i j}(g)\right)$ with respect to some (equivalently, any) basis belong to $\mathbb{C}[\mathrm{GL}(V)]$.

2. $\rho$ is a polynomial representation of $\mathrm{GL}(V)$ if the matrix entries belong to the polynomial algebra $\mathbb{C}\left[\operatorname{End}_{\mathbb{C}}(V)\right]$.

Example 2.2. 1. A standard example of a polynomial representation is the 1dimensional representation $\operatorname{det}_{V}^{r}: \mathrm{GL}(V) \rightarrow \mathrm{GL}(\mathbb{C})$ for any $r \in \mathbb{Z}$, defined by

$$
\operatorname{det}_{V}^{r}(g)(x):=\operatorname{det}(g)^{r} \cdot x \quad \forall x \in \mathbb{C}, \forall g \in \mathrm{GL}(V)
$$

When $r=0$ this is simply the trivial representation, while for $r=1$ this is the determinant representation. We then call $\operatorname{det}_{V}^{r}$ is the $r^{\text {th }}$ power of the determinant representation.

2. Because a representation $(\rho, V)$ of a group $G$ defines a $k[G]$-module, where $k[G]$ is the group algebra, via the action $g \cdot v:=\rho(g) v$, and conversely a $k[G]$-module $V$ similarly defines a representation, we may identify representations with modules. Thus, any construction on vector spaces or modules yields new representations. For instance, the representation $(\rho, V)$ defines the dual representation $\left(\rho^{-t}, V^{*}\right)$, where $V^{*}$ is the dual vector space and $\rho^{-t}$ is the inverse transpose of $\rho$ (this is the defining homomorphism in order to respect the natural pairing between $V$ and $V^{*}$ ). Similarly, for any $r \in \mathbb{Z}_{\geqslant 0}$, we can define the $r^{t h}$ exterior and symmetric power representations $\left(\rho^{\otimes r}, \wedge^{r} V\right)$ and $\left(\rho^{\otimes r}, \operatorname{Sym}^{r} V\right)$.

The fundamental goal of representation theory is to classify all representations up to isomorphism. Because the base field is $\mathbb{C}$, the group $\mathrm{GL}(n)$ is linearly reductive, 
meaning the conclusion of Maschke's Theorem holds and thus every locally finitedimensional rational representation decomposes in a unique way into a direct sum of irreducible representations. Because of this, the fundamental goal is equivalent to classifying all irreducible representations up to isomorphism. In the remainder of this section we review this classification theory for $\mathrm{GL}(n)$ and related results we will need later.

\subsubsection{Schur modules}

A partition $\lambda=\left(\lambda_{1}, \ldots, \lambda_{r}\right)$ of length $r$ is a weakly decreasing sequence $\lambda_{1} \geqslant \ldots \geqslant$ $\lambda_{r} \geqslant 1$ of positive integers. We identify $\lambda=\left(\lambda_{1}, \ldots, \lambda_{r}\right)$ with $\left(\lambda_{1}, \ldots, \lambda_{r}, 0, \ldots, 0\right)$, and denote the set of all partitions of length at most $r$ as $\mathcal{P}_{r}$. In particular, $\mathcal{P}_{r}$ forms a semigroup.

To each partition $\lambda$, we associate a Young diagram, which consists of left justified rows, where row $i$ has $\lambda_{i}$ boxes $^{1}$. The conjugate partition, denoted $\lambda^{\prime}$, is attained by reflecting the diagram over the main diagonal. That is, we switch the rows and columns.

Example 2.3. The partition $\lambda=(5,3,2,1)$ has conjugate partition $\lambda^{\prime}=(4,3,2,1,1)$, and the corresponding Young diagrams are
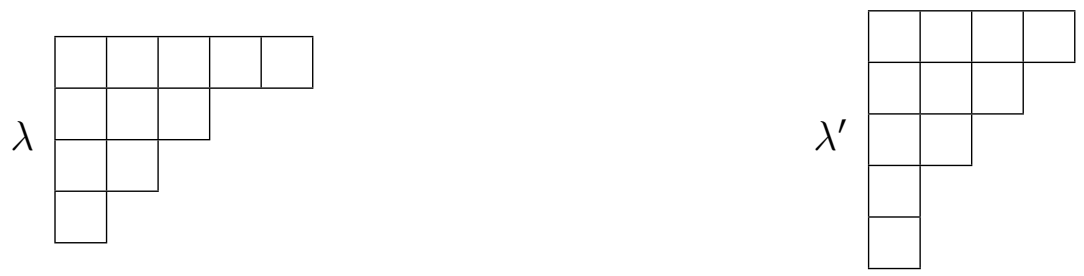

Though seemingly simple, Young diagrams provide an essential way of organizing and manipulating the combinatorial data used in much of representation theory. For

\footnotetext{
${ }^{1}$ Though this is the general convention, the Francophone version is flipped upside down.
} 
instance, besides being used below to construct the irreducible representations of $\mathrm{GL}(n)$ and $\mathrm{SL}(n)$, they are used to construct the irreducible representations of the symmetric group $S_{n}$ [Sag01], to study symmetric polynomials [Mac15], are essential in Schubert calculus [Ful98], and appear in many other places in mathematics.

Naturally, we want to fill the boxes with some algebraic data. We follow [Ful97a] to construct the irreducible representations of $\operatorname{GL}(n)$. Given a vector space $V$ of dimension $n$, denote $V^{\lambda}=V^{|\lambda|}$ as the set of all functions (or fillings) $T$ from the set of boxes of the Young diagram of $\lambda$ to $V$. That is, a filling places a vector $v_{i} \in V$ into a box in the Young diagram of $\lambda$ and all boxes are filled in this way. An element $T \in V^{\lambda}$ is called a Young tableau. For instance, if $\lambda=(5,3,2,1)$, then one filling is

\begin{tabular}{|l|l|l|l|l|}
\hline$v_{8}$ & $v_{2}$ & $v_{3}$ & $v_{6}$ & $v_{11}$ \\
\hline$v_{1}$ & $v_{9}$ & $v_{7}$ & \multicolumn{2}{|c}{} \\
\cline { 1 - 2 }$v_{10}$ & $v_{5}$ & \multicolumn{2}{|c}{} \\
\cline { 1 - 2 }$v_{4}$ & \multicolumn{2}{|c}{} \\
\cline { 1 - 2 }
\end{tabular}

Because it is tedious and notationally cumbersome to write $v_{i}$ as $i$ ranges over $\{1, \ldots, n\}$, we adopt the convention for now on only to write $i$ in a box. To define the irreducible representations, we will want to define a certain equivalence relation on the set of fillings.

Definition 2.4. An exchange of $T \in V^{\lambda}$ is a swap of a subset of elements in column $j$ with a subset of elements in column $i$ which are the same in number and preserve the order of the entries in each column, listed from top to bottom.

Example 2.5. For the partition $\lambda=(5,3,2,1)$, the following is an example of an 
exchange of the above filling:

\begin{tabular}{|c|c|c|c|c|c|c|c|}
\hline 8 & 2 & 3 & \begin{tabular}{|l|l|}
6 & 11
\end{tabular} & 2 & 8 & 3 & \begin{tabular}{l|l}
6 & 11
\end{tabular} \\
\hline 1 & 9 & 7 & \multirow{3}{*}{$\sim$} & 1 & 10 & 7 & \\
\hline 10 & 5 & & & 9 & 4 & & \\
\hline 4 & & & & 5 & & & \\
\hline
\end{tabular}

However, the following swap fails to be an exchange because it does not preserve the original order of the elements in each column:

\begin{tabular}{|c|c|c|c|c|c|c|c|}
\hline 8 & 2 & 3 & \begin{tabular}{l|l}
6 & 11
\end{tabular} & 9 & 8 & 3 & \begin{tabular}{l|l}
6 & 11 \\
\end{tabular} \\
\hline 1 & 9 & 7 & \multirow{3}{*}{$\psi$} & 1 & 10 & 7 & \\
\hline 10 & 5 & & & 2 & 4 & & \\
\hline 4 & & & & 5 & & & \\
\hline
\end{tabular}

Let $\lambda=\left(\lambda_{1}, \ldots, \lambda_{r}\right)$ be a partition and $\mu=\lambda^{\prime}=\left(\mu_{1}, \ldots, \mu_{s}\right)$ the conjugate partition. Define a map $\Lambda: V^{\lambda} \rightarrow \wedge^{\mu_{1}} V \otimes \cdots \otimes \bigwedge^{\mu_{s}} V$ by taking the tensor products of the exterior products of the column entries. (Recall that although we are labeling the entries of the Young diagram by numbers, these denote elements of the vector space $V$.)

We define the Schur module $S^{\lambda} V$ to be $\otimes_{i=1}^{s} \wedge^{\mu_{i}} V / Q^{\lambda} V$, where $Q^{\lambda} V$ is the subspace spanned by all exchanges. That is, the space $\wedge T-\sum_{S} \lambda(S)$, where the sum ranges over all $S \in V^{\lambda}$ obtained from a tableau $T$ by exchanging two given columns and a given subset in the right column.

Example 2.6. 1. Let $\lambda=(e)$ for some $e \in \mathbb{Z}_{\geqslant 0}$. Then $\mu=\left(1^{e}\right)$ and $S^{(e)} V=$ $\otimes_{e} \wedge^{1} V / Q^{(e)} V$. Because

$$
Q^{(e)} V=\left\langle v_{1} \otimes \cdots \otimes v_{i} \otimes \cdots v_{j} \otimes \cdots \otimes v_{e}-v_{1} \otimes \cdots \otimes v_{j} \otimes \cdots v_{i} \otimes \cdots \otimes v_{e} \mid 1 \leqslant i<j \leqslant n\right\rangle,
$$

we see that this Schur module is isomorphic to the $e^{\text {th }}$ symmetric power of $V$, $\operatorname{Sym}^{e} V$.

\footnotetext{
${ }^{2}$ The literature commonly uses "Schur" and "Weyl" module interchangeably. While the two have different constructions, the two notions are dual to each other in characteristic zero, which is the only case we're interested in. There are additional methods of construction of Schur modules as well, such as via Schur-Weyl duality and Young symmetrizers.
} 
2. Let $\lambda=\left(1^{e}\right)$. Then $\mu=(e)$ and $S^{\left(1^{e}\right)} V=\Lambda^{(e)} V / Q^{\left(1^{e}\right)} V$, where $Q^{\left(1^{e}\right)} V$ is trivial. Thus, $S^{\left(1^{e}\right)} V$ is isomorphic to the $e^{\text {th }}$ exterior power, $\wedge^{e} V$. This and the previous example show how the Schur modules $S^{\lambda} V$ are generalizations of the natural irreducible representations $\operatorname{Sym}^{e} V$ and $\wedge^{e} V$.

3. If $\lambda$ has at least $n+1>\operatorname{dim} V$ parts, then $\mu_{1} \geqslant n+1$, so $S^{\lambda} V=0$.

Definition 2.7. Let $\lambda$ be a partition. A semistandard Young tableau of shape $\lambda$ is a filling such that the entries in the rows are weakly increasing from left to right and the entries in the columns are strictly increasing from top to bottom with positive integers. The tableau is called standard if no entry occurs more than once.

Example 2.8. Consider an ordered basis $\left\{v_{1}, \ldots, v_{15}\right\}$ of a vector space $V$. As per convention, identify the vector $v_{i}$ with $i$. An example of a semistandard Young tableau of shape $\lambda=(5,3,2,1)$ and a standard one would, respectively, be

\begin{tabular}{|l|l|l|l|l|}
\hline 1 & 3 & 8 & 8 & 8 \\
\cline { 1 - 2 } 4 & 4 & 9 & \multicolumn{2}{|c}{} \\
\cline { 1 - 2 } 6 & 7 & \multicolumn{2}{|c}{} \\
\cline { 1 - 2 } 8 & \multicolumn{3}{|c}{} & \multicolumn{2}{|c}{} \\
\cline { 1 - 2 } & & &
\end{tabular}

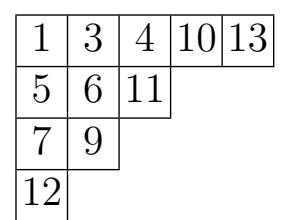

Note that if $|\lambda|>\operatorname{dim} V$, then two distinct rows must contain some of the same entries, and so no semistandard tableaux of shape $\lambda$ can occur. By definition, $S^{\lambda} V=0$ in this case, implying a connection between the number of semistandard tableaux of shape $\lambda$ and the size of $S^{\lambda} V$.

Fix a basis $\left\{e_{1}, \ldots, e_{n}\right\}$ of $V$. Given a semistandard Young tableau of shape $\lambda$ with entries $\{1, \ldots, n\}$, define $e_{T}=\bigwedge T+Q^{\lambda} V \in S^{\lambda} V$.

Theorem 2.9. The space $S^{\lambda} V$ is a polynomial representation of $\mathrm{GL}(V)$ with basis $\left\{e_{T} \mid T\right.$ is a semistandard Young tableau of shape $\lambda$ with entries $\left.\{1, \ldots, n\}\right\}$. 
Moreover, the representations $S^{\lambda} V$ with $\lambda \in \mathcal{P}_{n}$ form a complete set of non-isomorphic irreducible polynomial representations of $\mathrm{GL}(V)$.

The number of semistandard Young tableaux of shape $\lambda$ is given by the hook-length formula:

$$
|S Y T(\lambda)|=\frac{|\lambda| !}{\prod_{(i, j)} h(i, j)},
$$

where the product is over all boxes $(i, j)$ of the Young diagram and $h(i, j)$ is the hook-length at $(i, j)$, meaning the number of bpxes to the right of and below $(i, j)$, including the box itself. Similarly, the number of semistandard Young tableaux of shape $\lambda$ is given by the hook-content formula:

$$
|S S Y T(\lambda)|=\prod_{(i, j)} \frac{n+i-j}{h(i, j)},
$$

where the boxes are filled with entries from the set $\{1, \ldots, n\}$. Semistandard Young tableaux are also in bijection with Gelfand-Tsetlin patterns, which is the basis of the content of Subsection 2.1.2.

We note that if $\lambda=\left(\lambda_{1}, \ldots, \lambda_{n}\right) \in \mathcal{P}_{n}$, the Schur function $s_{\lambda}$ is defined by

$$
s_{\lambda}\left(x_{1}, \ldots, x_{n}\right)=\frac{\operatorname{det}\left(x_{i}^{\lambda_{j}+n-j}\right)}{\operatorname{det}\left(x_{i}^{n-j}\right)} .
$$

For $\lambda \in \mathcal{P}_{n}$, the character $\chi_{\lambda}$ of the irreducible polynomial representation $S^{\lambda} V$ is precisely

$$
\chi_{\lambda}(g)=s_{\lambda}\left(x_{1}, \ldots, x_{n}\right),
$$

where $x_{1}, \ldots, x_{n}$ are the eigenvalues of the matrix $g \in \mathrm{GL}(V)$.

One consequence of Theorem 2.9 is that the dimension of $S^{\lambda} V$ is one if and only if there is only one semistandard Young tableau using the numbers $1, \ldots, n$. But this is 
clearly equivalent to $\lambda$ being a rectangle with the row $i$ entries all equaling $i$ and some number of columns $k$. So $\operatorname{dim} S^{\lambda} V=1$ if and only if $\lambda=\left(k^{n}\right)$ for some $k$. Because the determinant representation is of dimension one, we may conclude that in this case $S^{\lambda} V=\operatorname{det}_{V}^{k}$. Here we say that the representation has weight $k$.

For the analogous classification of rational representations, we need to work over all possible integers rather than just positive ones. Let $\Lambda_{n}$ denote the set of all $n$-tuples of weakly decreasing integers:

$$
\Lambda_{n}:=\left\{\left(\lambda_{1}, \ldots, \lambda_{n}\right) \in \mathbb{Z}^{n} \mid \lambda_{1} \geqslant \cdots \geqslant \lambda_{n}\right\} .
$$

This is the set of dominant weights for $\operatorname{GL}(V)$. Suppose $\lambda \in \Lambda_{n}$. If $\lambda_{n}<0$, define $S^{\lambda} V=S^{\gamma} V \otimes \operatorname{det}_{V}^{\lambda_{n}}$, where $\gamma=\left(\lambda_{1}-\lambda_{n}, \ldots, \lambda_{n-1}-\lambda_{n}, 0\right)$, which is a partition ${ }^{3}$. In particular, if $\lambda \in \mathcal{P}_{n}$, then

$$
S^{\lambda} V \cong S^{\gamma} V \otimes \operatorname{det}_{V}^{\lambda_{n}} .
$$

Moreover, if $\lambda, \mu \in \Lambda$, then for $r, s \in \mathbb{Z}$ such that $\lambda_{i}+r=\mu_{i}+s \forall 1 \leqslant i \leqslant n$, there is an isomorphism

$$
S^{\lambda} V \otimes \operatorname{det}_{V}^{r} \cong S^{\mu} V \otimes \operatorname{det}_{V}^{s} .
$$

Theorem 2.10 ([Ful97a], Theorem 8.2). The representations $S^{\lambda} V$ with $\lambda \in \Lambda_{n}$ form a complete set of non-isomorphic irreducible rational representations of GL( $V)$.

Note that if $\rho: \mathrm{GL}(V) \rightarrow \mathrm{GL}(W)$ is a rational representation of $\mathrm{GL}(V)$, then there exists an $N \gg 0$ such that $\rho \otimes \operatorname{det}_{V}^{N}$ is a polynomial representation. In particular, if $\lambda \in \Lambda_{n}$, then $S^{\lambda} V \cong S^{\gamma} V \otimes \operatorname{det}_{V}^{\lambda_{m}}$, where $\gamma=\left(\lambda_{1}-\lambda_{n}, \ldots, \lambda_{n-1}-\lambda_{n}, 0\right)$ is a partition of at most $n-1$ parts.

\footnotetext{
${ }^{3}$ As might be expected, the connection between partitions and Young tableaux can be extended to tuples of (possibly negative) integers and a negative Young tableaux theory [Ste87]. In this way, explicit bases of these representations may be given, though we will not need this theory here.
} 
This shows that all (finite-dimensional linear) representations of GL( $V)$ are rational and can be "twisted" by a power of the determinant representation to obtain a polynomial representation. To apply these results to $\mathrm{SL}(V)$, first notice that every irreducible representation for $\mathrm{GL}(V)$ restricts to an irreducible representation of $\mathrm{SL}(V)$, and every irreducible representation of $\mathrm{SL}(V)$ is such a restriction. Because the determinant representation for $\mathrm{SL}(V)$ is trivial, all $\mathrm{SL}(V)$-representations are in fact polynomial, where $S^{\lambda}(V) \cong S^{\mu} V$ if and only if $\lambda_{i}-\mu_{i}=k$ for some fixed $k \in \mathbb{Z}$ for all $i$ by Equation (2.1).

Theorem 2.11. The representations $S^{\lambda} V$ with $\lambda=\left(\lambda_{1}, \ldots, \lambda_{n-1}, 0\right) \in \mathcal{P}_{n}$ form a complete set of non-isomorphic irreducible rational (equivalently, polynomial) representations of $\mathrm{SL}(V)$. Furthermore, if $\lambda, \mu \in \mathcal{P}_{n}$, then $S^{\lambda} V \cong S^{\mu} V$ as $\mathrm{SL}(V)$ representations if and only if $\lambda_{i}-\mu_{i}=k \in \mathbb{Z}$ for all $i$ for some fixed $k$.

There are a few important properties of these representations we will need in future calculations. For a representation $W$ of $\mathrm{SL}(V), W^{\mathrm{SL}(V)}$ is the space of invariants under the left regular action of $\mathrm{SL}(V)$, that is,

$$
W^{\mathrm{SL}(V)}=\{w \in W \mid g \cdot w=w \forall g \in \mathrm{SL}(V)\} .
$$

The action by $\operatorname{SL}(V)$ lifts to that of $\operatorname{GL}(V)$, however equality is only obtained up to a character of $\mathrm{GL}(V)$, and so we also call this the space of semi-invariants. We review invariants and semi-invariants in Subsection 2.2.2 in the context of quivers.

Proposition 2.12. The following are equivalent for a partition $\lambda$ for a vector space $V$ of dimension $n$ :

1. $\operatorname{dim}\left(S^{\lambda} V\right)^{\mathrm{SL}(V)} \neq 0$; 
2. $\operatorname{dim} S^{\lambda} V=1$;

3. $\lambda=\left(k^{n}\right)$ for some $k \in \mathbb{Z}_{\geqslant 0}$;

4. $S^{\lambda} V$ is spanned by a semi-invariant of weight $k$.

The equivalence of (2), (3), and (4) was mentioned previously as a consequence of Theorem 2.9. One direction of the equivalence of (1) with (2) is due to $S^{\lambda} V$ being irreducible yet $\left(S^{\lambda} V\right)^{\mathrm{SL}(V)}$ is an $\mathrm{SL}(V)$-invariant subspace, while the converse is from the fact that every character of $\operatorname{GL}(n)$ is of the form $\operatorname{det}_{V}^{r}$ for some $r \epsilon$ $\mathbb{Z}$ (since characters are, by definition, one-dimensional and we previously saw that all one-dimensional representations are powers of the determinant). The following propositions are immediate consequences of this result, hom-tensor adjointness, and Schur's Lemma.

Proposition 2.13. Let $\lambda, \mu \in \mathcal{P}_{n}$. Then

$$
\left(S^{\lambda} V^{*} \otimes S^{\mu} V\right)^{\mathrm{SL}(V)} \neq 0 \Leftrightarrow \operatorname{Hom}_{\mathrm{SL}(V)}\left(S^{\lambda} V, S^{\mu} V\right) \neq 0 .
$$

In this case, $S^{\lambda} V \cong S^{\mu} V$ as $\mathrm{SL}(V)$-modules, and $\left(S^{\lambda} V^{*} \otimes S^{\mu} V\right)^{\mathrm{SL}(V)}$ is a onedimensional vector space spanned by a semi-invariant of weight $k$.

Proposition 2.14. Let $\lambda, \mu \in \mathcal{P}_{n}$. Then

$$
\left(S^{\lambda} V \otimes S^{\mu} V\right)^{\mathrm{SL}(V)} \neq 0 \Leftrightarrow \operatorname{Hom}_{\mathrm{SL}(V)}\left(S^{\lambda} V^{*}, S^{\mu} V\right) \neq 0 \Leftrightarrow S^{\lambda} V^{*} \cong S^{\mu} V
$$

where the isomorphism is as $\mathrm{SL}(V)$-modules. In this case, $\lambda_{m+1-i}+\mu_{i}=k \in \mathbb{Z}$ for all $i$ (so $\lambda$ and $\mu$ fit together to form an $n \times k$ rectangle when one is rotated $180^{\circ}$ degrees), and $\left(S^{\lambda} V \otimes S^{\mu} V\right)^{\mathrm{SL}(V)}$ is one-dimensional and spanned by a semi-invariant of weight $k$. 
The next formula [Ful97a, p. 121] will allow us to compute semi-invariants for quivers, in conjunction with the preceding propositions. Recall that if $V$ is a finitedimensional vector space, then $S(V)$ denotes the symmetric algebra of $V$, that is, the algebra $\mathbb{C}\left[V^{*}\right]$ of polynomial functions on the dual space $V^{*}$. The Cauchy-Littlewood formula on symmetric functions implies

$$
\operatorname{Sym}^{k}(V \otimes W) \cong \bigoplus_{\lambda \vdash k} S^{\lambda}(V) \otimes S^{\lambda}(W)
$$

for two finite-dimensional vector spaces $V$ and $W$, where the sum ranges over all partitions $\lambda$ of $k$. Thus, we have the following formula because $S(V)=\oplus_{k} \operatorname{Sym}^{k}(V)$.

Proposition 2.15 (Cauchy's formula). Let $V$ and $W$ be finite-dimensional vector spaces. Then

$$
S(V \otimes W) \cong \bigoplus_{\lambda} S^{\lambda}(V) \otimes S^{\lambda}(W)
$$

as $\mathrm{GL}(V) \times \mathrm{GL}(W)$-representations, where the sum is over all partitions $\lambda$.

By Example 2.6(c), $S^{\lambda} V=S^{\lambda} W=0$ when the number of nonzero parts of $\lambda$ is greater than $\max \{\operatorname{dim} V, \operatorname{dim} W\}$.

Definition 2.16. Given three weakly decreasing sequences $\lambda(1), \lambda(2), \lambda(3)$ of $n$ integers, define the Littlewood-Richardson coefficient $c_{\lambda(1), \lambda(3)}^{\lambda(2)}$ to be the multiplicity of $S^{\lambda(2)}\left(\mathbb{C}^{n}\right)$ in $S^{\lambda(1)}\left(\mathbb{C}^{n}\right) \otimes S^{\lambda(3)}\left(\mathbb{C}^{n}\right)$. Equivalently,

$$
\begin{aligned}
c_{\lambda(1), \lambda(3)}^{\lambda(2)} & =\operatorname{dim}_{\mathbb{C}} \operatorname{Hom}_{\mathrm{GL}\left(\mathbb{C}^{n}\right)}\left(S^{\lambda(2)}\left(\mathbb{C}^{n}\right), S^{\lambda(1)}\left(\mathbb{C}^{n}\right) \otimes C^{\lambda(3)}\left(\mathbb{C}^{n}\right)\right) \\
& =\operatorname{dim}_{\mathbb{C}}\left(S^{\lambda(2)}\left(\mathbb{C}^{n}\right)^{*} \otimes S^{\lambda(1)}\left(\mathbb{C}^{n}\right) \otimes S^{\lambda(3)}\left(\mathbb{C}^{n}\right)\right)^{\mathrm{GL}\left(\mathbb{C}^{n}\right)} .
\end{aligned}
$$

By a dimension argument, $c_{\lambda(1), \lambda(3)}^{\lambda(2)}=0$ unless $|\lambda(2)|=|\lambda(1)|+|\lambda(3)|$. While it is extremely difficult to calculate these multiplicities in general (see Subsection 2.1.2 for a discussion of this), the Littlewood-Richardson rule provides a method in the case 
that the sequences are in fact partitions. For $\lambda, \mu, \nu \in \mathcal{P}_{n}$, a skew tableau of shape $\nu / \lambda$ is a Young diagram of shape $\nu$ with the first $\lambda_{i}$ consecutive boxes missing in row $i$. It has content $\mu$ if the associated tableau has $\mu_{i} i$ 's. The tableau satisfies the reverse lattice word condition if the content of the tableau, read from top to bottom, right to left, has at least as many $i$ 's as $(i+1)$ 's at any time.

Proposition 2.17. (Littlewood-Richardson rule) When $\lambda(i) \in \mathcal{P}_{n}, i \in\{1,2,3\}$, $c_{\lambda(1), \lambda(3)}^{\lambda(2)}$ is the number of semistandard Young tableaux of skew shape $\lambda(2) / \lambda(1)$ and content $\lambda(3)$ which satisfy the reverse lattice word condition. Equivalently, it is the number of semistandard Young tableaux of skew shape $\lambda(2) / \lambda(3)$ and content $\lambda(1)$ which satisfy the reverse lattice word condition.

A more in-depth discussion of this rule may be found in Chapter 5 of [Ful97a] and its proof is found in [Mac15].

Example 2.18. Suppose $\lambda(1)=(3,2), \lambda(2)=(4,3,2,1), \lambda(3)=(2,2,1)$. Consider
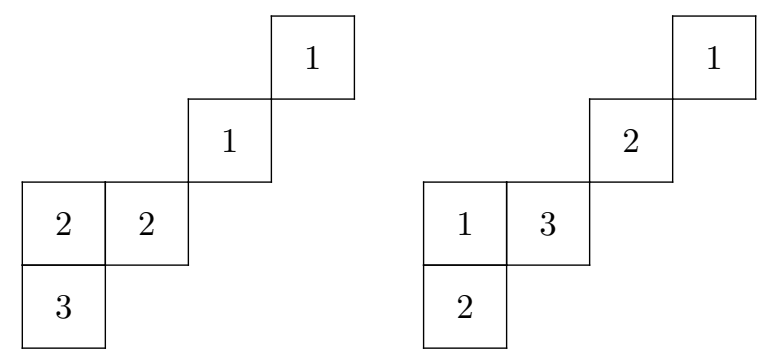

These are semistandard skew tableaux of shape $\lambda(2) / \lambda(1)$ and content $\lambda(3)$. The associated reverse lattice words are $(1,1,2,2,3)$ and $(1,2,3,1,2)$, both of which satisfy the condition. It's easily seen that these are the only two such semistandard skew tableaux of shape $\lambda(2) / \lambda(1)$ and content $\lambda(3)$ which satisfy the reverse lattice word 
condition. Thus, $c_{(3,2),(2,2,1)}^{(4,3,2,1)}=2$. Note that
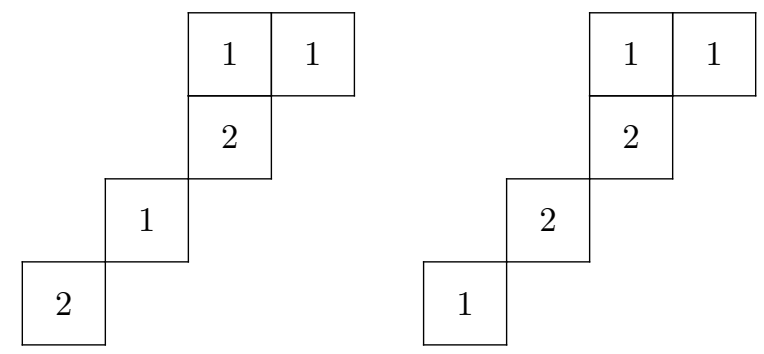

are semistandard Young tableaux of shape $\lambda(2) / \lambda(3)$ and content $\lambda(1)$ which again satisfy the reverse lattice word condition, and are the only such, so $c_{(2,2,1),(3,2)}^{(4,3,2,1)}=2$ as well.

We end this section with some properties of Littlewood-Richardson coefficients, which may be found in [Mac15].

Proposition 2.19. Let $\lambda, \mu, \nu$ be weakly decreasing sequences of $n$ integers. Then

$$
c_{\lambda, \mu}^{\nu}=c_{\mu, \lambda}^{\nu}=c_{\lambda^{\prime}, \mu^{\prime}}^{\nu^{\prime}}=c_{\widetilde{\nu}, \widetilde{\mu}}^{\widetilde{\lambda}}
$$

where $\lambda^{\prime}$ denotes the conjugate partition of $\lambda$ and $\widetilde{\lambda}=\left(m-\lambda_{n}, \ldots, m-\lambda_{1}\right)$ for any $m \in \mathbb{Z}$.

If a representation $W$ of $\operatorname{GL}(V)$ has highest weight $\lambda=\left(\lambda_{1}, \ldots, \lambda_{n}\right)$, then the highest weight of $W^{*}$ is $\left(-\lambda_{n}, \ldots,-\lambda_{1}\right)$, so $\widetilde{\lambda}$ is the highest weight of the representation $W^{*} \otimes \operatorname{det}_{V}^{m}$

We will need the following two lemmas in Subsection 4.2.2 when we discuss stretched functions. Both follow quickly from the Littlewood-Richardson rule (2.17).

Lemma 2.20. Let $\lambda, \mu$ be partitions and $\nu=\left(N^{n}\right)$ a rectangular partition. Then $c_{\lambda, \mu}^{\nu}$ is either 0 or 1 . It is equal to 1 if and only if $\lambda_{i}+\mu_{n+1-i}=N$ for $i=1, \ldots, n$. 
Proof. The Littlewood-Richardson rule states that $c_{\lambda, \mu}^{\nu}$ is the number of semistandard skew-tableau of shape $\nu / \lambda$ and content $\mu$ such that the associated word is a reverse lattice permutation. Clearly, $|\lambda|+|\mu|=|\nu|$ is a necessary condition for $c_{\lambda, \mu}^{\nu}$ to be nonzero, which for the case of $\nu=\left(N^{n}\right)$ means $\lambda_{i}+\mu_{n+1-i}=N$ for all $i$. Conversely, if this complementary condition is satisfied, then the first row in the skew tableau must consist of all 1's. To be semistandard, each entry below the 1's in the first row must be all 2's, after which the remaining entries in the second row (from right to left) must be 1's because of the reverse lattice word condition. Continuing in this way, the first $i$ occurs in row $i$, in which each $i$ occurs directly below each $i-1$ in the previous row, after which (reading from right to left) the next entries in row $i$ are $i-1$ and each occurs directly below the $i-2$ 's in the previous row. In all, the rightmost column of $\nu / \lambda$, from top to bottom, has entries $1,2, \ldots, \mu_{1}^{\prime}$, the next column to the left has entries $1,2, \ldots, \mu_{2}^{\prime}$, and so on. Because all of the entries are uniquely determined and this is a reverse lattice word, we have $c_{\lambda, \mu}^{\nu}=1$.

Lemma 2.21. Consider the rectangular partitions $\lambda=\left(a^{s}\right), \mu=\left(b^{t}\right)$. Assume $s \geqslant t$. Then for all partitions $\nu, c_{\lambda, \mu}^{\nu}$ is either 0 or 1 . Moreover, the value is 1 if and only if $\nu=\left(\nu_{1}, \ldots, \nu_{s+t}\right)$, where $\nu_{i}=a+c_{i}$ for $1 \leqslant i \leqslant t$ and $b \geqslant c_{1} \geqslant \ldots \geqslant c_{t} \geqslant 0, \nu_{i}=a$ for $t<i \leqslant s$, and $\nu_{s+i}=b-c_{t+1-i}$ for $1 \leqslant i \leqslant t$.

Proof. The proof of this is similar to that of Lemma 2.20. It is immediate that because we have rectangular partitions, the only semistandard tableaux with weight a reverse lattice word will have all 1's in the first row, then 2's in the second row (read right to left) until we're to the left of the 1's in the first row, at which point the remaining entries will become 1's. The third row will start (from the right) with 
3's and continue the weakly decreasing pattern as determined the second row. The entries in the rightmost column of the filled skew tableau $\nu / \lambda$ will then be (read top to bottom) $1,2, \ldots$, and similarly for each of the $\mu_{1}$ columns to the left, after which the entries will be $2,3, \ldots$, and so on. This is exactly the content of the statement.

\subsubsection{The complexity of Littlewood-Richardson coefficients}

Geometric complexity theory (GCT) was introduced by Mulmuley and Sohoni in a series of papers (see [MS07], [MS01a], [MS01b], [MS08], [MNS12], [MS17], [Mul10], [Mul11]) in the early 2000's with the purpose of approaching fundamental problems in complexity theory, such as $\mathrm{P}$ vs. NP, through algebraic geometry and representation theory. Previously, [KT01] and [LM06] had independently shown that the positivity of Littlewood-Richardson coefficients could be computed in polynomial time while [Nar05] had shown that the exact computation of these numbers was a \#P-complete problem, the complexity class for problems for which (unless $\mathrm{P}=\mathrm{NP}$ ) there does not exist a polynomial time algorithm for computing them (rather, it takes an exponential time in the worst case), and such that the computation is at least as difficult as every P problem.

The following is the main theorem of [MS05], where the bit length of a partition $\lambda=\left(\lambda_{1}, \ldots, \lambda_{k}\right), \lambda_{k}>0$, is the bit length of the specifications: $\sum_{i=1}^{k} \log _{2} \lambda_{i}$.

Theorem 2.22. Deciding whether $c_{\lambda, \mu}^{\nu}$ is positive can be computed in strongly polynomial time in the sense of [GLS93]. This means that the number of arithmetic steps is polynomial in the number of positive parts of $\nu$ (say $n)$, does not depend on the bit lengths of $\lambda_{i}, \mu_{j}, \nu_{k}$, and the bit length of every intermediate operand that arises in the algorithm is polynomial in the total bit length of $\lambda, \mu, \nu$. 
In fact, by attaching zeros to the partitions, one can subsume the dependence on $n$ into the bit lengths of $\lambda, \mu$, and $\nu$. This is especially amazing as the the dimension of the Schur module $S^{\nu} V$ is exponential in $n$ and the bit lengths of the $\nu_{k}$ 's, yet deciding if an exponential dimensional object $S^{\nu} V$ arises in the decomposition of another exponential dimensional object $S^{\lambda} V \otimes S^{\mu} V$ can be decided in time that is polynomial in only $n$ and the bit lengths of the labels $\lambda, \mu$, and $\nu$.

In order to determine the complexity of the positivity of the generalized LittlewoodRichardson coefficients, we will use the combinatorial gadgets developed by Knutson and Tao [KT99] in their proof of the saturation conjecture (Theorem 1.1), which they along with C. Woodward also used to provide combinatorial proofs of the complexity of the positivity along with various properties of Littlewood-Richardson coefficients, such as some listed in Proposition 2.19 [KTW04]. While their proof used what they called "honeycombs," we'll have no need for these. Indeed, Buch [Buc00] showed that their hive models suffice.

We recall their construction of LR hives in this section. Their process rests on a common approach in combinatorics: to understand the properties of some multiplicity, define a polytope in a way that the number of lattice points in the polytope is precisely that multiplicity and then use geometric techniques to study the polytope. To define the polytope whose number of lattice points is the Littlewood-Richardson coefficient $c_{\lambda, \mu}^{\nu}$ for a specific choice of partitions $\nu, \lambda$, and $\mu$ with at most $n$ parts, subdivide a triangle into $n^{2}$ smaller triangles of the same size by plotting $n+1$ vertices along each edge of the large triangle. Label the edges in the following way: the first subscript $i$ refers to the row from bottom to top while the second subscript $j$ refers 
to the diagonal from left to right, and $0 \leqslant i, j \leqslant n-1$. The edges along increasing diagonals are labeled $e_{i j}$, the edges along decreasing diagonals are labeled $f_{i j}$, and the horizontal edges are $g_{i j}$. We call such a labeling a hive model. For instance, when $n=3$ the triangular array is labeled

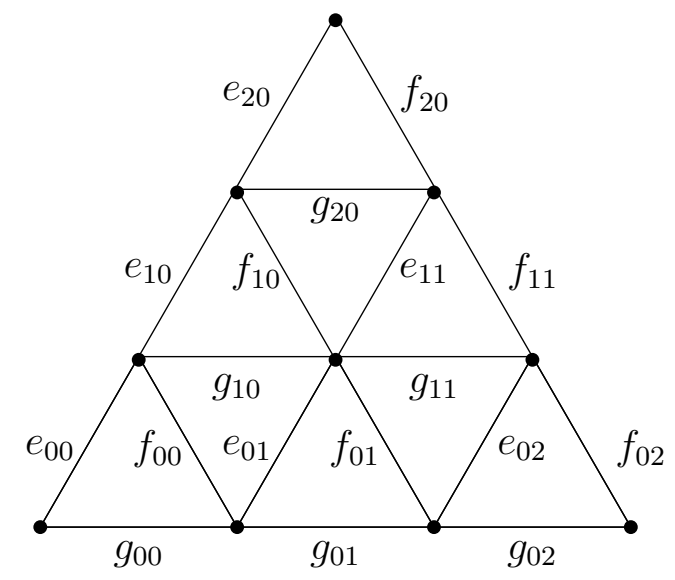

Let $E$ be the set of hive edges and $\mathbb{R}^{E}$ the labelings of these edges by real numbers. There are three ways that two adjacent triangles inside a triangular array can form a rhombus:
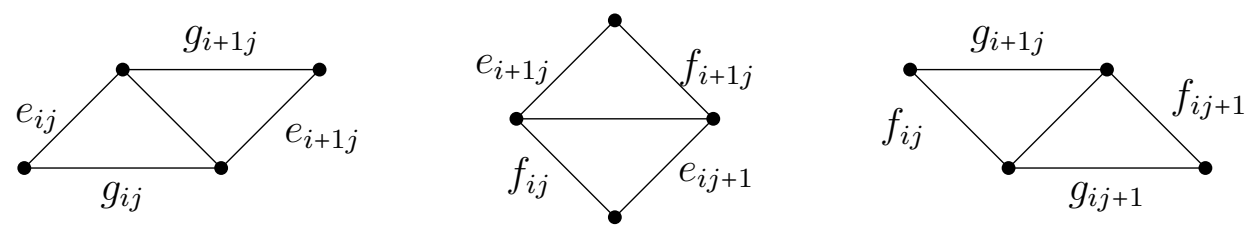

We say these rhombi satisfy the rhombus inequalities if for each triangle and rhombus appearing, we have

$$
\begin{gathered}
e_{i j} \geqslant e_{i j+1}, \quad g_{i j} \geqslant g_{i+1 j} ; \quad f_{i+1 j} \geqslant f_{i j}, \quad e_{i j+1} \geqslant e_{i+1 j} ; \quad f_{i j} \geqslant f_{i j+1}, \quad g_{i+1 j} \geqslant g_{i j+1} \\
e_{i j}+f_{i j}=g_{i j}, \quad e_{i j+1}+f_{i j}=g_{i+1 j} .
\end{gathered}
$$


It is integral if the labeling lies in $\mathbb{Z}^{E}$. These inequalities define a convex polyhedral cone, denoted $C \subseteq \mathbb{R}^{E}$. An $\boldsymbol{L} \boldsymbol{R}$ hive is a triangular array that satisfies the rhombus inequalities and border conditions

$$
\sum_{i=0}^{n-1} e_{i 0}+\sum_{i=0}^{n-1} f_{i n-i}=\sum_{j=0}^{n-1} g_{0 j}
$$

Let $B$ be the set of border edges $g_{0 j}$ for $0 \leqslant j \leqslant n-1$, and $\rho: \mathbb{R}^{E} \rightarrow \mathbb{R}^{B}$ the restriction map of an LR hive to its border. For each $b \in \mathbb{R}^{B}$, the fiber $\rho^{-1}(b) \cap C$ is a compact polytope, called the hive polytope over $b$.

We recall the main result of [KT99]. For three $n$-tuples

$$
\lambda=\left(\lambda_{1}, \ldots, \lambda_{n}\right), \quad \mu=\left(\mu_{1}, \ldots, \mu_{n}\right), \quad \nu=\left(\nu_{1}, \ldots, \nu_{n}\right)
$$

that satisfy the boundary condition $|\nu|=|\lambda|+|\mu|$, the triangular array with border determined by $\lambda, \mu, \nu$ is the one with specified border edges

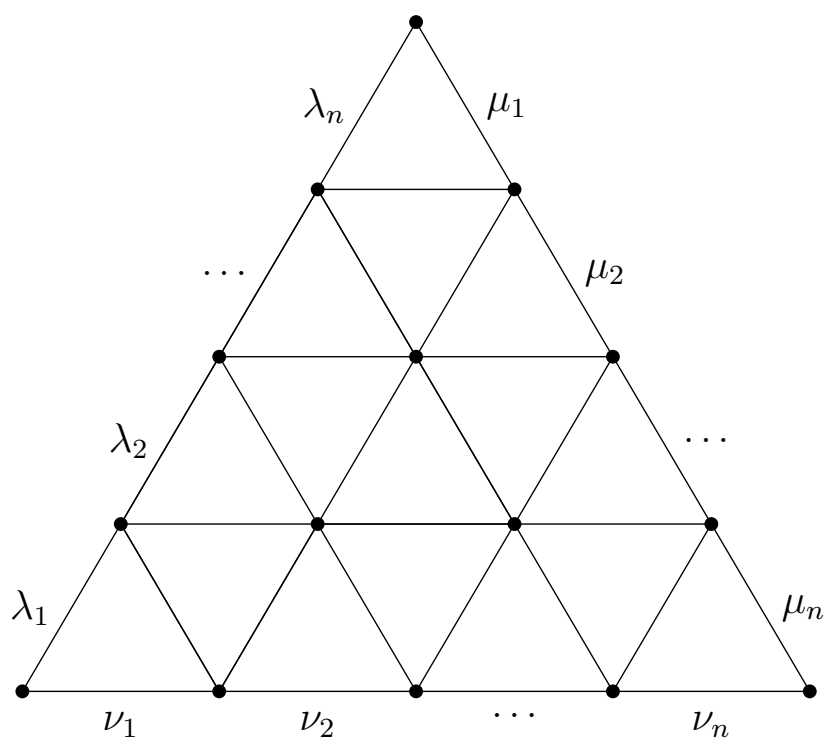

Theorem 2.23 ([KT99], Theorem 4). The Littlewood-Richardson coefficient $c_{\lambda, \mu}^{\nu}$ is the number of integer LR hives with boundary labels determined by $\lambda, \mu$, and $\nu$. 
This theorem is what allowed Knutson and Tao to prove the saturation theorem, while the complexity of the positivity of Littlewood-Richardson coefficients follows from the saturation theorem and the polyhedral description.

Because we are only interested in integer LR hives it suffices to restrict to when $\lambda, \mu, \nu$ are weakly decreasing sequences of integers. Further, the saturation for polynomial representations is easily seen to be equivalent to the saturation for rational representations by tensoring with a high enough power of the determinant representation, so we may consider simply partitions rather than all weakly decreasing sequences of integers.

Though the border conditions are obvious from the necessary condition that $|\nu|=$ $|\lambda|+|\mu|$ for $c_{\lambda, \mu}^{\nu}$ to be nonzero, the rhombus inequalities seem mysterious at first. Their inspiration comes from Gelfand-Tsetlin patterns and Cauchy's Interlacing Theorem for the eigenvalues of Hermitian matrices. The first two pairs of inequalities ensure that the tuples are weakly decreasing while the third pair gives a way of associating a contratableau satisfying the Littlewood-Richardson rule; for more on this, see [Buc00].

We will lastly need a characterization of the vertices a polytope defined by homogeneous linear inequalities. Every closed convex set is the intersection of affine halfspaces, and the convex set is called a polyhedron when it is the intersection of finitely many affine halfspaces. Hence, a subset $P \subseteq \mathbb{R}^{n}$ is a polyhedron if and only if there is an $m \times n$ matrix $A$ and a vector $\mathbf{b} \in \mathbb{R}^{m}$ such that $P=\left\{\mathbf{x} \in \mathbb{R}^{n} \mid A \mathbf{x} \leqslant \mathbf{b}\right\}$. A subset $P \subseteq \mathbb{R}^{n}$ is called a polytope if $P$ is the convex hull of finitely many vectors. The relationship between the two is that $P$ is a polytope if and only if it is a bounded polyhedron. A point $\mathbf{v} \in P$ is called a vertex if it is not a convex combination of any 
two other points in $P$, that is, there are not points $\mathbf{x}, \mathbf{y} \in P$ and $0<\lambda<1$ such that $\mathbf{v}=\lambda \mathbf{x}+(1-\lambda) \mathbf{y}$. The following is a standard result in combinatorial optimization (see $[$ Sch03]).

Lemma 2.24. Let $P=\{\mathbf{x} \mid A \mathbf{x} \leqslant \mathbf{b}\}$ be a polyhedron in $\mathbb{R}^{n}$ and let $\mathbf{v} \in P$. Then $\mathbf{v}$ is a vertex of $P$ if and only if $\operatorname{rank}\left(A_{\mathbf{v}}\right)=n$, where $A_{\mathbf{v}}$ is the submatrix of $A$ consisting of rows $\mathbf{a}_{\mathbf{i}}$ of $A$ for which $\mathbf{a}_{\mathbf{i}} \mathbf{v}=b_{i}$. In particular, $\mathbf{v}$ is a vertex of $P$ if and only if $A \mathbf{v}=\mathbf{b}$.

Proof. First suppose $\operatorname{rank}\left(A_{\mathbf{v}}\right)<n$. Then there is a vector $\mathbf{c} \neq 0$ such that $A_{\mathbf{v}} \mathbf{c}=\mathbf{0}$. Since $\mathbf{a}_{\mathbf{i}} \mathbf{v}<b_{i}$ for every $\mathbf{a}_{\mathbf{i}}$ that isn't in $A_{\mathbf{v}}$, there is some $\delta>0$ such that

$$
\mathbf{a}_{\mathbf{i}}(\mathbf{v}+\delta \mathbf{c}) \leqslant b_{i} \quad \text { and } \quad \mathbf{a}_{\mathbf{i}}(\mathbf{v}-\delta \mathbf{c}) \leqslant b_{i}
$$

for every row $\mathbf{a}_{\mathbf{i}}$ of $A$ not in $A_{\mathbf{v}}$. Because $A_{\mathbf{v}} \mathbf{c}=\mathbf{0}$ and $A \mathbf{v} \leqslant \mathbf{b}$, we have

$$
A(\mathbf{v}+\delta \mathbf{c}) \leqslant \mathbf{b} \quad \text { and } \quad A(\mathbf{v}-\delta \mathbf{c}) \leqslant \mathbf{b} .
$$

Thus, $\mathbf{v}+\delta \mathbf{c}$ and $\mathbf{v}-\delta \mathbf{c}$ belong to $P$, so $\mathbf{v}$ is a linear combination of two points in $P$, and hence is not a vertex.

Conversely, if $\mathbf{v}$ is not a vertex, then there exist points $\mathbf{x}, \mathbf{y} \in P$ such that $\mathbf{x} \neq \mathbf{v} \neq \mathbf{y}$ and $\mathbf{v}=(\mathbf{x}+\mathbf{y}) / 2$. Then for every row $\mathbf{a}_{\mathbf{i}}$ in $A_{\mathbf{v}}$

$$
\mathbf{a}_{\mathbf{i}} \mathbf{x} \leqslant b_{i}=\mathbf{a}_{\mathbf{i}} \mathbf{V} \Rightarrow \mathbf{a}_{\mathbf{i}}(\mathbf{x}-\mathbf{v}) \leqslant 0 \quad \text { and } \quad \mathbf{a}_{\mathbf{i}} \mathbf{y} \leqslant b_{i}=\mathbf{a}_{\mathbf{i}} \mathbf{v} \Rightarrow \mathbf{a}_{\mathbf{i}}(\mathbf{y}-\mathbf{v}) \leqslant 0 .
$$

Because $\mathbf{y}-\mathbf{v}=-(\mathbf{x}-\mathbf{v})$, it follows that $\mathbf{a}_{\mathbf{i}}(\mathbf{x}-\mathbf{v})=0$. Thus, $A_{\mathbf{v}}(\mathbf{x}-\mathbf{v})=\mathbf{0}$. Because $\mathbf{x}-\mathbf{v} \neq \mathbf{0}$, this implies $\operatorname{rank}\left(A_{\mathbf{v}}\right)<n$. 


\subsection{Quiver theory}

\subsubsection{Preliminaries}

A quiver $Q=\left(Q_{0}, Q_{1}, t, h\right)$ consists of a finite set of vertices $Q_{0}$, a finite set of arrows $Q_{1}$, and functions $t, h: Q_{1} \rightarrow Q_{0}$ that assign the tail $t a$ and head $h a$ of each arrow $a$, commonly denoted $t a \stackrel{a}{\rightarrow} h a$. Note that we allow multiple arrows between two vertices and loops in the directed graph $Q$.

Throughout this thesis we always work over the complex numbers $\mathbb{C}$. A representation $V$ of $Q$ is a family of finite-dimensional vector spaces (over $\mathbb{C})\left\{V(x) \mid x \in Q_{0}\right\}$ together with a family of linear transformations $\left\{V(a): V(t a) \rightarrow V(h a) \mid a \in Q_{1}\right\}$. For a representation $V$, its dimension vector $\underline{\operatorname{dim}} V$ is defined by $\underline{\operatorname{dim}} V(x)=\operatorname{dim}_{\mathbb{C}} V(x)$ for all $x \in Q_{0}$. The dimension vectors of representations of $Q$ then lie in $\Gamma=\mathbb{Z}^{Q_{0}}$, the set of integer-valued functions on $Q_{0}$. For each vertex $x \in Q_{0}$, there is a simple

representation $S_{x}$ defined by the dimension vector $e_{x}(y)=\delta_{x, y}$ for all $y \in Q_{0}$, where $\delta_{x, y}$ is the Kronecker delta.

Given two representations $V$ and $W$ of $Q$, define a morphism $\phi: V \rightarrow W$ of representations to be a collection of linear maps $\left\{\phi(x): V(x) \rightarrow W(x) \mid x \in Q_{0}\right\}$ such that for every arrow $a \in Q_{1}$ we have $\phi(h a) V(a)=W(a) \phi(t a)$, meaning the diagram

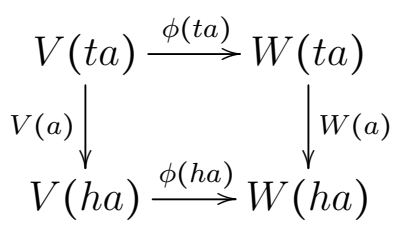

commutes. Define $\operatorname{Hom}_{Q}(V, W)$, or simply $\operatorname{Hom}(V, W)$, to be the $\mathbb{C}$-vector space of all morphisms from $V$ to $W$. We thus obtain the abelian category $\operatorname{Rep}(Q)$ of all quiver representations of $Q$. We denote the subcategory of all finite-dimensional representations by $\operatorname{rep}(Q)$. We call $V^{\prime}$ a subrepresentation of $V$ if $V^{\prime}(x)$ is a 
subspace of $V(x)$ for all vertices $x \in Q_{0}$ and $V^{\prime}(a)=\left.V(a)\right|_{V^{\prime}(t a)}$ for all arrows $a \in Q_{1}$.

For any $\alpha, \beta \in \Gamma$, define the Euler form by

$$
\langle\alpha, \beta\rangle=\sum_{x \in Q_{0}} \alpha(x) \beta(x)-\sum_{a \in Q_{1}} \alpha(t a) \beta(h a) .
$$

The Euler form has a particularly nice homological interpretation for quivers without cycles or relations. In this setting, the category $\operatorname{Rep}(Q)$ is hereditary, meaning that subobjects of projective objects are themselves projective. Equivalently, $\operatorname{Ext}_{Q}^{i}(V, W)=0$ for all $V, W \in \operatorname{Rep}(Q)$ and $i>1$. Ringel [Rin76] provided a canonical projective resolution of each $V \in \operatorname{Rep}(Q)$ by using that the map

$$
d_{W}^{V}: \bigoplus_{x \in Q_{0}} \operatorname{Hom}(V(x), W(x)) \rightarrow \bigoplus_{a \in Q_{1}} \operatorname{Hom}(V(t a), W(h a))
$$

defined by

$$
d_{W}^{V}\left((\phi(x))_{x \in Q_{0}}\right)=(\phi(h a) V(a)-W(a) \phi(t a))_{a \in Q_{1}}
$$

has kernel $\operatorname{Hom}_{Q}(V, W)$ and cokernel $\operatorname{Ext}_{Q}^{1}(V, W)$, respectively. Hence, for quivers without oriented cycles, the Euler form evaluated on dimension vectors becomes

$$
\langle\underline{\operatorname{dim}} V, \underline{\operatorname{dim}} W\rangle=\operatorname{dim}_{\mathbb{C}} \operatorname{Hom}_{Q}(V, W)-\operatorname{dim}_{\mathbb{C}} \operatorname{Ext}_{Q}^{1}(V, W) .
$$

\subsubsection{Semi-invariants for quivers}

For a dimension vector $\beta$ of a quiver $Q$, the representation space of $\beta$-dimensional representations of $Q$ is defined as

$$
\operatorname{Rep}(\mathrm{Q}, \beta)=\bigoplus_{a \in Q_{1}} \operatorname{Hom}\left(\mathbb{C}^{\beta(t a)}, \mathbb{C}^{\beta(h a)}\right)
$$

Note that this is simply an affine space. If $\operatorname{GL}(\beta)=\prod_{x \in Q_{0}} \operatorname{GL}(\beta(x))$, then there is a natural action of $\operatorname{GL}(\beta)$ on $\operatorname{Rep}(\mathrm{Q}, \beta)$ given by simultaneous conjugation: for 
$g=(g(x))_{x \in Q_{0}} \in \mathrm{GL}(\beta)$ and $V=\{V(a)\}_{a \in Q_{1}} \in \operatorname{Rep}(\mathrm{Q}, \beta), g \cdot V$ is defined by

$$
(g \cdot V)(a)=g(h a) V(a) g(t a)^{-1} \quad \forall a \in Q_{1}
$$

Hence, $\operatorname{Rep}(\mathrm{Q}, \beta)$ is a rational representation of the linearly reductive group $\operatorname{GL}(\beta)$ and the $\mathrm{GL}(\beta)$-orbits parameterize the isomorphism classes of $\beta$-dimension representations of $Q$ since the action is simply base change (with respect to a specified basis). If $Q$ is without oriented cycles, there is only one closed $\operatorname{GL}(\beta)$-orbit in $\operatorname{Rep}(\mathrm{Q}, \beta)$ (specif-

ically, the orbit of the unique $\beta$-dimensional semisimple representation $\left.\bigoplus_{x \in Q_{0}} S_{x}^{\beta(x)}\right)$, so the invariant ring $\mathbb{C}[\operatorname{Rep}(\mathrm{Q}, \beta)]^{\mathrm{GL}(\beta)}$ is simply $\mathbb{C}$. However, while there are only constant $\operatorname{GL}(\beta)$-invariant polynomial functions on $\operatorname{Rep}(\mathrm{Q}, \beta)$, the action descends to that of the subgroup $\operatorname{SL}(\beta)$, and the invariant ring under the action of this group is highly nontrivial.

Let $\operatorname{SI}(Q, \beta)=\mathbb{C}[\operatorname{Rep}(\mathrm{Q}, \beta)]^{\mathrm{SL}(\beta)}$ be the ring of semi-invariants. Since $\mathrm{GL}(\beta)$ is linearly reductive and $\operatorname{SL}(\beta)$ is the commutator subgroup of $\operatorname{GL}(\beta)$, we have the weight space decomposition

$$
\operatorname{SI}(Q, \beta)=\bigoplus_{\sigma \in X^{*}(\operatorname{GL}(\beta))} \operatorname{SI}(Q, \beta)_{\sigma}
$$

where $X^{*}(\mathrm{GL}(\beta))$ is the group of rational characters of $\operatorname{GL}(\beta)$ and

$$
\operatorname{SI}(Q, \beta)_{\sigma}=\{f \in \mathbb{C}[\operatorname{Rep}(\mathrm{Q}, \beta)] \mid g \cdot f=\sigma(g) f \forall g \in \operatorname{GL}(\beta)\}
$$

is the space of semi-invariants of weight $\sigma$. A character (or weight) of GL $(\beta)$ is of the form

$$
\left\{g(x) \mid x \in Q_{0}\right\} \in \mathrm{GL}(\beta) \mapsto \prod_{x \in Q_{0}}(\operatorname{det} g(x))^{\sigma(x)}
$$

for $\sigma(x) \in \mathbb{Z}$ for all $x \in Q_{0}$, so we may identify $X^{*}(\operatorname{GL}(\beta))$ with $\mathbb{Z}^{Q_{0}}$ because all 
one-dimensional representations of $\mathrm{GL}(n)$ are some power of the determinant representation for any $n$. For an integer-valued function $\alpha$ on $Q_{0}$, define $\sigma=\langle\alpha, \cdot\rangle$ by

$$
\sigma(x)=\left\langle\alpha, e_{x}\right\rangle=\alpha(x)-\sum_{y \rightarrow x} \alpha(y), \quad \forall x \in Q_{0} .
$$

One can similarly define $\sigma=\langle\cdot, \alpha\rangle$.

Given a quiver $Q$ and dimension vector $\beta$, define the set $\Sigma(Q, \beta)$ to be the set of integral effective weights:

$$
\Sigma(Q, \beta)=\left\{\sigma \in \mathbb{Z}^{Q_{0}} \mid \operatorname{SI}(Q, \beta)_{\sigma} \neq 0\right\} .
$$

Schofield [Sch91] constructed distinguished semi-invariants for quivers that proved to be quite useful in studying the ring of semi-invariants. Specifically, if $\alpha, \beta \in \mathbb{N} Q_{0}$ are dimension vectors such that $\langle\alpha, \beta\rangle=0$, then the matrix of $d_{W}^{V}$ in Equation 2.4 is a square matrix for any $V \in \operatorname{rep}(Q, \alpha), W \in \operatorname{rep}(Q, \beta)$. Then $c(V, W):=\operatorname{det} d_{W}^{V}$ is a semi-invariant of the action of $\operatorname{GL}(\alpha) \times \operatorname{GL}(\beta)$ on $\operatorname{rep}(Q, \alpha) \times \operatorname{rep}(Q, \beta)$. For a fixed $V$, Schofield [Sch91] proved that the restriction to $\{V\} \times \operatorname{rep}(Q, \beta)$ defines a semi-invariant $c^{V}$ of weight $\langle\alpha, \cdot\rangle$ in $\operatorname{SI}(Q, \beta)$. Similarly, for a fixed $W$, we get a semiinvariant $c_{W}$ of weight $-\langle\cdot, \beta\rangle$ in $\operatorname{SI}(Q, \alpha)$. Derksen and Weyman (see also [SB01]) showed that these semi-invariants in fact span all spaces of semi-invariants.

Theorem 2.25 ([DW00a], Theorem 1$)$. The ring $\operatorname{SI}(Q, \beta)$ is spanned by the semiinvariants $c^{V}$ for which $\langle\underline{\operatorname{dim}} V, \beta\rangle=0$. It is also spanned by the semi-invariants $c_{W}$ for which $\langle V, \underline{\operatorname{dim}} W\rangle=0$.

An important consequence of this result is the following saturation property. 
Theorem 2.26 ([DW00a], Theorem 3). If $Q$ is a quiver without oriented cycles and $\beta$ is a dimension vector, then the set

$$
\Sigma(Q, \beta)=\left\{\sigma \in \mathbb{Z}^{Q_{0}} \mid \operatorname{SI}(Q, \beta)_{\sigma} \neq 0\right\}
$$

is saturated, that is, if $\sigma$ is a weight and $r \geqslant 1$ an integer,

$$
\operatorname{SI}(Q, \beta)_{\sigma} \neq 0 \Longleftrightarrow \operatorname{SI}(Q, \beta)_{r \sigma} \neq 0 .
$$

We will later use this theorem to prove the saturation of the multiplicities (1.2) and (1.3) and give an explicit description of the nonzero generalized LittlewoodRichardson coefficients of these forms; see Propositions 3.19 and 3.24 and Lemma 3.25 .

Example 2.27. We provide some examples of the computation of semi-invariants. These examples anticipate the calculation of the rings of semi-invariants in Chapter 3 in which we provide more details of the calculations.

1. Consider the quiver $Q=\overrightarrow{A_{1}}=1 \stackrel{a}{\rightarrow} 2$ and dimension vector $\beta=(m, n)$. We want to describe the ring of semi-invariants $\operatorname{SI}(Q, \beta)$. Let $V=k^{m}, W=k^{n}$. Then $\operatorname{rep}(Q, \beta)=\operatorname{Hom}_{\mathbb{C}}(V, W) \cong V^{*} \otimes W$, so

$$
\mathbb{C}[\operatorname{rep}(Q, \beta)]=\mathbb{C}\left[V^{*} \otimes W\right]=S\left(V \otimes W^{*}\right)=\bigoplus_{\lambda \in \mathcal{P}} S^{\lambda} V \otimes S^{\lambda} V^{*}
$$

Hence, $\operatorname{SI}(Q, \beta)=\oplus_{\lambda \in \mathcal{P}}\left(\left(S^{\lambda} V\right)^{\mathrm{SL}(V)} \otimes\left(S^{\lambda} W^{*}\right)^{\mathrm{SL}(W)}\right)$ by Cauchy's formula (Proposition 2.15).

By Proposition 2.12, $\left(S^{\lambda} V\right)^{\mathrm{SL}(V)} \neq 0$ if and only if $\lambda=\left(k^{m}\right)$ for some $k \in \mathbb{Z}_{\geqslant 0}$. Similarly, $\left(S^{\lambda} W^{*}\right)^{\operatorname{SL}(W)} \neq 0$ if and only if $\lambda=\left(l^{n}\right)$ for some $l \in \mathbb{Z}_{\geqslant 0}$. If $m \neq n$, then clearly all such tensor products have at least one zero factor other than the 
trivial representation, so the only semi-invariants are the constants. If $m=n$, then $k=l$ and because each semi-invariant factor is one dimensional of weight $k$ or $-k$, then $\operatorname{SI}(Q, \beta)=\bigoplus_{k \geqslant 0} \operatorname{det}_{V}^{k} \otimes \operatorname{det}_{W}^{-k}$. Thus, $\operatorname{SI}(Q, \beta)=k\left[\operatorname{det}_{a}\right]$ if $m=n$ and $\operatorname{SI}(Q, \beta)=k$ if $m \neq n$.

Similarly, if

$$
Q=\vec{A}_{n+1}: 1 \stackrel{a_{1}}{\longrightarrow} 2 \stackrel{a_{2}}{\longrightarrow} \cdots \stackrel{a_{n}}{\longrightarrow} n+1
$$

the ring of semi-invariants for the dimension vector $\beta=(m, m, \ldots, m)$ is $\operatorname{SI}(Q, \beta)=$ $k\left[\operatorname{det}_{a_{1}}, \ldots, \operatorname{det}_{a_{n}}\right]$.

2. Consider the Kronecker quiver $Q=1 \Longrightarrow 2$ and dimension vector $\beta=(n, m)$. A similar calculation yields

$$
\operatorname{SI}(Q, \beta)=\bigoplus_{\lambda, \mu \in \mathcal{P}}\left(\left(S^{\lambda} V \otimes S^{\mu} V\right)^{\mathrm{SL}(V)} \otimes\left(S^{\lambda} W^{*} \otimes S^{\mu} W^{*}\right)^{\mathrm{SL}(W)}\right) .
$$

From Proposition 2.14, we conclude that a factor is nonzero if and only if $\lambda$ and $\mu$ fit together to form an $n \times k$ rectangle as well as an $m \times l$ rectangle for some $k, l \in \mathbb{Z}_{\geqslant 0}$. If so, then it's spanned by a semi-invariant of weight $(k,-l)$.

In the case that $n \neq m$. Then $\lambda$ and $\mu$ can't fit together to form both rectangles, so the tensor product must be zero. Hence, $\operatorname{SI}(Q, \beta)=\mathbb{C}$ in this case. If, however, $n=m$, then $k=l$ and $\operatorname{dim} \operatorname{SI}(Q, \beta)_{(k,-k)}$ is equal to the number of partitions fitting into an $m \times k$ rectangle, which is $\left(\begin{array}{c}m+k \\ k\end{array}\right)$. Consider the function $\operatorname{rep}(Q, \beta) \rightarrow \mathbb{C}$ defined by

$$
(A, B) \mapsto \operatorname{det}(A x+B y)=\sum_{i=0}^{m} f_{i}(A, B) x^{i} y^{m-i} \in \mathbb{C}[\operatorname{rep}(Q, \beta)][x, y]
$$

It's easy to check that $f_{i} \in \operatorname{SI}(Q, \beta)_{(1,-1)}$ for all $i$. D. Happel [Hap84] proved that $f_{0}, \ldots, f_{m}$ are algebraically independent, allowing us to conclude (since 
the dimension cannot exceed $m+1)$ that $\operatorname{SI}(Q, \beta)=\mathbb{C}\left[f_{0}, \ldots, f_{m}\right]$ and that $\operatorname{SI}(Q, \beta)_{(k,-k)}=\mathbb{C}\left[f_{0}, \ldots, f_{m}\right]_{k}$ for any $k \in \mathbb{Z}_{\geqslant 0}$

3. Because of the complexity of the representation theory of the quiver $Q=1 \rightrightarrows 2$, no closed formulas are known for the associated rings of semi-invariants, not even in the case of $\alpha=(n, n)$ and weight $(k,-k)$.

\subsection{3 $\sigma$-semi-stability}

If $\sigma \in \mathbb{R}^{Q_{0}}$ is a real-valued function on the set of vertices $Q_{0}$ and $\alpha$ is an integer-valued function on $Q_{0}$, define $\sigma(\alpha)$ by

$$
\sigma(\alpha)=\sum_{x \in Q_{0}} \sigma(x) \alpha(x)
$$

The condition $\sigma(\beta)=0$ is clearly necessary for $\sigma$ to be effective. This is because the action of the one-dimensional torus $\left\{\left(t \operatorname{Id}_{\beta(i)}\right)_{i \in Q_{0}} \mid t \in k \backslash\{0\}\right\}$ on $\operatorname{Rep}(\mathrm{Q}, \beta)$ is trivial, so if $f$ is a nonzero semi-invariant of weight $\sigma$ and $g_{t}=\left(t \operatorname{Id}_{\beta(i)}\right)_{i \in Q_{0}} \in \operatorname{GL}(\beta)$, then

$$
g_{t} \cdot f=t^{\sigma(\beta)} \cdot f
$$

which implies $\sigma(\beta)=0$. Surprisingly, satisfying a certain set of linear homogenous inequalities is sufficient for a weight to be effective (see Theorems 2.32 and 2.42).

Definition 2.28. Let $W \in \operatorname{Rep}(\mathrm{Q}, \beta)$. We say that

1. $W$ is $\sigma$-semi-stable if there exists a semi-invariant $f \in \operatorname{SI}(Q, \beta)_{m \sigma}$ for some integer $m \geqslant 1$ such that $f(W) \neq 0$.

2. $W$ is $\sigma$-stable if there exists a semi-invariant $f \in \operatorname{SI}(Q, \beta)_{m \sigma}$ for some $m \geqslant 1$ such that $f(W) \neq 0, \operatorname{dim} \mathrm{GL}(\beta) W=\mathrm{GL}(\beta)-1$, and the action of $\mathrm{GL}(\beta)$ on the affine variety $\{X \in \operatorname{Rep}(\mathrm{Q}, \beta) \mid f(X) \neq 0\}$ is separated. 
While there are no interesting quotient varieties for the action of $\operatorname{GL}(\beta)$ on $\operatorname{Rep}(\mathrm{Q}, \beta)$, twisting the action by a weight $\sigma$ provides many nontrivial semi-invariants. By doing this, King [Kin94] gave the following numerical criterion for $\sigma$-(semi-)stability for finite-dimensional algebras based on the Hilbert-Mumford criterion from geometric invariant theory (GIT). (King's criterion differs in sign from our convention, which is why the inequalities in the following theorem go the opposite direction as the ones in his original paper.)

Theorem 2.29. Let $Q$ be a quiver, $\beta$ a dimension vector, and $V \in \operatorname{Rep}(\mathrm{Q}, \beta)$. Suppose $\sigma \in \mathbb{Z}^{Q_{0}}$ is a weight such that $\sigma(V)=0$. Then

1. $V$ is $\sigma$-semi-stable if and only if $\sigma\left(\underline{\operatorname{dim}} V^{\prime}\right) \leqslant 0$ for every subrepresentation $V^{\prime}$ of $V$;

2. $V$ is $\sigma$-stable if and only if $\sigma\left(\underline{\operatorname{dim}} V^{\prime}\right)<0$ for every proper nontrivial subrepresentation $V^{\prime}$ of $V$.

We call $\beta \sigma$-(semi)-stable if there exists a $\sigma$-(semi-) stable representation in $\operatorname{Rep}(\mathrm{Q}, \beta)$.

Remark 2.30. Because of this description of the $\sigma$-(semi)-stable representations, there is a full subcategory of $\operatorname{Rep}(Q)$ consisting $\sigma$-(semi)-stable representations. This is an abelian category with simple objects being the $\sigma$-stable representations. Moreover because every representation has finite length, the subcategory is Artinian and Noetherian, so any $\sigma$-semi-stable representation has a Jordan-Hölder filtration with $\sigma$-stable factors.

The following result is quite useful for calculations and is immediate from Theorem 2.25 .

Lemma 2.31 (Reciprocity Property). ([DW00a], Corollary 1) For any dimension 
vectors $\alpha, \beta$ and quiver $Q$ without oriented cycles, if $\langle\alpha, \beta\rangle=0$, then

$$
\operatorname{dim} \operatorname{SI}(Q, \beta)_{\langle\alpha, \cdot,\rangle}=\operatorname{dim} \operatorname{SI}(Q, \alpha)_{-\langle\cdot, \beta\rangle}
$$

Denote this common value of the dimensions of the weight spaces by $\alpha \circ \beta$. . As shown in [DSW07], the number $\alpha \circ \beta$ is the number of subrepresentations of dimension $\alpha$ of a general representation of dimension $\alpha+\beta$. This is calculated either in terms of Schur functors or, equivalently, in terms of Schubert calculus, and is independent of the base field.

By the saturation of effective weights (Theorem 2.26) and the reciprocity property, we have

$$
\alpha \circ \beta \neq 0 \Longleftrightarrow r \alpha \circ s \beta \neq 0, \forall r, s \geqslant 1 \text {. }
$$

We explain later how this statement can be improved (Lemma 2.40) and is conjectured to hold even more stringently (Conjecture 2.41).

Schofield's semi-invariants $c^{V}$ (or $c_{W}$ ) together with his study of general representations provide a description of the set $\Sigma(Q, \beta)$ in the following way. We use the notation $\alpha \hookrightarrow \beta$ to mean that every $\beta$-dimensional representation has a subrepresentation of dimension $\alpha$. With this notation, we can describe the set of integral effective weights $\Sigma(Q, \beta)$ in terms of semi-stability.

Theorem 2.32 ([DW00a], Theorem 3). Let $Q$ be a quiver and $\beta$ a dimension vector. If $\sigma=\langle\alpha, \cdot\rangle \in \mathbb{Z}^{Q_{0}}$ is a weight with $\alpha \in \mathbb{Z}^{Q_{0}}$, then the following statements are equivalent:

1. $\operatorname{dim} \operatorname{SI}(Q, \beta)_{\sigma} \neq 0$, meaning $\sigma \in \Sigma(Q, \beta)$;

2. $\sigma(\beta)=0$ and $\sigma\left(\beta^{\prime}\right) \leqslant 0$ for every $\beta^{\prime} \hookrightarrow \beta$;

3. $\alpha$ is a dimension vector, $\sigma(\beta)=0$, and $\alpha \hookrightarrow \alpha+\beta$; 
4. $\beta$ is $\sigma$-semi-stable.

In particular, for dimension vectors $\alpha$ and $\beta$,

$$
\alpha \circ \beta \neq 0 \Leftrightarrow \beta \text { is }\langle\alpha, \cdot\rangle \text {-semi-stable } \Leftrightarrow \alpha \text { is }-\langle\cdot, \beta\rangle \text {-semi-stable. }
$$

Some of the necessary and sufficient linear homogeneous inequalities above turn out to be redundant. In order to describe a minimal list among these, we need the following result.

Theorem 2.33 ([Sch92], Theorem 6.1). Let $\beta \in \mathbb{Z}_{\geqslant 0}^{Q_{0}}$ be a dimension vector. The following are equivalent:

1. there exists a $\beta$-dimensional representation $V$ such that $\operatorname{End}_{Q}(V, V) \cong \mathbb{C}$;

2. $\sigma_{\beta}\left(\beta^{\prime}\right)<0$ for all $\beta^{\prime} \hookrightarrow \beta, \beta^{\prime} \neq 0, \beta$, where $\sigma_{\beta}=\langle\beta, \cdot\rangle-\langle\cdot, \beta\rangle$.

Any such $\beta$ is called a Schur root and a representation with these properties is a Schur representation.

With this theorem, we can minimally describe the effective weights by semistability conditions.

\subsubsection{The facets of the cone of effective weights}

For a dimension vector $\beta$, let $\mathbb{H}(\beta)=\left\{\sigma \in \mathbb{R}^{Q_{0}} \mid \sigma(\beta)=0\right\}$. Consider the rational convex polyhedral cone

$$
C(Q, \beta)=\left\{\sigma \in \mathbb{H}(\beta) \mid \sigma\left(\beta^{\prime}\right) \leqslant 0 \text { for all } \beta^{\prime} \hookrightarrow \beta\right\}
$$

We call $C(Q, \beta)$ the cone of effective weights associated to $Q$ and $\beta$. Note that $\Sigma(Q, \beta)=C(Q, \beta) \cap \mathbb{Z}^{Q_{0}}$. Like in Theorem 2.32, we will describe an effective weight $\sigma$ in terms of semi-stability. At the same time, we will describe the facets of $C(Q, \beta)$. 
This requires the following decomposition of dimension vectors. We say $V \in \operatorname{rep}(Q, \alpha)$ is a general representation if it lies in a sufficiently small Zariski open subset, where "sufficiently" depends on the context.

Definition 2.34. Let $\alpha$ a dimension vector, and $\sigma$ a weight such that $\sigma(\alpha)=0$. We call

$$
\alpha=\alpha_{1} \dot{+} \ldots \dot{+} \alpha_{s}
$$

the $\sigma$-stable decomposition of $\alpha$ if a general representation of dimension $\alpha$ has a Jordan-Hölder filtration with composition factors of dimension $\alpha_{1}, \ldots, \alpha_{s}$, in some order, including multiplicity.

We may rewrite the $\sigma$-stable decomposition of a dimension vector $\alpha$ by grouping together the common sub-dimension vectors. If $\alpha_{i}$ occurs $c_{i}$ times as the dimension vector of a composition factor in the Jordan-Hölder filtration of $\alpha$, we write the $\sigma$-stable decomposition of $\alpha$ as

$$
\alpha=c_{1} \cdot \alpha_{1} \dot{+} c_{2} \cdot \alpha_{2} \dot{+} \ldots \dot{+} c_{s} \cdot \alpha_{s},
$$

where $c_{i} \in \mathbb{Z}_{>0}$ for all $i$ and $\alpha_{i} \neq \alpha_{j}$ if $i \neq j$.

The following theorem describes the relationship between the facets of the cone $C(Q, \beta)$ and the $\sigma$-stable decomposition. A facet of a polyhedral cone is a face of maximal dimension.

Theorem 2.35 ([DW11], Corollary 5.2). Let $Q$ be a quiver without oriented cycles and $N$ vertices, and $\beta$ a Schur root. Then

1. $\operatorname{dim} C(Q, \beta)=N-1$, and 
2. every facet of the cone $C(Q, \beta)$ is of the form

$$
\mathbb{H}\left(\beta_{1}\right) \bigcap C(Q, \beta)
$$

where $\beta=c_{1} \beta_{1}+c_{2} \beta_{2}$ with $\beta_{1}, \beta_{2}$ Schur roots, $\beta_{1} \circ \beta_{2}=1$, and $c_{i}=1$ whenever $\left\langle\beta_{i}, \beta_{i}\right\rangle<0$

Consequently, $\sigma \in C(Q, \beta)$ if and only if $\sigma(\beta)=0$ and $\sigma\left(\beta_{1}\right) \leqslant 0$ for every decomposition $\beta=c_{1} \beta_{1}+c_{2} \beta_{2}$ with $\beta_{1}, \beta_{2}$ Schur roots, $\beta_{1} \circ \beta_{2}=1$, and $c_{i}=1$ whenever $\left\langle\beta_{i}, \beta_{i}\right\rangle<0$.

The last part of (2) follows from the general description of the facets and Theorem 2.32 .

Remark 2.36. While we could replace $\beta_{1} \circ \beta_{2}=1$ with $\beta_{1} \circ \beta_{2} \neq 0$ in Theorem $2.35(2)$, this would give a longer list of inequalities. The condition $\beta_{1} \circ \beta_{2}=1$ ensures a complete and irredundant list of necessary and sufficient inequalities.

A more precise description of the facets of the cone $C(Q, \beta)$ is given by the following.

Definition 2.37. For a dimension vector $\beta$, define $W_{2}(Q, \beta)$ as the set of all ordered pairs $\left(\beta_{1}, \beta_{2}\right)$ such that:

1. $\beta=c_{1} \beta_{1}+c_{2} \beta_{2}$ for some integers $c_{1}, c_{2} \geqslant 1$;

2. $\beta_{1}$ and $\beta_{2}$ are Schur roots;

3. $s_{1} \beta_{1} \circ s_{2} \beta_{2}=1$ for all $s_{1}, s_{2} \geqslant 1$;

4. $c_{i}=1$ whenever $\left\langle\beta_{i}, \beta_{i}\right\rangle<0$.

Condition (3) above seems to imply infinitely many verifications to check if a decomposition $\left(\beta_{1}, \beta_{2}\right)$ are in $W_{2}(Q, \beta)$. We will later show that it suffices to check 
only finitely many verifications. The following theorem is essential for this. A weight $\sigma$ is called indivisible if the greatest common divisor of its entries is one.

Theorem 2.38 ([DW11], Theorem 3.20, Proposition 3.17). Let $\beta$ be a $\sigma$-semi-stable dimension vector.

1. If $\sigma$ is an indivisible weight and $\beta=c_{1} \cdot \beta_{1} \dot{+} \ldots \dot{+} c_{r} \cdot \beta_{r}$ is the $\sigma$-stable decomposition of $\beta$, then

$$
\operatorname{dim} \operatorname{SI}(Q, \beta)_{s \sigma}=\prod_{i=1}^{r} \operatorname{dim}\left(\operatorname{Sym}^{c_{i}}\left(\operatorname{SI}\left(Q, \beta_{i}\right)_{s \sigma}\right)\right)
$$

for all $s \geqslant 1$.

2. If $\beta=\beta_{1} \dot{+} \ldots \dot{+} \beta_{t}$ is the $\sigma$-stable decomposition of $\beta$ and $p \geqslant 1$, then $p \beta=$ $\left(p \beta_{1}\right)+\ldots \dot{+}\left(p \beta_{t}\right)$ is the $\sigma$-stable decomposition of $p \beta$, where

$$
\left(p \beta_{i}\right)= \begin{cases}\underbrace{\beta_{i}+\ldots \dot{+} \beta_{i}}_{p \text { times }} & \text { if }\left\langle\beta_{i}, \beta_{i}\right\rangle \in\{0,1\} \\ p \beta_{i} & \text { if }\left\langle\beta_{i}, \beta_{i}\right\rangle<0 .\end{cases}
$$

Lemma 2.39. If $\alpha, \beta$ are dimension vectors, then

$$
s_{1} \alpha \circ s_{2} \beta=1 \forall s_{1}, s_{2} \in \mathbb{Z}_{\geqslant 1} \Leftrightarrow s_{1} \alpha \circ \beta=1 \forall s_{1} \in \mathbb{Z}_{\geqslant 1} .
$$

Proof. If $s_{1} \alpha \circ \beta=1$ for all $s_{1} \geqslant 1$, then $\alpha \circ \beta \neq 0$, so $\beta$ is $\sigma$-semi-stable by Theorem 2.32, where $\sigma=\langle\alpha, \cdot\rangle$. Since $\operatorname{dim} \operatorname{SI}(Q, \beta)_{\sigma} \leqslant \operatorname{dim} \operatorname{SI}(Q, \beta)_{r \sigma}$ for all $r \geqslant 1$, suppose $\sigma$ is indivisible. Let

$$
\beta=c_{1} \beta_{1} \dot{+} \ldots \dot{+} c_{t} \beta_{t}
$$

be the $\sigma$-stable decomposition of $\beta$. Then

$$
\operatorname{dim} \operatorname{SI}(Q, \beta)_{s_{1} \sigma}=\prod_{i=1}^{t} \operatorname{dim}\left(\operatorname{Sym}^{c_{i}}\left(\operatorname{SI}\left(Q, \beta_{i}\right)_{s_{1} \sigma}\right)\right)
$$

by Theorem 2.38, and hence $\operatorname{dim} \operatorname{SI}\left(Q, \beta_{i}\right)_{s_{1} \sigma}=1$ for all $s_{1} \geqslant 1$. 
We claim next that each $\beta_{i}$ is a Schur root. Since $\beta_{i}$ is $\sigma$-stable for each $i$, it follows from Theorem 2.32 that $\sigma\left(\beta^{\prime}\right)<0$ for every $\beta^{\prime} \hookrightarrow \beta_{i}, \beta^{\prime} \neq 0, \beta_{i}$. Then for an arbitrary effective weight $\sigma^{\prime} \in C\left(Q, \beta_{i}\right), n \sigma-\sigma^{\prime}$ lies in $C\left(Q, \beta_{i}\right)$ for a large enough $n$, which implies

$$
\operatorname{dim} \operatorname{SI}\left(Q, \beta_{i}\right)_{\sigma^{\prime}} \leqslant \operatorname{dim} \operatorname{SI}\left(Q, \beta_{i}\right)_{n \sigma}
$$

Thus, $\operatorname{dim} \operatorname{SI}\left(Q, \beta_{i}\right)_{\sigma^{\prime}} \leqslant 1$ for any weight $\sigma^{\prime} \in C\left(Q, \beta_{i}\right)$. This is equivalent to there being no nontrivial rational $\operatorname{GL}\left(\beta_{i}\right)$-invariants, and thus, $\operatorname{GL}\left(\beta_{i}\right)$ acts on $\operatorname{Rep}\left(Q, \beta_{i}\right)$ with a dense orbit, implying that $\left\langle\beta_{i}, \beta_{i}\right\rangle=1$. This proves that $\beta_{i}$ is a Schur root, which by Theorem 2.38 also shows that

$$
s_{2} \beta=\left(s_{2} c_{1}\right) \cdot \beta_{1} \dot{+} \ldots\left(s_{2} c_{t}\right) \cdot \beta_{t}
$$

is the $\sigma$-stable decomposition of $s_{2} \beta$. Thus,

$$
\operatorname{dim} \operatorname{SI}\left(Q, s_{2} \beta\right)_{s_{1} \sigma}=\prod_{i=1}^{t} \operatorname{dim}\left(\operatorname{Sym}^{s_{2} c_{i}}\left(\operatorname{SI}\left(Q, \beta_{i}\right)_{s_{1} \sigma}\right)\right)
$$

We then conclude that $\operatorname{dim} \operatorname{SI}\left(Q, s_{2} \beta\right)_{s_{1} \sigma}=1$ for all $s_{1}, s_{2} \geqslant 1$.

In particular, condition (3) in Definition 2.37 may be replaced by $s_{1} \beta_{1} \circ \beta_{2}=$ $1 \forall s_{1} \geqslant 1$. In fact, we can reduce this even further. As proven in [DW02], the stretched function $P(n):=\operatorname{dim}\left(\operatorname{SI}\left(Q, \beta_{2}\right)_{n\left\langle\beta_{1},\right\rangle^{\prime}}\right)$ is in fact a polynomial with rational coefficients. As such, it follows from Lemma 2.39 that we need only check the first $d+1$ terms, where $d$ is the degree of the polynomial $P(n)$. This proves the following.

Lemma 2.40. Let $\alpha, \beta$ be dimension vectors and suppose $\alpha \circ \beta \neq 0$. Then there is an integer d such that

$$
s_{1} \alpha \circ s_{2} \beta=1 \forall s_{1}, s_{2} \geqslant 1 \Leftrightarrow s_{1} \alpha \circ \beta=1 \forall 1 \leqslant s_{1} \leqslant d .
$$


One may obviously ask if this reduces any further. For star quivers (the quivers used in [DW00a] to represent Littlewood-Richardson coefficients as the dimension of a weight space of semi-invariants), we can indeed reduce to the extreme case, which is Fulton's conjecture. Knutson, Tao, and Woodward [KTW04] provided a combinatorial proof of this while Belkale [Bel07] proved it geometrically. In general, however, it remains unproven.

Conjecture 2.41 (Rigidity conjecture). ([DW00b], Conjecture 30) For dimension vectors $\beta_{1}, \beta_{2}$,

$$
s_{1} \beta_{1} \circ s_{2} \beta_{2}=1 \forall s_{1}, s_{2} \geqslant 1 \Leftrightarrow \beta_{1} \circ \beta_{2}=1 .
$$

We can now give a precise description of the facets of the cone $C(Q, \beta)$. In fact, the following theorem is originally stated as a bijection between faces of codimension $r$ and ordered sequences of length $r$, but we won't need this more general statement.

Theorem 2.42 ([DW11], Theorem 5.1). Let $Q$ be a quiver without oriented cycles and $\beta$ a Schur root. Let $\mathcal{F}(Q, \beta)$ denote the set of all facets of $C(Q, \beta)$. Then the map

$$
W_{2}(Q, \beta) \rightarrow \mathcal{F}(Q, \beta)
$$

defined by

$$
\left(\beta_{1}, \beta_{2}\right) \in W_{2}(Q, \beta) \mapsto C\left(Q, \beta_{1}\right) \bigcap C\left(Q, \beta_{2}\right)=\mathbb{H}\left(\beta_{1}\right) \bigcap C(Q, \beta)
$$

is a bijection. Thus, a minimal list of linear homogeneous inequalities defining the cone $C(Q, \beta)$ is obtained by $\sigma(\beta)=0$ and $\sigma\left(\beta_{1}\right) \leqslant 0$ for all $\left(\beta_{1}, \beta_{2}\right) \in W_{2}(Q, \beta)$.

Remark 2.43. If Conjecture 2.41 is true, then the list of inequalities in Theorem 2.35 are the same as those in Theorem 2.42, and hence are minimal. 


\subsubsection{Matrix equations and moment maps}

In order to state the generalized eigenvalue problems for the multiplicities (1.2) and (1.3), two results from linear algebra will be fundamental for us.

King [Kin94] proved that for a quiver without oriented cycles and a weight $\sigma \in \mathbb{Z}^{Q_{0}}$, the set of solutions to a certain set of matrix equations (up to a product of unitary groups) is the symplectic quotient description of a certain moduli space for quivers. A short proof of the following precise description of this may be found in [CG02], Theorem 2.4.

Proposition 2.44. Let $Q$ be a quiver without oriented cycles, $\beta$ a dimension vector, and $\sigma \in \mathbb{R}^{Q_{0}}$. The following are equivalent:

1. $\sigma \in C(Q, \beta) \cap \mathbb{Z}^{Q_{0}}$;

2. there exists $W=\{W(a)\}_{a \in Q_{1}} \in \operatorname{Rep}(\mathrm{Q}, \beta)$ satisfying

$$
\sum_{\substack{a \in Q_{1} \\ t a=x}} W(a)^{*} W(a)-\sum_{\substack{a \in Q_{1} \\ h a=x}} W(a) W(a)^{*}=\sigma(x) \operatorname{Id}_{\beta(x)}
$$

for all $x \in Q_{0}$, where $W(a)^{*}$ is the adjoint of $W(a)$ with respect to the standard Hermitian inner product on $\mathbb{C}^{n}$.

In fact, Proposition 2.44 may be extended to arbitrary points in $C(Q, \beta)$ rather than only ones with integer-valued coordinates; see Section 2 of [Chi06] for a description of the moment map and proof.

The consequence of Proposition 2.44 that we will need in particular is the following. This result will allow us to build up an $n \times n$ Hermitian matrix along each flag of the quivers which will represent the generalized Littlewood-Richardson coefficients. 
Lemma 2.45. Let $\sigma(1), \ldots, \sigma(n-1)$ be non-positive real numbers. The following are equivalent:

1. there exist $W_{i} \in \operatorname{Mat}_{i \times(i+1)}(\mathbb{C}), 1 \leqslant i \leqslant n-1$, such that

$$
\begin{aligned}
W_{i} W_{i}^{*}-W_{i-1}^{*} W_{i-1} & =-\sigma(i) \operatorname{Id}_{i} \quad \text { for } 2 \leqslant i \leqslant n-1, \\
W_{1} W_{1}^{*} & =-\sigma(1) ;
\end{aligned}
$$

2. the matrix $H=W_{n-1}^{*} W_{n-1}$ is Hermitian and has eigenvalues

$$
v(k)=-\sum_{j=k}^{n-1} \sigma(j), \quad \forall 1 \leqslant k \leqslant n-1 \quad \text { and } \quad v(n)=0 .
$$

Proof. See Section 3.4 of [CG02]. 


\section{Chapter 3}

\section{A quiver interpretation of generalized Littlewood-Richardson coefficients}

This chapter includes the main results of this thesis. We begin by defining specific quivers, which we call the sun and generalized star quivers, along with dimension vectors and weights so that multiplicities (1.2) and (1.3) arise as the dimensions of the weight spaces of quiver semi-invariants. This will allow us to prove the saturation of these multiplicities and use quiver invariant theory to study their combinatorial properties. After proving their saturation, we provide Horn-type inequalities which allow us to check if a weight is effective or not through a recursive procedure. Finally, we find the corresponding eigenvalue problems to finish proving statements corresponding to Horn's conjecture for these two multiplicities.

\subsection{Saturation properties}

In this section we will show that each of the multiplicities (1.2) and (1.3) arise as the dimension of the weight space of semi-invariants for a certain quiver and dimension vector which we construct. A proof of the saturation of the multiplicities will then follow from Theorem 2.26. 


\subsubsection{Sun quiver}

Construct a quiver $Q$ in the following way: for $k \geqslant 2$, start with a regular $2 k$-gon with the vertices labeled $(n, i), 1 \leqslant i \leqslant 2 k$, which we call the central vertices, and an arrow connecting $(n, i)$ with $(n, i+1)$ (we will always consider $(n, 2 k+1)=(n, 1))$, where the arrows alternate in direction. At each central vertex $(n, i)$ attach an equioriented $A_{n}$ quiver, called a flag and denoted $\mathscr{F}(i)$, where each $A_{n}$ is directed the same way as the arrows at the central vertex $(n, i)$. We will later associate each flag with a weakly decreasing sequence with at most $n$ parts and each central arrow with some other partition. For instance, for $k=3$ the quiver looks like

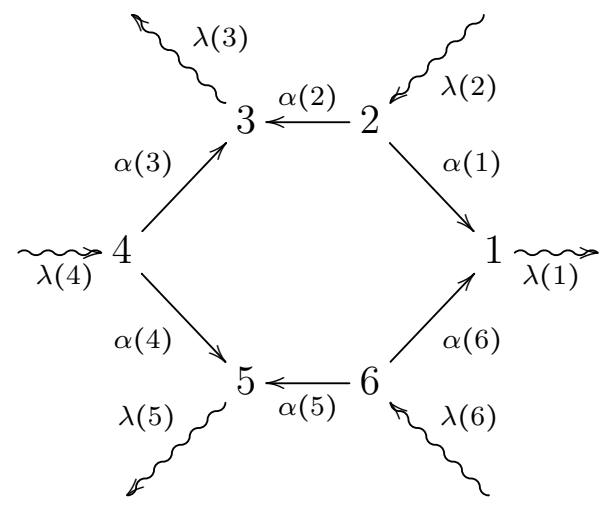

with $n$ vertices along each flag, denoted here by wavy arrows. Label the $j^{\text {th }}$ vertex along the $i^{\text {th }}$ flag by $(j, i)$, numbered so that $(n, i)$ denotes each center vertex or simply $i$ when it is understood. For consistency we'll always have $\mathscr{F}(i)$ going into the central vertex if $i$ is even and out if $i$ is odd.

Define the dimension vector $\beta$ as $\beta(j, i)=j$ for each $1 \leqslant i \leqslant 2 k$ and $1 \leqslant j \leqslant n$. We will show that the generalized Littlewood-Richardson coefficient in (1.2) is the dimension of the weight space of semi-invariants for a certain weight for this quiver and the associated dimension vector $\beta$. We have labeled each flag and central arrow by the 
sequence we will want to eventually associate to it when we calculate the dimension of this particular weight space of semi-invariants. More specifically, we'll associate the weakly decreasing sequence $\lambda(i)$ to flag $\mathscr{F}(i)$ with central arrows $\alpha(i-1), \alpha(i)$ both entering vertex $(n, i)$ when $i$ is odd and leaving when $i$ is even, and $\alpha(j)$ will likewise denote the partition associated to this arrow. Throughout the rest of this subsection we will only consider this quiver, which we call the sun quiver or the $2 k$ sun quiver when we want to emphasize the number of flags, and dimension vector $\beta$ defined by $\beta(j, i)=j$.

Lemma 3.1. Let $\sigma \in \mathbb{Z}^{Q_{0}}$ be a weight for the $2 k$-sun quiver, $k \geqslant 2$. If $\operatorname{dim} \operatorname{SI}(Q, \beta)_{\sigma} \neq$ 0 , then the weight must satisfy $(-1)^{i} \sigma(j, i) \geqslant 0$ for all $1 \leqslant j \leqslant n, 1 \leqslant i \leqslant 2 k$. Furthermore,

$$
\operatorname{dim} \operatorname{SI}(Q, \beta)_{\sigma}=\sum c_{\alpha(1), \alpha(2)}^{\phi(1)} c_{\alpha(2), \alpha(3)}^{\phi(2)} \cdots c_{\alpha(2 k), \alpha(1)}^{\phi(2 k)},
$$

where the sum ranges over all partitions $\alpha(1), \ldots, \alpha(2 k)$, and

$$
\phi(i)=\left(n^{(-1)^{i} \sigma(n, i)}, \ldots, 1^{(-1)^{i} \sigma(1, i)}\right)^{\prime}, \quad 1 \leqslant i \leqslant 2 k .
$$

Proof. Define $V_{j}(i)=\mathbb{C}^{\beta(j, i)}=\mathbb{C}^{j}$ as the vector space assigned to vertex $(j, i)$. To compute the space of semi-invariants, we need an explicit description of the affine coordinate ring of $\operatorname{rep}(Q, \beta)$. For this, denote the $i^{\text {th }}$ flag as $\mathscr{F}(i)$, and define

$$
V_{\mathscr{F}(i)}= \begin{cases}\bigoplus_{j=1}^{n-1} \operatorname{Hom}\left(V_{j+1}(i), V_{j}(i)\right) & i \text { odd } \\ \bigoplus_{j=1}^{n-1} \operatorname{Hom}\left(V_{j}(i), V_{j+1}(i)\right) & i \text { even. }\end{cases}
$$

Then $\operatorname{rep}(Q, \beta)$ decomposes as

$$
\operatorname{rep}(Q, \beta)=\bigoplus_{i=1}^{2 k} V_{\mathscr{F}(i)} \oplus \bigoplus_{i=1}^{k}\left(\operatorname{Hom}\left(V_{n}(2 i), V_{n}(2 i-1)\right) \oplus \operatorname{Hom}\left(V_{n}(2 i), V_{n}(2 i+1)\right)\right)
$$


and the affine coordinate ring is

$$
\mathbb{C}[\operatorname{rep}(Q, \beta)] \cong \bigotimes_{i=1}^{2 k} k\left[V_{\mathscr{F}(i)}\right] \otimes \bigotimes_{i=1}^{k}\left(\mathbb{C}\left[\operatorname{Hom}\left(V_{n}(2 i), V_{n}(2 i-1)\right)\right] \otimes \mathbb{C}\left[\operatorname{Hom}\left(V_{n}(2 i), V_{n}(2 i+1)\right)\right]\right)
$$

We first find the contribution of each flag $\mathscr{F}(i)$ going out of a central vertex, meaning when $i$ is odd. In this case, by using the Cauchy formula (2.15),

$$
\begin{aligned}
k\left[V_{\mathscr{F}(i)}\right] & =\bigotimes_{j=1}^{n-1} k\left[V_{j+1}^{*}(i) \otimes V_{j}(i)\right] \\
& =\bigotimes_{j=1}^{n-1} S\left(V_{j+1}(i) \otimes V_{j}(i)^{*}\right) \\
& \cong \bigoplus_{\phi^{1}(i), \ldots, \phi^{n-1}(i)} S^{\phi^{1}(i)} V_{1}(i)^{*} \otimes \bigotimes_{j=2}^{n-1}\left(S^{\phi^{j-1}(i)} V_{j}(i) \otimes S^{\phi^{j}(i)} V_{j}(i)^{*}\right) \otimes S^{\phi^{n-1}(i)} V_{n}(i) .
\end{aligned}
$$

Notice that all the terms except $S^{\phi^{n-1}}(i) V_{n}(i)$ represent the contribution of the first $n-1$ vertices of $\mathscr{F}(i)$. Because the $j^{\text {th }}$ term of $\operatorname{SL}(\beta)$ acts trivially on each $S^{\phi^{m}(i)} V_{k}(i)$ whenever $j \neq k$, the semi-invariants for this affine coordinate ring are precisely the sums of the tensor products of the semi-invariants of each term. That is, the terms of $\mathrm{SL}(\beta)$ distribute to the corresponding terms of the sum across the tensor products.

A summand of this sum is nonzero precisely when the $\phi^{j}(i)$ are such that the semiinvariants of each term in the tensor product is nonzero. Now $\left(S^{\phi^{1}(i)} V_{1}(i)^{*}\right)^{\operatorname{SL}\left(V_{1}(i)\right)} \neq$ 0 if and only if $\phi^{1}(i)$ is of size $w \times \operatorname{dim} V_{1}(i)=w \times 1$ for some $w \in \mathbb{Z}_{\geqslant 0}$ (see Proposition 2.12). In this case, the space is one-dimensional and is spanned by a semi-invariant of weight $-w$. Therefore, $\left(S^{\phi^{1}(i)} V_{1}(i)^{*}\right)^{\mathrm{SL}\left(V_{1}(i)\right)}$ contains a (nonzero) semi-invariant of weight $\sigma(1, i)$ if and only if $\sigma(1, i)<0$ and $\phi^{1}(i)$ is of size $-\sigma(1, i) \times 1=\left(1^{-\sigma(1, i)}\right)^{\prime}$. We know from this that $\left(S^{\phi^{1}(i)} V_{1}(i)^{*}\right)^{\mathrm{SL}\left(V_{1}(i)\right)}$ is nonzero if and only if it is onedimensional.

Next, $\left(S^{\phi^{1}(i)} V_{2}(i) \otimes S^{\phi^{2}(i)} V_{2}(i)^{*}\right)^{\mathrm{SL}\left(V_{2}(i)\right)}$ is nonzero if and only if $\phi^{2}(i)_{p}-\phi^{1}(i)_{p}=$ $k \in \mathbb{Z}$ for all $p$ (Proposition 2.13). That is, $\phi^{2}(i)$ is $\phi^{1}(i)$ plus some extra columns, 
which must be of length $\operatorname{dim} V_{2}(i)=\beta(i, 2)=2$. In this case, the space being nonzero is equivalent to it being spanned by a semi-invariant of weight equal to the negative of the number of extra columns. Hence, $\left(S^{\phi^{1}(i)} V_{2}(i) \otimes S^{\phi^{2}(i)} V_{2}(i)^{*}\right)^{\mathrm{SL}\left(V_{2}(i)\right)}$ contains a semi-invariant of weight $\sigma(2, i)$ if and only if the space is one-dimensional and $\phi^{2}(i)=\left(2^{-\sigma(2, i)}, 1^{-\sigma(1, i)}\right)^{\prime}$.

Reasoning this way and continuing by sorting the spaces for semi-invariants in $\operatorname{SI}(Q, \beta)$ of weight $\sigma$, we have that $\phi^{1}(i)$ is of size $-\sigma(1, i) \times 1$ and $\phi^{j}(i)$ is obtained from $\phi^{j-1}(i)$ by adjoining a rectangle of size $-\sigma(j, i) \times j$ to the left of it. Thus, $\phi^{n-1}(i)=((n-$ $\left.1)^{-\sigma(n-1, i)}, \ldots, 1^{-\sigma(1, i)}\right)^{\prime}$ and the contribution of the flag $\mathscr{F}(i)$ for odd $i$ to $\operatorname{SI}(Q, \beta)_{\sigma}$ is precisely $S^{\phi^{n-1}(i)} V_{n}(i)$ since the other nonzero terms are all of dimension one.

Similarly, if $\mathscr{F}(i)$ is a flag going into a central vertex, meaning $i$ is even, then $\sigma(j, i) \geqslant 0$ for all $1 \leqslant j \leqslant n-1$, and the contribution of the flag $\mathscr{F}(i)$ to $\operatorname{SI}(Q, \beta)_{\sigma}$ is $S^{\phi^{n-1}(i)} V_{n}(i)^{*}$ with

$$
\phi^{n-1}(i)=\left((n-1)^{\sigma(n-1, i)}, \ldots, 1^{\sigma(1, i)}\right)^{\prime} .
$$

In addition, by using Cauchy's formula again and labeling the central arrows as $a_{i}$, we have

$$
\bigotimes_{i=1}^{2 k} \mathbb{C}\left[\operatorname{Hom}\left(V_{n}\left(t a_{i}\right), V_{n}\left(h a_{i}\right)\right]=\bigoplus \bigotimes_{i=1}^{2 k} S^{\alpha(i)} V_{n}\left(t a_{i}\right) \otimes S^{\alpha(i)} V_{n}^{*}\left(h a_{i}\right),\right.
$$

where the sum is over all partitions $\alpha(i)$ with at most $n$ parts. By taking into account the weights at the central vertices and denoting $V_{n}(i)$ as simply $V(i)$, the central vertices produce the following spaces of semi-invariants:

$$
\begin{array}{r}
S^{\phi^{n-1}(2 i-1)} V(2 i-1) \otimes S^{\alpha(2 i-2)} V(2 i-1)^{*} \otimes S^{\alpha(2 i-1)} V(2 i-1)^{*}, \\
S^{\phi^{n-1}(2 i)} V(2 i)^{*} \otimes S^{\alpha(2 i)} V(2 i) \otimes S^{\alpha(2 i-1)} V(2 i)
\end{array}
$$


for each $i=1, \ldots, k$. Putting these together, the space of semi-invariants $\operatorname{SI}(Q, \beta)_{\sigma}$ is isomorphic to

$$
\begin{aligned}
\bigoplus & \bigotimes_{i=1}^{k}\left(S^{\phi^{n-1}(2 i-1)} V(2 i-1) \otimes S^{\alpha(2 i-2)} V(2 i-1)^{*} \otimes S^{\alpha(2 i-1)} V(2 i-1)^{*}\right)^{\mathrm{SL}(V(2 i-1))} \otimes \\
& \bigotimes_{i=1}^{k}\left(S^{\phi^{n-1}(2 i)} V(2 i)^{*} \otimes S^{\alpha(2 i)} V(2 i) \otimes S^{\alpha(2 i-1)} V(2 i)\right)^{\mathrm{SL}(V(2 i))},
\end{aligned}
$$

where the sum is over all partitions $\alpha(1), \ldots, \alpha(2 k)$ with at most $n$ parts. By tensoring with appropriate powers of the determinant to obtain $\operatorname{GL}(\beta)$-representations, the dimension of the space of semi-invariants will be precisely as claimed. More specifically,

$$
\begin{aligned}
& \operatorname{dim}\left(\left(S^{\phi^{n-1}(j)} V(j) \otimes S^{\alpha(j-1)} V(j)^{*} \otimes S^{\alpha(j)} V(j)^{*} \otimes \operatorname{det}_{V(j)}^{-\sigma(n, j)}\right)^{\mathrm{GL}(V(j))}\right)=c_{\alpha(j-1), \alpha(j)}^{\phi(j)}, \quad j \text { odd } \\
& \operatorname{dim}\left(\left(S^{\phi^{n-1}(j)} V(j)^{*} \otimes S^{\alpha(j)} V(j) \otimes S^{\alpha(j-1)} V(j) \otimes \operatorname{det}_{V(j)}^{-\sigma(n, j)}\right)^{\mathrm{GL}(V(j))}\right)=c_{\alpha(j-1), \alpha(j)}^{\phi(j)}, \quad j \text { even }
\end{aligned}
$$

for each $j=1, \ldots, 2 k$. The dimension of $\operatorname{SI}(Q, \beta)_{\sigma}$ is then the sum over all partitions $\alpha(1), \ldots, \alpha(2 k)$ with at most $n$ parts of products of these Littlewood-Richardson coefficients.

For weakly decreasing sequences $\lambda(1), \ldots, \lambda(2 k)$ of $n$ integers, define the weight $\sigma_{1}$ as

$$
\sigma_{1}(j, i)= \begin{cases}(-1)^{i}\left(\lambda(i)_{j}-\lambda(i)_{j+1}\right) & 1 \leqslant i \leqslant 2 k, 1 \leqslant j \leqslant n-1 \\ (-1)^{i} \lambda(i)_{n} & 1 \leqslant i \leqslant 2 k, j=n .\end{cases}
$$

The following is immediate from calculating what the $\phi(i)$ are with respect to this weight.

Lemma 3.2. Let $\lambda(1), \ldots, \lambda(2 k), k \geqslant 2$, be weakly decreasing sequences of $n$ integers. Then for every integer $r \geqslant 1$, we have

$$
f_{1}(r \lambda(1), \ldots, r \lambda(2 k))=\sum c_{\alpha(1), \alpha(2)}^{r \lambda(1)} c_{\alpha(2), \alpha(3)}^{r \lambda(2)} \cdots c_{\alpha(2 k), \alpha(1)}^{r \lambda(2 k)}=\operatorname{dim} \operatorname{SI}(Q, \beta)_{r \sigma_{1}} .
$$


In particular, when $k=3$ and $r=1$ the dimension of this weight space of semiinvariants is the multiplicity of the branching rule for the diagonal embedding of $\operatorname{GL}(n)$.

Proof. Because the general case is proven in precisely the same way, assume $r=1$. The proof is then the same as that of Lemma 3.1 because $\phi(i)=\lambda(i)$ with this weight.

Remark 3.3. While it is clear that $\lambda(1), \ldots, \lambda(2 k)$ must be partitions if $f_{1}(\lambda(1), \ldots, \lambda(2 k))$ is to be nonzero, this is verified from the conditions for $\sigma$ in Lemma 3.1 and the description of $\sigma_{1}$.

Proof of Theorem 1.7. By representing the multiplicity as the dimension of a weight space of semi-invariants as in Lemma 3.2, the saturation of this multiplicity immediately follows from Theorem 2.26.

Remark 3.4. In this way, we have written the generalized Littlewood-Richardson coefficient $f_{1}(\lambda(1), \ldots, \lambda(m))$ as the dimension of a certain weight space of semiinvariants of some quiver. However, we can only express the generalized LittlewoodRichardson coefficient in terms of quiver invariant theory when $m$ is even and at least four. When $m \geqslant 3$ is odd, this process fails because the first and last flags will be oriented the same direction which would require the central arrow connecting the first and last central vertices to be pointed both directions, an impossibility, while if $m=2$ we would have an oriented cycle. 


\subsubsection{Generalized star quiver}

The same type of analysis can be applied to represent multiplicity (1.3) as the dimension of a weight space of semi-invariants. Doing so, however, depends on the parity of $m$. Consider the quivers

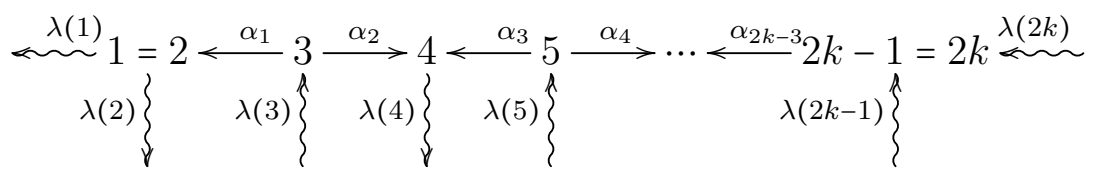

and

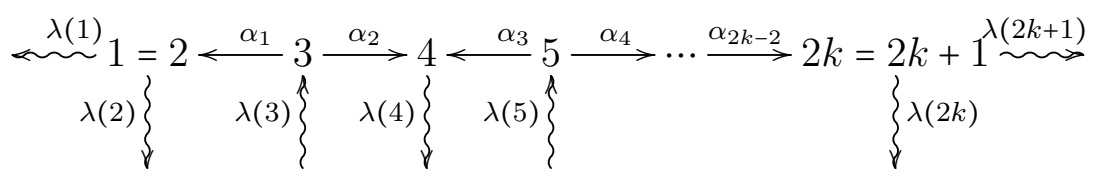

Here, we've identified the central vertex connecting the first two flags and likewise for the last two flags. Notice that this is precisely the quiver in [Chi08], except that the flags on the ends are not alternating in direction. Again, define $\beta(j, i)=$ j. Throughout this thesis, we call either of these quivers the generalized $m$-star quivers, where $m$ is the number of flags, or simply the generalized star quiver. We only consider the cases where $m \geqslant 4$.

Lemma 3.5. Let $\sigma \in \mathbb{Z}^{Q_{0}}$ be a weight for the generalized $m$-star quiver and suppose $k \geqslant 2$. If $m=2 k$ and $\operatorname{dim} \operatorname{SI}(Q, \beta)_{\sigma} \neq 0$, then the weight must satisfy for each $1 \leqslant j \leqslant n$,

$$
(-1)^{i+1} \sigma(j, i) \geqslant 0 \quad 2 \leqslant i \leqslant 2 k-1, \quad \sigma(j, 1) \leqslant 0, \quad \sigma(j, 2 k) \geqslant 0
$$

while if $m=2 k+1$,

$$
(-1)^{i+1} \sigma(j, i) \geqslant 0 \quad 2 \leqslant i \leqslant 2 k, \quad \sigma(j, 1) \leqslant 0, \quad \sigma(j, 2 k+1) \leqslant 0 .
$$

Moreover, in either case,

$$
\operatorname{dim} \operatorname{SI}(Q, \beta)_{\sigma}=\sum c_{\phi(1), \phi(2)}^{\alpha(1)} c_{\alpha(1), \alpha(2)}^{\phi(3)} c_{\alpha(2), \alpha(3)}^{\phi(4)} \cdots c_{\alpha(m-4), \alpha(m-3)}^{\phi(m-2)} c_{\phi(m-1), \phi(m)}^{\alpha(m-3)},
$$


where

$$
\phi(i)=\left((n-1)^{|\sigma(n-1, i)|}, \ldots, 1^{|\sigma(1, i)|}\right)^{\prime}
$$

and the sum ranges over all partitions $\alpha(1), \ldots, \alpha(m-3)$ with at most $n$ parts.

Proof. The calculation of the contributions of the first $n-1$ vertices along each flag are the same as in Lemma 3.1 precisely because each flag is still equioriented $A_{n}$. Taking into account the central vertices and denoting $V_{n}(i)$ as simply $V(i)$, the central vertices contribute the following spaces of semi-invariants:

$$
\begin{array}{cr}
S^{\phi(1)} V(2) \otimes S^{\phi(2)} V(2) \otimes S^{\alpha(1)} V(2)^{*}, & i=1 \\
S^{\phi(2 i-1)} V(2 i-1)^{*} \otimes S^{\alpha(2 i-3)} V(2 i-1) \otimes S^{\alpha(2 i-2)} V(2 i-1), & 2 \leqslant i \leqslant k-1 \text { if } m=2 k \\
S^{\phi(2 i-1)} V(2 i-1)^{*} \otimes S^{\alpha(2 i-3)} V(2 i-1) \otimes S^{\alpha(2 i-2)} V(2 i-1), & 2 \leqslant i \leqslant k \text { if } m=2 k+1 \\
S^{\phi(2 i)} V(2 i) \otimes S^{\alpha(2 i-2)} V(2 i)^{*} \otimes S^{\alpha(2 i-1)} V(2 i)^{*}, & 2 \leqslant i \leqslant k-1 \text { if } m=2 k \\
S^{\phi(2 i)} V(2 i) \otimes S^{\alpha(2 i-2)} V(2 i)^{*} \otimes S^{\alpha(2 i-1)} V(2 i)^{*}, & 2 \leqslant i \leqslant k \text { if } m=2 k+1 \\
S^{\phi(2 k-1)} V(2 k-1)^{*} \otimes S^{\phi(2 k)} V(2 k-1)^{*} \otimes S^{\alpha(2 k-3)} V(2 k-1), & \text { if } m=2 k \\
S^{\phi(2 k)} V(2 k) \otimes S^{\phi(2 k+1)} V(2 k) \otimes S^{\alpha(2 k-2)} V(2 k)^{*}, & \text { if } m=2 k+1 .
\end{array}
$$

Thus, if $m=2 k$ the space of semi-invariants $\operatorname{SI}(Q, \beta)_{\sigma}$ is isomorphic to

$$
\begin{aligned}
\bigoplus & \left(S^{\phi(1)} V(2) \otimes S^{\phi(2)} V(2) \otimes S^{\alpha(1)} V(2)^{*}\right)^{\mathrm{SL}(V(2))} \otimes \\
& \bigotimes_{i=2}^{k-1}\left(S^{\phi(2 i-1)} V(2 i-1)^{*} \otimes S^{\alpha(2 i-3)} V(2 i-1) \otimes S^{\alpha(2 i-2)} V(2 i-1)\right)^{\mathrm{SL}(V(2 i-1))} \otimes \\
& \bigotimes_{i=2}^{k-1}\left(S^{\phi(2 i)} V(2 i) \otimes S^{\alpha(2 i-2)} V(2 i)^{*} \otimes S^{\alpha(2 i-1)} V(2 i)^{*}\right)^{\mathrm{SL}(V(2 i))} \otimes \\
& \left(S^{\phi(2 k-1)} V(2 k-1)^{*} \otimes S^{\phi(2 k)} V(2 k-1)^{*} \otimes S^{\alpha(2 k-3)} V(2 k-1)\right)^{\mathrm{SL}(V(2 k-1)},
\end{aligned}
$$

while if $m=2 k+1$ it is isomorphic to 


$$
\begin{aligned}
\bigoplus & \left(S^{\phi(1)} V(2) \otimes S^{\phi(2)} V(2) \otimes S^{\alpha(1)} V(2)^{*}\right)^{\mathrm{SL}(V(2))} \otimes \\
& \bigotimes_{i=2}^{k}\left(S^{\phi(2 i-1)} V(2 i-1)^{*} \otimes S^{\alpha(2 i-3)} V(2 i-1) \otimes S^{\alpha(2 i-2)} V(2 i-1)\right)^{\mathrm{SL}(V(2 i-1))} \otimes \\
& \bigotimes_{i=2}^{k}\left(S^{\phi(2 i)} V(2 i) \otimes S^{\alpha(2 i-2)} V(2 i)^{*} \otimes S^{\alpha(2 i-1)} V(2 i)^{*}\right)^{\mathrm{SL}(V(2 i))} \otimes \\
& \left(S^{\phi(2 k)} V(2 k)^{*} \otimes S^{\phi(2 k+1)} V(2 k)^{*} \otimes S^{\alpha(2 k-2)} V(2 k)\right)^{\mathrm{SL}(V(2 k)} .
\end{aligned}
$$

In either case, the dimension is of the form claimed.

Suppose $\lambda(1), \ldots, \lambda(m)$ are weakly decreasing sequences of $n$ integers. If $m=2 k$ define the weight $\sigma_{2}$ by

$$
\sigma_{2}(j, i)= \begin{cases}(-1)^{i+1}\left(\lambda_{j+1}(i)-\lambda_{j}(i)\right) & 1 \leqslant j \leqslant n-1,2 \leqslant i \leqslant 2 k-1 \\ \lambda_{j+1}(i)-\lambda_{j}(i) & 1 \leqslant j \leqslant n-1, i=1 \\ \lambda_{j}(i)-\lambda_{j+1}(i) & 1 \leqslant j \leqslant n-1, i=2 k \\ -\lambda_{n}(1)-\lambda_{n}(2) & i \in\{1,2\}, j=n \\ (-1)^{i+1} \lambda_{n}(i) & 3 \leqslant i \leqslant 2 k-2, j=n \\ \lambda_{n}(2 k-1)+\lambda_{n}(2 k) & i \in\{2 k-1,2 k\}, j=n\end{cases}
$$

while if $m=2 k+1$, define the weight $\sigma_{2^{\prime}}$ by

$$
\sigma_{2^{\prime}}(j, i)= \begin{cases}(-1)^{i+1}\left(\lambda_{j+1}(i)-\lambda_{j}(i)\right) & 1 \leqslant j \leqslant n-1,2 \leqslant i \leqslant 2 k \\ \lambda_{j+1}(i)-\lambda_{j}(i) & 1 \leqslant j \leqslant n-1, i \in\{1,2 k+1\} \\ -\lambda_{n}(1)-\lambda_{n}(2) & i \in\{1,2\}, j=n \\ (-1)^{i+1} \lambda_{n}(i) & 3 \leqslant i \leqslant 2 k-2, j=n \\ -\lambda_{n}(2 k-1)-\lambda_{n}(2 k) & i \in\{2 k, 2 k+1\}, j=n\end{cases}
$$

We then obtain the following results just as in the case for the sun quiver.

Lemma 3.6. With the above notation, for every integer $r \geqslant 1$, we have

$$
\begin{aligned}
f_{2}(r \lambda(1), \ldots, r \lambda(m)) & =\sum c_{\lambda(1), \lambda(2)}^{\alpha(1)} c_{\alpha(1), \alpha(2)}^{\lambda(3)} c_{\alpha(2), \alpha(3)}^{\lambda(4)} \cdots c_{\alpha(m-4), \alpha(m-3)}^{\lambda(m-2)} c_{\lambda(m-1)), \lambda(m)}^{\alpha(m-3)} \\
& =\operatorname{dim} \operatorname{SI}(Q, \beta)_{r \sigma_{2}} .
\end{aligned}
$$

In particular, when $m=6$ and $r=1$ this is the branching multiplicity for the direct sum embedding of $\mathrm{GL}(n)$. 
Theorem 3.7 (Saturation property). Let $\lambda(1), \ldots, \lambda(m)$ be weakly decreasing sequences of $n$ integers. For every integer $r \geqslant 1$,

$$
f_{2}(r \lambda(1), \ldots, r \lambda(m)) \neq 0 \quad \text { if and only if } \quad f_{2}(\lambda(1), \ldots, \lambda(m)) \neq 0 .
$$

Proof. This is immediate from Lemma 3.6 along with Theorem 2.26.

Remark 3.8. Like for the sun quiver, the conditions on an effective weight imply that the $\lambda(i)$ are in fact partitions. Also, the results in this subsection remain true in the case $m=3$ because in this case there is only one central vertex and everything reduces to the star quiver described in [DW00a].

Remark 3.9. The saturation of multiplicity (1.4) is Theorem 1.4 of [Chi08].

\subsection{The facets of the cones of effective weights}

We use the techniques from Section 2.2.4 to find necessary and sufficient Horn-type inequalities for determining if (1.2) or (1.3) is nonzero. Throughout this section, $\beta$ is the dimension vector as previously defined for either of the quivers under consideration.

In order to use the results in the previous section to describe the facets of $C(Q, \beta)$ for the sun quiver and generalized star quiver $Q$, we'll first show that the dimension vector $\beta$ is Schur in either case and determine conditions on the $\beta_{1}^{\prime} \mathrm{s}$ that can appear in the decompositions.

Lemma 3.10. For either the sun quiver or the generalized star quiver, the respective dimension vector $\beta$ is Schur.

Proof. The dimension vector $\beta$ is indivisible, meaning the greatest common divisor of its coordinates is one. By a result of Kac ([Kac82], Theorem $\mathrm{B}(\mathrm{d})$ ), to show $\beta$ is 
Schur, it suffices to show that $\beta$ is in the fundamental region of the graph, meaning that the support of $\beta$ is a connected graph and $\tau_{i}(\beta) \leqslant 0$ for all vertices $i \in Q_{0}$, where $e_{i}$ denotes the dimension vector of the simple representation at vertex $i$ and $\tau_{i}(\cdot):=\left\langle e_{i}, \cdot\right\rangle+\left\langle\cdot, e_{i}\right\rangle$. This is immediately checked to hold for all $n \geqslant 1$ for the sun quiver and $n \geqslant 2$ for the generalized star quiver. For $n=1$, the generalized star quiver is simply $A_{m}$, so $\beta$ is a real Schur root.

Corollary 3.11. Let $Q_{1}$ be the sun quiver and $Q_{2}$ the generalized star quiver. Then

1. $\operatorname{dim} C\left(Q_{1}, \beta\right)=m n-1$, and

2. $\operatorname{dim} C\left(Q_{2}, \beta\right)=m n-3$.

Proof. This immediately follows from Lemma 3.10 and Theorem 2.35.

For either of the two quivers, consider the following dimension vectors $\beta_{1}$, where $e_{(j, i)}$ denotes the dimension vector of the simple representation at vertex $(j, i)$ :

1. $\beta_{1}=e_{(j, i)}$ for a flag $i$ going out of the central vertex, or $\beta_{1}=\beta-e_{(j, i)}$ for a flag $i$ going into a central vertex;

2. $\beta_{1} \neq \beta, \beta_{1} \circ\left(\beta-\beta_{1}\right)=1$, and $\beta_{1}$ is weakly increasing with jumps of at most one along each of the $m$ flags.

Denote the set of such $\beta_{1}$ by $\mathcal{D}$. We show in the next lemma that each $\beta_{1} \in \mathcal{D}$ defines a facet of $C(Q, \beta)$, which is called a regular facet if $\beta_{1}$ is in the form described in (2), while a facet defined by some $\beta_{1}$ as described in (1) is called trivial. The interpretation of the inequalities arising from the $\beta_{1} \in \mathcal{D}$ is given in Remark 3.14.

Lemma 3.12. For either the sun quiver or generalized star quiver $Q$, the regular facets of $C(Q, \beta)$ are of the form

$$
\mathbb{H}\left(\beta_{1}\right) \bigcap C(Q, \beta)
$$


where $\beta_{1}$ is weakly increasing with jumps of at most one along the flags, $\beta_{1} \neq \beta$, and $\beta_{1} \circ\left(\beta-\beta_{1}\right)=1$.

Proof. By Theorem 2.42, a facet $\mathcal{F}$ of the associated cone is of the form

$$
\mathbb{H}\left(\beta_{1}\right) \bigcap C(Q, \beta),
$$

where $\beta_{1}, \beta_{2}$ are Schur roots, $\beta_{1} \circ \beta_{2}=1$, and $\beta=c_{1} \beta_{1}+c_{2} \beta_{2}$ for some $c_{1}, c_{2} \geqslant 1$.

Suppose that $\beta_{1}$ is not simple, meaning the corresponding facet is not trivial. We'll show that $\beta_{1}$ is weakly increasing with jumps of at most one along the flags. Denote $c_{1} \beta_{1}=\beta_{1}^{\prime}$ and $c_{2} \beta_{2}=\beta_{2}^{\prime}$. Because it is clear that $s_{1} \beta_{1} \circ s_{2} \beta_{2} \geqslant \beta_{1} \circ \beta_{2}$ for all $s_{1}, s_{2} \geqslant 1, \beta_{1}^{\prime} \circ$ $\beta_{2}^{\prime} \neq 0$. By Theorem 2.32, it follows that any representation of dimension vector $\beta$ has a subrepresentation of dimension vector $\beta_{1}^{\prime}$. Choose a $\beta$-dimensional representation which is injective along the flags going into a central vertex and surjective along the flags going out of the central vertex. Then $\beta_{1}^{\prime}$ is weakly increasing along the flags going in and has jumps of at most one (from the end of the flag towards the center vertex) along the flags going out, or else the maps couldn't be surjective.

We'll show that $\beta_{1}^{\prime}$ is weakly increasing along each flag $\mathcal{F}(i)$ going out of a central vertex. Suppose to the contrary that $\beta_{1}^{\prime}(l+1)-\beta_{1}^{\prime}(l)<0$ for some $l \in\{1, \ldots, n-1\}$. Then $\beta_{1}^{\prime}-e_{l} \hookrightarrow \beta_{1}^{\prime}$. Moreover, $\beta_{1}^{\prime} \circ \beta_{2}^{\prime} \neq 0$ is equivalent to $\beta_{1}^{\prime}$ being $-\left\langle\cdot, \beta_{2}^{\prime}\right\rangle$-semi-stable by reciprocity (Theorem 2.31). Thus, $\left\langle\beta_{1}^{\prime}-e_{l}, \beta_{2}^{\prime}\right\rangle \geqslant 0$, so $\beta_{2}^{\prime}(l) \leqslant \beta_{2}^{\prime}(l-1)$, implying $\beta_{1}^{\prime}(l) \geqslant 1+\beta_{1}^{\prime}(l-1)$. As we previously showed that $\beta_{1}^{\prime}$ has jumps of at most one along such a flag, we must have $\beta_{1}^{\prime}(l)=1+\beta_{1}^{\prime}(l-1)$. Thus, $c_{1}=1$ and $e_{l} \hookrightarrow \beta_{1}^{\prime}$. We then have that $\beta_{1}^{\prime}=\beta_{1}$ is a Schur root by assumption, hence is $\sigma_{\beta_{1}^{\prime}}$-semistable by Theorem 2.33, and $e_{l}, \beta_{1}^{\prime}-e_{l} \hookrightarrow \beta_{1}^{\prime}$, with $\beta_{1}^{\prime} \neq e_{l}$. Therefore, by the same theorem, $\sigma_{\beta_{1}^{\prime}}\left(e_{l}\right)<0$ and $\sigma_{\beta_{1}^{\prime}}\left(\beta_{1}^{\prime}-e_{l}\right)<0$, which is a contradiction. Thus, $\beta_{1}^{\prime}$ must be weakly increasing along 
the flags going out of a central vertex. By a similar argument, $\beta_{1}^{\prime}$ will have jumps of at most one along each flag going in.

Finally, we'll show that $c_{1}=c_{2}=1$. Because $\beta_{1}^{\prime}=c_{1} \beta_{1}$ has jumps of at most one along each flag, we have $0 \leqslant c_{1}\left(\beta_{1}(l+1, i)-\beta_{1}(l, i)\right) \leqslant 1$ for all $l \in\{1, \ldots, n-1\}$ and $i \in\{1, \ldots, m\}$. If there are no $l, i$ such that $\beta_{1}(l+1, i)-\beta_{1}(l, i) \neq 0$, then $c_{1}=1$, while otherwise there must exist an $i$ such that $\beta_{1}^{\prime}(l, i)=1$, so $c_{1}=1$. Similarly, $c_{2}=1$.

Thus, $\beta=\beta_{1}+\beta_{2}$ with $\beta_{1}$ weakly increasing of jumps of at most one along the flags.

Lemma 3.13. Let $\sigma \in \mathbb{H}(\beta)$ for the sun quiver $Q$. Then $\sigma \in C(Q, \beta)$ if and only if the following are true:

1. $(-1)^{i} \sigma\left(e_{(j, i)}\right) \geqslant 0$ for all $1 \leqslant j \leqslant n-1,1 \leqslant i \leqslant m$;

2. $\sigma\left(\beta_{1}\right) \leqslant 0$ for every $\beta_{1} \neq \beta$ weakly increasing with jumps of at most one along the flags and $\beta_{1} \circ\left(\beta-\beta_{1}\right)=1$.

Proof. The description of the regular facets in Lemma 3.12 proves one direction, while if $\sigma \in C(Q, \beta)$, then $\sigma\left(\beta_{1}\right) \leqslant 0$ for every $\beta_{1} \in \mathcal{D}$ by Theorem 2.32, which is equivalent to $(1)$ and $(2)$.

Remark 3.14. Let $\sigma_{1}$ be the weight we defined for the sun quiver in Equation (3.1). In particular,

$$
\sigma_{1}\left(e_{(j, i)}\right)=(-1)^{i}\left(\lambda(i)_{j}-\lambda(i)_{j+1}\right), \quad 1 \leqslant i \leqslant m, 1 \leqslant j \leqslant n-1 .
$$

The inequalities arising from a trivial facet of $C(Q, \beta)$, as described in (1) in the preceding lemma, called the chamber inequalities, simply state that the sequences $\lambda(i)$ are weakly decreasing sequences of real numbers. Because we will always assume 
this, we exclude these $\beta_{1}$ from our considerations. The inequalities arising from (2) in the lemma are called the regular inequalities, and the corresponding facet is regular.

We can similarly describe the chamber and regular inequalities for the generalized star quiver, which will have the same interpretation. The following is proved exactly the same as Lemma 3.13.

Lemma 3.15. Let $\sigma \in \mathbb{H}(\beta)$ for the generalized star quiver $Q$. Then $\sigma \in C(Q, \beta)$ if and only if the following are true:

1. for all $1 \leqslant j \leqslant n-1$,

$$
\begin{aligned}
& (-1)^{i+1} \sigma(j, i) \geqslant 0, \quad \sigma(j, 1) \leqslant 0, \quad \sigma(j, 2 k) \geqslant 0 \quad \text { if } m=2 k, 2 \leqslant i \leqslant 2 k-1 \\
& (-1)^{i+1} \sigma(j, i) \geqslant 0, \quad \sigma(j, 1) \leqslant 0, \quad \sigma(j, 2 k+1) \leqslant 0 \quad \text { if } m=2 k+1,2 \leqslant i \leqslant 2 k,
\end{aligned}
$$

2. $\sigma\left(\beta_{1}\right) \leqslant 0$ for every $\beta_{1} \neq \beta$ weakly increasing with jumps of at most one along the flags and $\beta_{1} \circ\left(\beta-\beta_{1}\right)=1$.

\subsection{Horn-type inequalities}

Using Lemmas 3.1 and 3.5, we will give closed forms of the inequalities which provide necessary and sufficient conditions for the nonvanishing of the generalized LittlewoodRichardson coefficients.

\subsubsection{Sun quiver}

In this subsection $Q$ denotes the sun quiver. Throughout, let $\beta_{1}$ be a dimension vector which is weakly increasing with jumps of at most one along each of the flags towards 
the central vertices. Define the following jump sets:

$$
I_{i}=\left\{l \mid \beta_{1}(l, i)>\beta_{1}(l-1, i), 1 \leqslant l \leqslant n\right\}
$$

with the convention $\beta_{1}(0, i)=0$ for all $i$. Because $\beta_{1}$ defines a tuple $I=\left(I_{1}, \ldots, I_{m}\right)$, we'll commonly denote $\beta_{1}$ by $\beta_{I}$. Note that $\left|I_{i}\right|=\beta_{I}(n, i)$ for each $i$.

Conversely, each tuple $I=\left(I_{1}, \ldots, I_{m}\right)$ of subsets of $\{1, \ldots, n\}$ defines a dimension vector $\beta_{I}$ because if

$$
I_{i}=\left\{z_{1}(i)<\cdots<z_{r}(i)\right\},
$$

then $\beta_{I}(j, i)=j-1$ for all $z_{k-1}(i) \leqslant j<z_{k}(i)$ for all $1 \leqslant k \leqslant r+1$, with the convention that $z_{0}(i)=0$ and $z_{r+1}(i)=n+1$ for all $i$. This means that going towards the center vertex on the $i^{\text {th }}$ flag, the dimension at a vertex is 0 until the vertex $z_{1}(i)$, at which it becomes 1 and continues to be 1 until the vertex $z_{2}(i)$, at which point it becomes 2 , and so on.

Definition 3.16. For the dimension vector $\beta$ associated to the sun quiver, define $T(n, m)$ to be the set of all tuples $I=\left(I_{1}, \ldots, I_{m}\right)$ such that $\beta_{I} \neq \beta$ (equivalently, $\left|I_{i}\right|<n$ for some $\left.i\right)$, and $\beta_{I} \circ\left(\beta-\beta_{I}\right)=1$.

We give a description of the set $T(n, m)$ without reference to the sun quiver and only in terms of partitions in Lemma 3.18.

Proposition 3.17. Suppose $\lambda(1), \ldots, \lambda(m)$ are weakly decreasing sequences of $n$ real numbers. For the sun quiver $Q$ and dimension vector $\beta$, the following are equivalent:

1. $\sigma_{1} \in C(Q, \beta)$;

2. $\sum_{i \text { even }}|\lambda(i)|=\sum_{i \text { odd }}|\lambda(i)|$ and

$$
\sum_{j \in I_{i}} \sum_{i} \lambda(i)_{j} \leqslant \sum_{j \in I_{i}} \sum_{i} \lambda(i)_{j}
$$


for every tuple $\left(I_{1}, \ldots, I_{m}\right) \in T(n, m)$.

Proof. We prove the statement by using the definition of the set $T(n, m)$, Theorem 2.42, and Lemma 3.13. Calculating directly from the definition of the weight $\sigma_{1}$ in Equation (3.1),

$\sigma_{1}(\beta)=\sum_{i \text { odd }} \sum_{j=1}^{n-1}\left[\left(\lambda(i)_{j+1}-\lambda(i)_{j}\right) \beta(j, i)\right]+\sum_{i \text { even }} \sum_{j=1}^{n-1}\left[\left(\lambda(i)_{j}-\lambda(i)_{j+1}\right) \beta(j, i)\right]+\sum_{i=1}^{m}(-1)^{i} \lambda(i)_{n} \beta(n, i)$.

Substituting $\beta(j, i)=j, \sigma_{1}(\beta)=0$ precisely when the equality holds. Replacing $\beta$ with $\beta_{I}$, we get a similar expression, and after noting that $\lambda(i)_{j}$ contributes to the inequality exactly when $\beta_{I}(j, i) \neq \beta_{I}(j-1, i)$, meaning $j \in I_{i}$, we obtain the inequality. Because the $\beta_{I}$ are precisely those described in Lemma 3.13, this proves the equivalence.

We now want to better understand the set $T(n, m)$. Specifically, we would like to describe the set without any intrinsic reference to $\beta_{I}$ but rather in terms of partitions. For any tuple $\left(I_{1}, \ldots, I_{m}\right)$ of subsets of $\{1, \ldots, n\}$, define the following decreasing sequences of integers, where we identify $I_{0}$ and $I_{m}$ :

$$
\underline{\lambda}_{1}\left(I_{i}\right)= \begin{cases}\lambda^{\prime}\left(I_{i}\right) & i \text { even } \\ \lambda^{\prime}\left(I_{i}\right)-\left(\left(\left|I_{i}\right|-\left|I_{i-1}\right|-\left|I_{i+1}\right|\right)^{n-\left|I_{i}\right|}\right) & i \text { odd } .\end{cases}
$$

Lemma 3.18. The set $T(n, m)$ for the sun quiver consists of all tuples $I=\left(I_{1}, \ldots, I_{m}\right)$ such that:

(a) at least one of the subsets $I_{1}, \ldots, I_{m}$ has cardinality $<n$;

(b) $\underline{\lambda}_{1}\left(I_{i}\right)$ is a partition for all $1 \leqslant i \leqslant m$;

(c) $f_{1}\left(\underline{\lambda}_{1}\left(I_{1}\right), \ldots, \underline{\lambda}_{1}\left(I_{m}\right)\right)=1$.

Proof. Denote the weight $\left\langle\beta_{1}, \cdot\right\rangle$ by $\sigma_{I}$. Describing $\beta_{1}$ by $\beta_{I}$ as previously and letting $e(j, i)$ be the dimension vector of the simple representation with support at vertex $j$ 
on the $i^{\text {th }}$ flag, the contribution to $\sigma_{I}(l, i)$ at a vertex $l \in\{1, \ldots, n-1\}$ on a flag $i$ is

$$
\left\langle\beta_{I}, e(l, i)\right\rangle=\beta_{I}(l)-\beta_{I}(l-1)
$$

if $i$ is even with $\beta_{I}(0)=0$, and

$$
\left\langle\beta_{I}, e(l, i)\right\rangle=\beta_{I}(l)-\beta_{I}(l+1)
$$

if $i$ is odd. Since $\beta_{I}$ is weakly increasing with jumps of at most one along the flags, this translates to

$$
\sigma_{I}(l, i)=\left\{\begin{array}{rr}
1 & \text { if } l \in I_{i} \\
0 & \text { otherwise }
\end{array}\right.
$$

for $l \in\{1, \ldots, n-1\}$ and $i$ even, and

$$
\sigma_{I}(l, i)=\left\{\begin{array}{lr}
-1 & \text { if } l+1 \in I_{i} \\
0 & \text { otherwise }
\end{array}\right.
$$

for $l \in\{1, \ldots, n-1\}$ and $i$ odd. We then only need to describe the weight at the central vertices. We have

$$
\sigma_{I}(n, i)= \begin{cases}\beta_{I}(n, i)-\beta_{I}(n-1, i) & i \text { even } \\ \beta_{I}(n, i)-\beta_{I}(n, i-1)-\beta_{I}(n, i+1) & i \text { odd },\end{cases}
$$

where $\beta_{I}(n, 0)=\beta_{I}(n, m)$. We use the fact that $\beta_{I}(n, i)=\left|I_{i}\right|$ to finish defining the weight $\sigma_{I}$ in terms of the subsets $I_{i}$, where we identify $I_{0}$ as $I_{m}$ below:

$$
\sigma_{I}(n, i)=\left\{\begin{array}{lr}
0 & i \text { even, } n \notin I_{i}, \\
1 & i \text { even, } n \in I_{i}, \\
\left|I_{i}\right|-\left|I_{i-1}\right|-\left|I_{i+1}\right| & i \text { odd. }
\end{array}\right.
$$

Using this explicit description of this weight $\sigma_{I}$, we can calculate the corresponding partitions in determining $\operatorname{dim} \operatorname{SI}(Q, \beta)_{\sigma_{I}}$. The calculation is done precisely the same as before (see Lemma 3.1 for the details). The partitions we want to consider for describing the contribution of the first $n-1$ vertices along each flag are

$$
\gamma_{n-1}(i)=\left(\beta_{2}(n-1, i)^{(-1)^{i} \sigma_{I}(n-1, i)}, \ldots, \beta_{2}(1, i)^{(-1)^{i} \sigma_{I}(1, i)}\right)^{\prime}, \quad 1 \leqslant i \leqslant m .
$$


Recalling the contributions of the central vertices to the space of semi-invariants, we have that

$$
\gamma(i)=\gamma_{n-1}(i)+\left(\left((-1)^{i} \sigma_{I}(n, i)\right)^{\beta_{2}(n, i)}\right),
$$

are the partitions we want for $1 \leqslant i \leqslant m$, and

$$
\operatorname{dim} \operatorname{SI}\left(Q, \beta_{2}\right)_{\sigma_{I}}=f_{1}(\gamma(1), \ldots, \gamma(m)) .
$$

Since $\beta_{2}=\beta-\beta_{1}$, if $I_{i}=\left\{z_{1}(i)<\ldots<z_{r}(i)\right\}$, then

$$
\beta_{2}\left(z_{j}(i), i\right)=z_{j}(i)-j=z_{j}(i)-1-(j-1)=\beta_{2}\left(z_{j}(i)-1, i\right),
$$

and in particular, $\beta_{2}(n, i)=n-\beta_{1}(n, i)=n-\left|I_{i}\right|$. With this, $\underline{\lambda}_{1}\left(I_{i}\right)=\gamma(i)$ for each $i$.

Thus, if $I=\left(I_{1}, \ldots, I_{m}\right) \in T(n, m)$, then condition (a) is satisfied by definition, (b) is satisfied since $\gamma(i)$ is a partition for each $i$ and $\gamma(i)=\underline{\lambda}_{1}\left(I_{i}\right)$, and (c) is true because

$$
1=\beta_{I} \circ\left(\beta-\beta_{I}\right)=f_{1}(\gamma(1), \ldots, \gamma(m))=f_{1}\left(\underline{\lambda}_{1}\left(I_{1}\right), \ldots, \underline{\lambda}_{1}\left(I_{m}\right)\right) .
$$

Conversely, if conditions (a)-(c) are satisfied by a tuple $I=\left(I_{1}, \ldots, I_{m}\right)$ of subsets of $\{1, \ldots, n\}$, then we can construct a dimension vector $\beta_{I}$ in the usual way and the associated decreasing sequences of integers $\underline{\lambda}_{1}\left(I_{i}\right), 1 \leqslant i \leqslant m$. Necessarily, $\beta_{I} \neq \beta$ and

$$
\beta_{I} \circ\left(\beta-\beta_{I}\right)=f_{1}\left(\underline{\lambda}_{1}\left(I_{1}\right), \ldots, \underline{\lambda}_{1}\left(I_{m}\right)\right)=1
$$

Thus, $I \in T(n, m)$.

Proposition 3.19. Let $\lambda(1), \ldots, \lambda(m)$ be weakly decreasing sequences of $n$ reals, $m \geqslant 4$ and even. The following are equivalent for the sun quiver $Q$ :

1. $\sigma \in C(Q, \beta)$; 
2. the numbers $\lambda(i)_{j}$ satisfy

$$
\sum_{i \text { even }}|\lambda(i)|=\sum_{i \text { odd }}|\lambda(i)|
$$

and

$$
\sum_{j \in I_{i}} \sum_{\text {ieven }} \lambda(i)_{j} \leqslant \sum_{j \in I_{i}} \sum_{\text {odd }} \lambda(i)_{j}
$$

for every tuple $\left(I_{1}, \ldots, I_{m}\right)$ for which $\left|I_{i}\right|<n$ for some $i$, the $\underline{\lambda}_{1}\left(I_{i}\right)$ are partitions, $1 \leqslant i \leqslant m$, and

$$
f_{1}\left(\underline{\lambda}_{1}\left(I_{1}\right), \ldots, \underline{\lambda}_{1}\left(I_{m}\right)\right) \neq 0 ;
$$

3. the numbers $\lambda(i)_{j}$ satisfy the same conditions as in part (2), and it suffices to check only the tuples $\left(I_{1}, \ldots, I_{m}\right)$ which satisfy those conditions and

$$
f_{1}\left(\underline{\lambda}_{1}\left(I_{1}\right), \ldots, \underline{\lambda}_{1}\left(I_{m}\right)\right)=1 .
$$

Proof. This follows from Proposition 3.17, Lemma 3.18, and Remark 2.36.

We can deduce minor conditions on the sizes of the $I_{i}$.

Lemma 3.20. Let $I=\left(I_{1}, \ldots, I_{m}\right)$ be a tuple of subsets of $\{1, \ldots, n\}$ and define $s_{i}$ to be the smallest $k \in\left\{0, \ldots,\left|I_{i}\right|\right\}$ such that $n-k \notin I_{i}$. Then

$$
\max \left\{\left|I_{i-1}\right|,\left|I_{i+1}\right|\right\} \leqslant\left|I_{i}\right| \leqslant\left|I_{i-1}\right|+\left|I_{i+1}\right|+s_{i}
$$

if $I \in T(n, m)$ and $i$ is odd.

Proof. If $\beta_{1} \circ \beta_{2} \neq 0$, then any representation $V$ of dimension $\beta_{1}+\beta_{2}$ has a subrepresentation of dimension $\beta_{1}$. Choosing $V$ such that $V(a)$ is invertible for every arrow $a$ between central vertices, we immediately have

$$
\max \left\{\left|I_{i-1}\right|,\left|I_{i+1}\right|\right\} \leqslant\left|I_{i}\right|
$$


for the stated $i$. Let $\left\langle\beta_{1}, \cdot\right\rangle=\sigma_{1}$. In order for $\operatorname{dim} \operatorname{SI}\left(Q, \beta_{2}\right)_{\sigma_{1}}$ to be nonzero, each $\underline{\lambda}_{1}\left(I_{i}\right)$ must be a partition, meaning, in particular, that it has non-negative parts. Note that $s_{i}$ is precisely the smallest part of $\lambda^{\prime}\left(I_{i}\right)$ for each $i$. We have $\underline{\lambda}_{1}\left(I_{i}\right)=$ $\lambda^{\prime}\left(I_{i}\right)-\left(\sigma_{1}(i)^{n-\left|I_{i}\right|}\right)$ for each of the specified $i$, as well as $\sigma_{1}(i)=\left|I_{i}\right|-\left|I_{i-1}\right|-\left|I_{i+1}\right|$. Thus, $\underline{\lambda}_{1}\left(I_{i}\right)$ is a partition if and only if

$$
0 \leqslant\left|I_{i-1}\right|+\left|I_{i+1}\right|-\left|I_{i}\right|+s_{i}
$$

We note that the proof above does not extend to determining how the sizes of the other subsets compare or whether some subset contains $n$ or is nonempty. This is because no central vertex in the sun quiver shares two flags, so we can't cancel the sizes of any two consecutive subsets that appear in $\sigma_{1}$.

Example 3.21. For the case $n=2$ and $m=6$ for the sun quiver, we can compute all decompositions $\beta=\beta_{1}+\beta_{2}$ such that both $\beta_{1}, \beta_{2}$ are Schur roots and $\beta_{1} \circ \beta_{2}=1$, or equivalently by Theorem 2.42, a description of all facets of $C(Q, \beta)$ in this case. Up to rotations and reflections of the flags, there are 10 total pairs of dimension vectors $\left(\beta_{1}, \beta_{2}\right)$ which provide a decomposition of $\beta$ and such that $\beta_{1} \circ \beta_{2}=1$. Of these pairs, only nine will be Schur. We list the corresponding weights $\sigma_{1}$ in the Appendix, so that the dimension vectors can be calculated easily from them.

The corresponding inequalities of the partitions arising from these weights then provide a complete and minimal list of linear homogeneous inequalities defining when a weight $\sigma$ is in $C(Q, \beta)$ for the sun quiver $Q$ in this case. Specifically, $\sigma \in C(Q, \beta)$ if 
and only if the defining partitions satisfy

$$
|\lambda(1)|+|\lambda(3)|+|\lambda(5)|=|\lambda(2)|+|\lambda(4)|+|\lambda(6)|
$$

and

$$
\begin{aligned}
& \lambda(4)_{1}+\lambda(6)_{1} \leqslant \lambda(1)_{2}+\lambda(3)_{1}+|\lambda(5)| \quad \lambda(2)_{2}+\lambda(4)_{1} \leqslant \lambda(1)_{2}+|\lambda(3)|+\lambda(5)_{2} \\
& \lambda(2)_{2}+\lambda(4)_{1} \leqslant \lambda(1)_{2}+\lambda(3)_{1}+\lambda(5)_{1} \quad \lambda(2)_{2}+\lambda(4)_{1} \leqslant \lambda(1)_{1}+\lambda(3)_{2}+\lambda(5)_{1} \\
& \lambda(4)_{2}+\lambda(6)_{2} \leqslant \lambda(1)_{2}+\lambda(3)_{2}+\lambda(5)_{1} \quad \lambda(4)_{2}+\lambda(6)_{2} \leqslant \lambda(1)_{1}+\lambda(3)_{2}+\lambda(5)_{2}, \\
& \lambda(2)_{1} \leqslant \lambda(3)_{2}+\lambda(1)_{1} \quad \lambda(6)_{2} \leqslant \lambda(1)_{2}+\lambda(5)_{2} \quad|\lambda(2)| \leqslant|\lambda(3)|+\lambda(1)_{1}
\end{aligned}
$$

along with the inequalities obtained by permutations of the flags that respect the symmetries of the sun quiver. This likewise provides a description of all the $\left(I_{1}, \ldots, I_{6}\right)$ in $T(2,6)$, as described in Proposition 3.17.

Example 3.22. The Schur decompositions in the previous example can be used to find certain decompositions for $n>2, m=6$ by using a common shrinking method (see, for instance, [DW00a]). For instance, if $n=3$, we can construct a weight like the second one listed above by extending the dimension vector $\beta_{1}$ by defining the new dimension at the second vertex along each flag to be such that $\sigma_{1}(2, i)=0$. In particular, we get
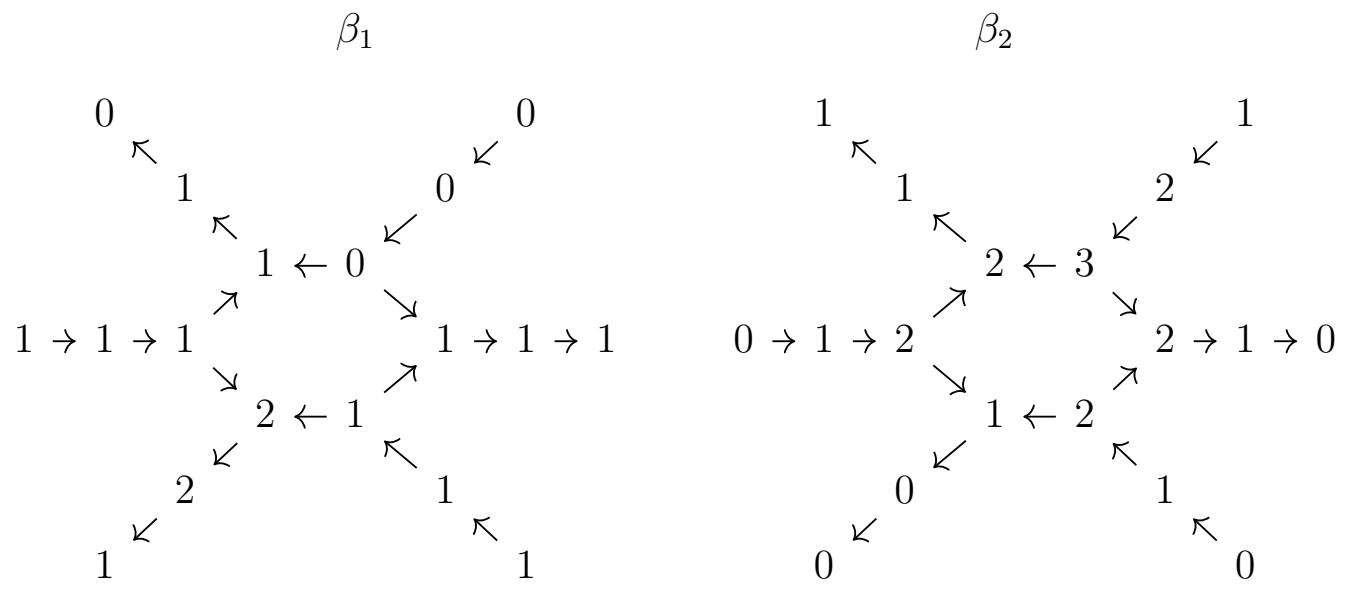


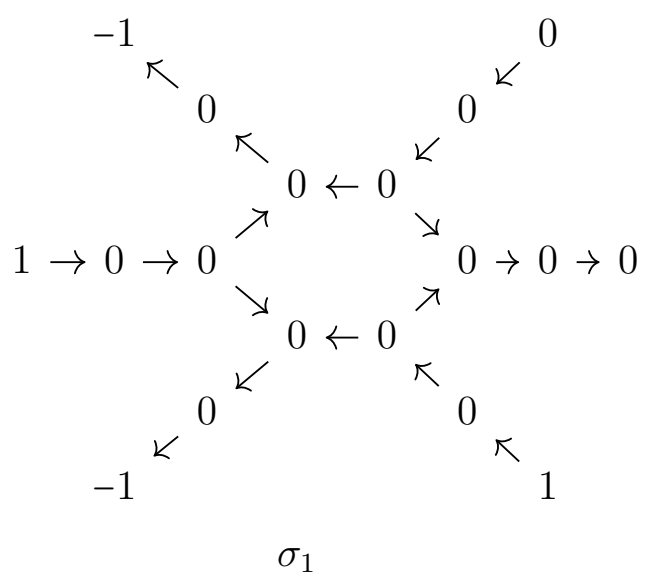

We may shrink $\beta_{1}$ and $\beta_{2}$ along the flags by choosing a representation with the identity as the map between two vertices along a flag with the same dimension; denote the corresponding dimension vectors of the shrunken quiver by $\beta_{1}^{\prime}$ and $\beta_{2}^{\prime}$. It is then easily checked that $\beta_{1}^{\prime}$ is a positive root of $E_{6}$ and $\beta_{2}^{\prime}$ satisfies Kac's inequality, so they are both Schur, and thus so are $\beta_{1}$ and $\beta_{2}$. It is also clear that $\beta_{1} \circ \beta_{2}=1$ in this case. Of course, for each $n>2$ there will be many more Schur decompositions not arising in this way.

\subsubsection{Generalized star quiver}

We repeat the same investigations as in the previous subsection to find the Horn-type inequalities for the nonvanishing of multiplicity (1.3). We will often abbreviate the proofs since many of the calculations are similar. One technicality is that, as we saw in Section 3.1.2, the conditions on an effective weight differ slightly depending on the parity of $m$. This will likewise be reflected in the Horn-type inequalities.

In this section let $\beta_{1}$ be a dimension vector which is weakly increasing with jumps of at most one along each of the flags towards the central vertices for either of the 
generalized star quivers. As before, define the following jump sets:

$$
I_{i}=\left\{l \mid \beta_{1}(l, i)>\beta(l-1, i), 1 \leqslant l \leqslant n\right\}
$$

with the convention $\beta_{1}(0, i)=0$ for all $i$. Each $\beta_{1}$ defines a tuple $I=\left(I_{1}, \ldots, I_{m}\right)$ and conversely, so we denote the defining dimension vector corresponding to the $m$-tuple $I$ as $\beta_{I}$. Note that, however, for the generalized star quiver, we have to also require $\left|I_{1}\right|=\left|I_{2}\right|$ and $\left|I_{m-1}\right|=\left|I_{m}\right|$ since $\beta_{I}(n, 1)=\beta_{I}(n, 2)$ and $\beta_{I}(n, m-1)=\beta_{I}(n, m)$.

Definition 3.23. For the dimension vector $\beta$ associated to the generalized star quiver, define $S(n, m)$ to be the set of all tuples $I=\left(I_{1}, \ldots, I_{m}\right)$ satisfying $\beta_{I} \neq \beta$ (equivalently, $\left|I_{i}\right|<n$ for some $\left.i\right), \beta_{I} \circ\left(\beta-\beta_{I}\right)=1,\left|I_{1}\right|=\left|I_{2}\right|$, and $\left|I_{m-1}\right|=\left|I_{m}\right|$.

Recall the definitions of the weights $\sigma_{2}$ in Equation 3.3 and $\sigma_{2^{\prime}}$ in Equation 3.4 .

Proposition 3.24. Let $Q$ be the generalized star quiver with $m \geqslant 4$ flags and $\beta$ the corresponding dimension vector. Suppose $\lambda(1), \ldots, \lambda(m)$ are weakly decreasing sequences of $n$ real numbers.

1. If $m=2 k$. Then $\sigma_{2} \in C(Q, \beta)$ if and only if

$$
\sum_{i=1}^{k-1}|\lambda(2 i+1)|+|\lambda(2 k)|=|\lambda(1)|+\sum_{i=1}^{k-1}|\lambda(2 i)|
$$

and

$$
\sum_{j \in I_{i}} \sum_{i=1}^{k-1} \lambda(2 i+1)_{j}+\sum_{j \in I_{2 k}} \lambda(2 k)_{j} \leqslant \sum_{j \in I_{1}} \lambda(1)_{j}+\sum_{j \in I_{i}} \sum_{i=1}^{k-1} \lambda(2 i)_{j}
$$

for every tuple $\left(I_{1}, \ldots, I_{2 k}\right) \in S(n, 2 k)$.

2. If $m=2 k+1$, then $\sigma_{2} \in C(Q, \beta)$ if and only if

$$
\sum_{i=1}^{k-2}|\lambda(2 i+1)|=|\lambda(1)|+\sum_{i=1}^{k}|\lambda(2 i)|+|\lambda(2 k+1)|
$$


and

$$
\sum_{j \in I_{i}} \sum_{i=1}^{k-2} \lambda(2 i+1)_{j} \leqslant \sum_{j \in I_{1}} \lambda(1)_{j}+\sum_{j \in I_{i}} \sum_{i=1}^{k} \lambda(2 i)_{j}+\sum_{j \in I_{2 k+1}} \lambda(2 k+1)_{j}
$$

for every tuple $\left(I_{1}, \ldots, I_{2 k+1}\right) \in S(n, 2 k+1)$.

Proof. This is proved similarly as Proposition 3.17. If $m=2 k$, by the definition of $\sigma_{2}$ in Equation (3.3),

$$
\begin{aligned}
\sigma_{2}(\beta)= & \sum_{j=1}^{n-1}\left[\left(\lambda(1)_{j+1}-\lambda(1)_{j}\right) \beta(j, 1)\right]+\sum_{i=1}^{k} \sum_{j=1}^{n-1}\left[\left(\lambda(2 i-1)_{j}-\lambda(2 i-1)_{j+1}\right) \beta(j, 2 i-1)\right] \\
& \left.+\sum_{i=1}^{k-1} \sum_{j=1}^{n-1}\left[\lambda(2 i)_{j+1}-\lambda(2 i)_{j}\right) \beta(j, 2 i)\right]+\sum_{j=1}^{n-1}\left[\left(\lambda(2 k)_{j}-\lambda(2 k)_{j+1}\right) \beta(j, 2 k)\right] \\
& +\left(-\lambda(1)_{n}-\lambda(2)_{n}\right) \beta(n, 1)+\sum_{i=3}^{2 k-2}(-1)^{i+1} \lambda(i)_{n} \beta(n, i)+\left(\lambda(2 k-1)_{n}+\lambda(2 k)_{n}\right) \beta(n, 2 k)
\end{aligned}
$$

with the convention that $\beta(0, i)=0$ for all $i$. Thus, we get the equality in (1), and replacing $\beta$ with $\beta_{I}$ gives the inequality since $\beta(j, i)-\beta(j-1, i)=1$ precisely when $j \in I_{i}$ and 0 otherwise, which proves the equivalence again by Lemma 3.13. The proof for the case $m=2 k+1$ follows similarly with the description of the weight $\sigma_{2^{\prime}}$ in Equation (3.4).

We now want to better understand the set $S(n, m)$. Specifically, we would like to describe this set without any intrinsic reference to $\beta_{I}$ but rather in terms of partitions just as we did for $T(n, m)$ in Lemma 3.18.

In order to construct sequences of weakly decreasing integers $\underline{\lambda}_{2}$ and $\underline{\lambda}_{2}$ for the weights $\sigma_{2}$ and $\sigma_{2^{\prime}}$, respectively, for either an even or odd number of flags for the generalized star quiver, first notice that the exact same equations as (3.5) and (3.6) will hold for $l \in\{1, \ldots, n-1\}$. The central vertices contribute differently to the weight 
depending on the parity of $m$. If $m=2 k$, then

$$
\sigma_{I}(n, i)= \begin{cases}\beta_{I}(n, 2)-\beta_{I}(n, 3) & i=2 \\ \beta_{I}(n, i)-\beta_{I}(n-1, i) & 3 \leqslant i \leqslant 2 k-3, i \text { odd } \\ \beta_{I}(n, i)-\beta_{I}(n, i-1)-\beta_{I}(n, i+1) & 4 \leqslant i \leqslant 2 k-2, i \text { even } \\ \beta_{I}(n, 2 k-1)-\beta_{I}(n-1,2 k-1)-\beta_{I}(n-1,2 k) & i=2 k-1 .\end{cases}
$$

Putting this into the sizes of the subsets, we get

$$
\sigma_{I}(n, i)= \begin{cases}\left|I_{2}\right|-\left|I_{3}\right| & i=2 \\ \left|I_{i}\right|-\left|I_{i} \backslash\{n\}\right| & 3 \leqslant i \leqslant 2 k-3, i \text { odd } \\ \left|I_{i}\right|-\left|I_{i-1}\right|-\left|I_{i+1}\right| & 4 \leqslant i \leqslant 2 k-2, i \text { even } \\ \left|I_{2 k-1}\right|-\left|I_{2 k-1} \backslash\{n\}\right|-\left|I_{2 k} \backslash\{n\}\right| & i=2 k-1 .\end{cases}
$$

The weight for the odd flags other than $i=1$ and $i=2 k-1$ can be simplified to 0 or 1 depending if $n$ is in $I_{i}$ or not, and because $\left|I_{2 k-1}\right|=\left|I_{2 k}\right|$, the weight $\sigma_{I}(n, 2 k-1)$ may be simplified to $\left|I_{2 k-1}\right|-l, l \in\{0,1,2\}$, depending on if $n$ is in $I_{2 k-1}, I_{2 k}$ or both. From this, we define

$$
\underline{\lambda}_{2}\left(I_{i}\right)= \begin{cases}\lambda^{\prime}\left(I_{i}\right) & 1 \leqslant i \leqslant 2 k-3, i \text { odd } \\ \lambda^{\prime}\left(I_{2}\right)-\left(\left(\left|I_{2}\right|-\left|I_{3}\right|\right)^{n-\left|I_{2}\right|}\right) & i=2 \\ \lambda^{\prime}\left(I_{i}\right)-\left(\left(\left|I_{i}\right|-\left|I_{i-1}\right|-\left|I_{i+1}\right|\right)^{n-\left|I_{i}\right|}\right) & 4 \leqslant i \leqslant 2 k-2, i \text { even } \\ \lambda^{\prime}\left(I_{2 k-1}\right)-\left(\left(\left|I_{2 k-1}\right|-\left|I_{2 k-1} \backslash\{n\}-\right| I_{2 k} \backslash\{n\} \mid\right)^{n-\left|I_{2 k-1}\right|}\right) & i=2 k-1 \\ \lambda^{\prime}\left(I_{2 k} \backslash\{n\}\right) & i=2 k .\end{cases}
$$

Similarly, if $m=2 k+1$,

$$
\sigma_{I}^{\prime}(n, i)= \begin{cases}\beta_{I}(n, 2)-\beta_{I}(n, 3) & i=2 \\ \beta_{I}(n, i)-\beta_{I}(n-1, i) & 3 \leqslant i \leqslant 2 k-1, i \text { odd } \\ \beta_{I}(n, i)-\beta_{I}(n, i-1)-\beta_{I}(n, i+1) & 4 \leqslant i \leqslant 2 k-2, i \text { even } \\ \beta_{I}(n, 2 k)-\beta_{I}(n, 2 k-1) & i=2 k,\end{cases}
$$

or in terms of the sizes of the subsets,

$$
\sigma_{I}^{\prime}(n, i)= \begin{cases}\left|I_{2}\right|-\left|I_{3}\right| & i=2 \\ \left|I_{i}\right|-\left|I_{i} \backslash\{n\}\right| & 3 \leqslant 2 k-1, i \text { odd } \\ \left|I_{i}\right|-\left|I_{i-1}\right|-\left|I_{i+1}\right| & 4 \leqslant i \leqslant 2 k-2, i \text { even } \\ \left|I_{2 k}\right|-\left|I_{2 k-1}\right| & i=2 k\end{cases}
$$


Because of this, define

$$
\underline{\lambda}_{2^{\prime}}\left(I_{i}\right)= \begin{cases}\lambda^{\prime}\left(I_{i}\right) & 1 \leqslant i \leqslant 2 k-1, i \text { odd } \\ \lambda^{\prime}\left(I_{2}\right)-\left(\left(\left|I_{2}\right|-\left|I_{3}\right|\right)^{n-\left|I_{2}\right|}\right) & i=2 \\ \lambda^{\prime}\left(I_{i}\right)-\left(\left(\left|I_{i}\right|-\left|I_{i-1}\right|-\left|I_{i+1}\right|\right)^{n-\left|I_{i}\right|}\right) & 4 \leqslant i \leqslant 2 k-2, i \text { even } \\ \lambda^{\prime}\left(I_{2 k}\right)-\left(\left(\left|I_{2 k}\right|-\left|I_{2 k-1}\right|\right)^{n-\left|I_{2 k}\right|}\right) & i=2 k \\ \lambda^{\prime}\left(I_{2 k+1}\right) & i=2 k+1 .\end{cases}
$$

Lemma 3.25. The set $S(n, m)$ for the generalized star quiver consists of all tuples $I=\left(I_{1}, \ldots, I_{m}\right)$ such that

(a) $\left|I_{1}\right|=\left|I_{2}\right|$;

(b) $\left|I_{m-1}\right|=\left|I_{m}\right|$;

(c) at least one of the subsets $I_{1}, \ldots, I_{m}$ has cardinality $<n$;

(d) $\underline{\lambda}_{2}\left(I_{i}\right)$ is a partition for all $1 \leqslant i \leqslant m$ in the case $m=2 k$, and $\underline{\lambda}_{2^{\prime}}\left(I_{i}\right)$ is a partition for all $1 \leqslant i \leqslant m$ in the case $m=2 k+1$;

(e) $f_{2}\left(\underline{\lambda}_{2}\left(I_{1}\right), \ldots, \underline{\lambda}_{2}\left(I_{m}\right)\right)=1$ if $m=2 k$ and $f_{2}\left(\underline{\lambda}_{2^{\prime}}\left(I_{1}\right), \ldots, \underline{\lambda}_{2^{\prime}}\left(I_{m}\right)\right)=1$ if $m=$ $2 k+1$

Proof. The proof is essentially the same as that of Lemma 3.18, so we only need to define the appropriate $\gamma(i)$ and verify $\gamma(i)=\underline{\lambda}_{2}\left(I_{i}\right)$ for all $1 \leqslant i \leqslant m$ if $m=2 k$ and $\gamma(i)=\underline{\lambda}_{2^{\prime}}\left(I_{i}\right)$ if $m=2 k+1$. By the same reasoning as in Lemma 3.5, if $m=2 k$,

$$
\gamma_{n-1}(i)= \begin{cases}\left(\beta_{2}(n-1, i)^{-\sigma_{I}(n-1, i)}, \ldots, \beta_{2}(1, i)^{-\sigma_{I}(1, i)}\right)^{\prime} & i \in\{1,2,4, \ldots, 2 k-2\} \\ \left(\beta_{2}(n-1, i)^{\sigma_{I}(n-1, i)}, \ldots, \beta_{2}(1, i)^{\sigma_{I}(1, i)}\right)^{\prime} & i \in\{3,5, \ldots, 2 k-1,2 k\}\end{cases}
$$

Taking into account the contributions from the central vertices and comparing with the respective dimensions of the spaces of semi-invariants in Equation (3.2) in the proof of Lemma 3.5, the tuples

$$
\gamma(i)= \begin{cases}\left(\beta_{2}(n-1, i)^{-\sigma_{I}(n-1, i)}, \ldots, \beta_{2}(1, i)^{-\sigma_{I}(1, i)}\right)^{\prime} & i=1 \\ \left(\beta_{2}(n-1, i)^{\sigma_{I}(n-1, i)}, \ldots, \beta_{2}(1, i)^{\sigma_{I}(1, i)}\right)^{\prime}+\left((-1)^{i+1} \sigma_{I}(n, i)^{\beta_{2}(n, i)}\right) & 2 \leqslant i \leqslant 2 k-1 \\ \left(\beta_{2}(n-1, i)^{\sigma_{I}(n-1, i)}, \ldots, \beta_{2}(1, i)^{\sigma_{I}(1, i)}\right)^{\prime} & i=2 k\end{cases}
$$


are partitions and $\gamma_{n}(i)=\underline{\lambda}_{2}\left(I_{i}\right)$ for $1 \leqslant i \leqslant m$. The rest of the proof is then similar to that of Lemma 3.18, and the proof for $m=2 k+1$ is completely analogous.

As before, we can deduce minor conditions on the sizes of the $I_{i}$.

Lemma 3.26. Let $I=\left(I_{1}, \ldots, I_{m}\right)$ be a tuple of subsets of $\{1, \ldots, n\}$ and define $s_{i}$ to be the smallest $k \in\left\{0, \ldots,\left|I_{i}\right|\right\}$ such that $n-k \notin I_{i}$. Then

$$
\max \left\{\left|I_{i-1}\right|,\left|I_{i+1}\right|\right\} \leqslant\left|I_{i}\right| \leqslant\left|I_{i-1}\right|+\left|I_{i+1}\right|+s_{i}
$$

if $I \in S(n, m)$ and $i=4,6, \ldots, m-2$ if $m$ is even or $i=4,6, \ldots, m-3$ if $m$ is odd.

Proof. As in Lemma 3.20, choose a representation $V$ of dimension $\beta_{1}+\beta_{2}$ such that $V(a)$ is invertible for every arrow $a$ between central vertices. Then

$$
\max \left\{\left|I_{i-1}\right|,\left|I_{i+1}\right|\right\} \leqslant\left|I_{i}\right|
$$

for the stated $i$. For $j \in\left\{2,2^{\prime}\right\}$, denoting $\left\langle\beta_{1}, \cdot\right\rangle=\sigma_{j}$, in order for $\operatorname{dim} \operatorname{SI}\left(Q, \beta_{2}\right)_{\sigma_{j}}$ to be nonzero, each $\underline{\lambda}_{j}\left(I_{i}\right)$ must be a partition, meaning, in particular, that it has nonnegative parts. Each $s_{i}$ is precisely the smallest part of $\lambda^{\prime}\left(I_{i}\right)$, so we have $\underline{\lambda}_{j}\left(I_{i}\right)=$ $\lambda^{\prime}\left(I_{i}\right)-\left(\sigma_{j}(i)^{n-\left|I_{i}\right|}\right)$ for each of the specified $i$, as well as $\sigma_{j}(i)=\left|I_{i}\right|-\left|I_{i-1}\right|-\left|I_{i+1}\right|$. Thus, $\underline{\lambda}_{j}\left(I_{i}\right)$ is a partition if and only if

$$
0 \leqslant\left|I_{i-1}\right|+\left|I_{i+1}\right|-\left|I_{i}\right|+s_{i} .
$$

We note that this method does not extend to saying anything about the sizes of the other subsets.

\subsection{Generalized eigenvalue problems}

The original motivation in [DW00a] for describing Littlewood-Richardson coefficients in terms of quivers was to provide a solution to a famous conjecture of Horn [Hor62]. 
As we've previously proven corresponding statements for parts (2) and (3) of Theorem 1.2 for the generalized Littlewood-Richardson coefficients $f_{1}$ and $f_{2}$, we now want to find the Weyl-type eigenvalue problem for the non-vanishing of these multiplicities.

\subsubsection{Generalized eigenvalue problem for $f_{1}$}

Recall the construction of the $2 k$-sun quiver in Section 3. The weight for this quiver is

$$
\sigma_{1}(j, i)= \begin{cases}(-1)^{i}\left(\lambda(i)_{j}-\lambda(i)_{j+1}\right) & 1 \leqslant i \leqslant 2 k, 1 \leqslant j \leqslant n-1 \\ (-1)^{i} \lambda(i)_{n} & 1 \leqslant i \leqslant 2 k, j=n .\end{cases}
$$

From Proposition 2.44, $\sigma_{1} \in C(Q, \beta)$ if and only if there is a representation $W \in$ $\operatorname{Rep}(\mathrm{Q}, \beta)$ satisfying the specified matrix equations. These equations are essentially the same as those in Lemma $2.45^{1}$ for a flag $\mathscr{F}(i)$ going out of a central vertex, meaning when $i$ is odd, or in the dual statement when $\mathscr{F}(i)$ is going into a central vertex, meaning when $i$ is even. In either case, the first $n-1$ vertices provide $n \times n$ Hermitian matrices $H^{\prime}(i)$ with eigenvalues

$$
\left(\lambda(i)_{1}-\lambda(i)_{n}, \ldots, \lambda(i)_{n-1}-\lambda(i)_{n}, 0\right)
$$

for each $1 \leqslant i \leqslant m$.

We now consider the equations arising from the central vertices. Denote the $(n-$ $1)^{\text {th }}$ arrow along the $i^{\text {th }}$ flag as simply $b^{i}$ and denote the arrows between the central vertices by the usual partition labeling. The equations arising from the central vertices are, for $1 \leqslant i \leqslant k$,

\footnotetext{
${ }^{1}$ Though the result as stated only applies to flags that are going out from a central vertex. For the flags going into a central vertex, we need to use the dual form of the above lemma. Namely, $\sigma(i)$ is a non-negative number for each $1 \leqslant i \leqslant n-1$, so replace each $-\sigma(i)$ above with $\sigma(i)$, and switch the order of multiplication of $W_{i}$ and $W_{i}^{*}$ in each case for $W_{i} \in \operatorname{Mat}_{(i+1) \times i}(\mathbb{C})$.
} 


$$
\begin{gathered}
W\left(b^{2 i-1}\right)^{*} W\left(b^{2 i-1}\right)-W(\alpha(2 i-1)) W(\alpha(2 i-1))^{*}-W(\alpha(2 i-2)) W(\alpha(2 i-2))^{*}=-\lambda(2 i-1)_{n} \operatorname{Id}_{n}, \\
W(\alpha(2 i-1))^{*} W(\alpha(2 i-1))+W(\alpha(2 i))^{*} W(\alpha(2 i))-W\left(b^{2 i}\right) W\left(b^{2 i}\right)^{*}=\lambda(2 i)_{n} \operatorname{Id}_{n},
\end{gathered}
$$

where $\alpha(0)=\alpha(m)$. We may rewrite these equations by making a few simple observations. Lemma 2.45 gives the Hermitian matrices as $H^{\prime}(i)=W\left(b^{i}{ }^{\star} W\left(b^{i}\right)\right.$, or $W\left(b^{i}\right) W\left(b^{i}\right)^{*}$ depending on the direction of the flag. Clearly, since each $H(i)$ is Hermitian with spectrum

$$
\left(\lambda(i)_{1}-\lambda(i)_{n}, \ldots, \lambda(i)_{n-1}-\lambda(i)_{n}, 0\right)
$$

$H^{\prime}(i)+\lambda(i)_{n} \operatorname{Id}_{n}$ is Hermitian with spectrum $\lambda(i)$; denote this new Hermitian matrix by $H(i)$. We may conjugate the equations by unitary matrices, if necessary. Moreover, for any $n \times n$ matrix $A$, both $A A^{*}$ and $A^{*} A$ are positive semi-definite and have the same spectra, and any positive semi-definite Hermitian matrix $B$ can be written as $W W^{\star}$ or $W^{*} W$, so we may simplify the forms of the equations. We conclude that $\sigma_{1} \in C(Q, \beta)$ if and only if there are Hermitian matrices $H(i)$ with spectra $\lambda(i), 1 \leqslant i \leqslant m$, and positive semi-definite $n \times n$ matrices $B(\alpha(i))$ such that

$$
H(i)=B(\alpha(i))+B(\alpha(i-1)), \quad 1 \leqslant i \leqslant m,
$$

where $B(\alpha(0))=B(\alpha(m))$. Solving for any of the $B(\alpha(i))$ gives $\sum_{i \text { even }} H(i)=$ $\sum_{i \text { odd }} H(i)$. Furthermore, because each $H(i)$ is a sum of positive semi-definite matrices, each $H(i)$ must have non-negative eigenvalues. In addition, we get several other conditions on the Hermitian matrices, namely, we can express alternating sums of an odd number of consecutive indexed matrices as a sum of positive semi-definite matrices. Specifically,

$$
H(i)-H(i+1)+\cdots-H(i+j-1)+H(i+j)=B(i-1)+B(i+j), \quad j \in\{0,2,4, \ldots, 2 k-2\},
$$


where we are taking $H(m+1)=H(1)$, and so on in cyclic fashion. Thus, each such alternating sum is positive semi-definite. (There is, of course, some redundancy in this statement and the previously stated conditions on the $H(i)$.) These are all the conditions on the $H(i)$ which we can conclude from (3.8). Thus, we've found the necessary conditions, stated above, posing the following problem and proving the subsequent statement.

Generalized eigenvalue problem for $f_{1}$. For which weakly decreasing sequences $\lambda(1), \ldots, \lambda(2 k), k \geqslant 2$, of $n$ non-negative real numbers do there exist $n \times n$ complex Hermitian matrices $H(1), \ldots, H(2 k)$ with eigenvalues $\lambda(1), \ldots, \lambda(2 k)$ such that

$$
\sum_{i \text { even }} H(i)=\sum_{i \text { odd }} H(i)
$$

and such that

$$
H(i)-H(i+1)+\cdots-H(i+j-1)+H(i+j), \quad j \in\{0,2, \ldots, 2 k-2\},
$$

has non-negative eigenvalues, where for any $j, H(2 k+j)=H(j)$ ?

Proposition 3.27. Suppose $\lambda(1), \ldots, \lambda(2 k), k \geqslant 2$, are weakly decreasing sequences of $n$ non-negative real numbers, and let $Q$ be the sun quiver, $\beta$ the standard dimension vector, and $\sigma_{1}$ the weight defined in equation (3.1). If $\sigma_{1} \in C(Q, \beta)$, then there exist $n \times n$ complex Hermitian matrices $H(1), \ldots, H(2 k)$ with eigenvalues $\lambda(1), \ldots, \lambda(2 k)$ that solve the generalized eigenvalue problem for the multiplicity $f_{1}$.

While an effective weight defines Hermitian matrices satisfying these conditions, the conditions on the matrices are not sufficient; counterexamples are easily found. For instance, in the case $k=3$, consider

$$
H(1)=\left(\begin{array}{cc}
1 & i \\
-i & 2
\end{array}\right) \quad H(2)=\left(\begin{array}{cc}
2 & 1-i \\
1+i & 1
\end{array}\right) \quad H(3)=\left(\begin{array}{cc}
3 & -i \\
i & 2
\end{array}\right)
$$




$$
H(4)=\left(\begin{array}{cc}
2 & -i \\
i & 3
\end{array}\right) \quad H(5)=\left(\begin{array}{cc}
4 & 1+i \\
1-i & 3
\end{array}\right) \quad H(6)=\left(\begin{array}{cc}
4 & 3 i \\
-3 i & 3
\end{array}\right)
$$

One can check that any decomposition of $H(1)$ into a sum of positive semi-definite matrices $B(1)$ nd $B(2)$ will lead to a matrix $B(i)$ appearing in the decomposition of the other Hermitian matrices which is not positive semi-definite by checking the spectral radius.

Alone, they do not determine a weight because we cannot recapture the decompositions of each $H(i)$ into a sum of the particular positive semi-definite matrices, no canonical choice being available. Any additional conditions would need to record the "linkage" between the consecutive $H(i)$, that is, the fact that they share a common positive semi-definite matrix in their decompositions.

Define the set $\mathcal{K}_{1}(n, m) \subseteq \mathbb{R}^{m n}, m \geqslant 4$ and even, to be all $m$-tuples $(\lambda(1), \ldots, \lambda(m))$ of weakly decreasing sequences of $n$ reals that satisfy $\sum_{i \text { even }}|\lambda(i)|=\sum_{i \text { odd }}|\lambda(i)|$ and

$$
\sum_{j \in I_{i}} \sum_{i \text { even }} \lambda(i)_{j} \leqslant \sum_{j \in I_{i}} \sum_{i \text { odd }} \lambda(i)_{j}
$$

for every tuple $\left(I_{1}, \ldots, I_{m}\right)$ such that the $\underline{\lambda}_{1}\left(I_{i}\right), 1 \leqslant i \leqslant m$, are partitions and

$$
f_{1}\left(\underline{\lambda}_{1}\left(I_{1}\right), \ldots, \underline{\lambda}_{1}\left(I_{m}\right)\right) \neq 0 \text {. }
$$

This makes $\mathcal{K}_{1}(n, m)$ a rational convex polyhedral cone in $\mathbb{R}^{m n}$, which we call the generalized Klyachko's cone for this eigenvalue problem.

Proof of Theorem 1.9. The first and third statements follow from Proposition 3.19, while the second follows from additionally Proposition 3.27. Letting $Q$ denote the sun quiver, there is a map of cones

$$
\mathcal{K}\left({ }_{1} n, m\right) \rightarrow C(Q, \beta) \quad(\lambda(1), \ldots, \lambda(m)) \mapsto \sigma_{1} .
$$


This map is an isomorphism of cones by the chamber inequalities in Lemma 3.13(1) and Proposition 3.27. We found the dimension of $C(Q, \beta)$ to be $m n-1$ in Corollary 3.11, which proves the last statement.

\subsubsection{Generalized eigenvalue problem for $f_{2}$}

Recall the definition of the generalized star quiver in Section 3 and the weights given by equations (3.3) and (3.4), depending on the parity of $m$. By applying the same analysis as in the previous subsection, we reach the same initial conclusions when considering the first $n-1$ vertices of each flag. In this case, keeping the notation as previously, the central vertices produce the following matrix equations for $m=2 k$ :

$$
\begin{gathered}
W\left(b^{1}\right)^{*} W\left(b^{1}\right)+W\left(b^{2}\right)^{*} W\left(b^{2}\right)-W(\alpha(1)) W(\alpha(1))^{*}=\left(-\lambda(1)_{n}-\lambda(2)_{n}\right) \operatorname{Id}_{n} \\
W(\alpha(j-2))^{*} W(\alpha(j-2))+W(\alpha(j-1))^{*} W(\alpha(j-1))-W\left(b^{j}\right) W\left(b^{j}\right)^{*}=\lambda(j)_{n} \operatorname{Id}_{n}, \quad 3 \leqslant j \leqslant m-2, \text { odd } \\
W\left(b^{j}\right)^{*} W\left(b^{j}\right)-W(\alpha(j-1)) W(\alpha(j-1))^{*}-W(\alpha(j-2)) W(\alpha(j-2))^{*}=-\lambda(j)_{n} \operatorname{Id}_{n}, \quad 4 \leqslant j \leqslant m-2, \text { even } \\
W(\alpha(m-3))^{*} W(\alpha(m-3))-W\left(b^{m-1}\right) W\left(b^{m-1}\right)^{*}-W\left(b^{m}\right) W\left(b^{m}\right)^{*}=\left(\lambda(m-1)_{n}+\lambda(m)_{n}\right) \operatorname{Id}_{n}
\end{gathered}
$$

while the matrix equations for $m=2 k+1$ are the same apart from the last equation becoming

$$
W\left(b^{m-1}\right)^{*} W\left(b^{m-1}\right)+W\left(b^{m}\right)^{*} W\left(b^{m}\right)-W(\alpha(m-3)) W(\alpha(m-3))^{*}=\left(-\lambda(m-1)_{n}-\lambda(m)_{n}\right) \operatorname{Id}_{n} .
$$

In either case, using the same reasoning as before, these can be rewritten in the form

$$
\begin{gathered}
H(1)+H(2)=B(\alpha(1)), \quad H(m-1)+H(m)=B(\alpha(m-3)), \\
H(i)=B(\alpha(i-2))+B(\alpha(i-1)) \text { for } 3 \leqslant i \leqslant m-2 .
\end{gathered}
$$

Thus, $H(1)+H(2), H(i), 3 \leqslant i \leqslant m-2$, and $H(m-1)+H(m)$ have non-negative eigenvalues, and

$$
H(1)+\sum_{i=1}^{k-1} H(2 i)=H(m)+\sum_{i=1}^{k-1} H(2 i+1) \quad \text { if } m=2 k
$$




$$
H(1)+H(m)+\sum_{i=1}^{k} H(2 i)=\sum_{i=1}^{k-1} H(2 i+1) \quad \text { if } m=2 k+1 .
$$

Additionally, solving for each of the $B(\alpha(i))$, we get that each of the following is positive semi-definite:

$$
H(1)+H(2), \quad(-1)^{j}(H(1)+H(2))+\sum_{i=3}^{j}(-1)^{j+i} H(i) \quad 3 \leqslant j \leqslant m-2
$$

Of course, other sums will also be positive semi-definite, such as the similarly defined sums that count down from the index $m$ or those of the form $H(i)-H(i+$ $1)+H(i+2)$ for $3 \leqslant i \leqslant m-4$, but these are implied by the above conditions. These conditions complete the list and the generalized eigenvalue problem for the generalized star quiver is then stated and answered in the following.

Generalized eigenvalue problem for $f_{2}$. For which weakly decreasing sequences $\lambda(1), \ldots, \lambda(m), m \geqslant 4$, of $n$ real numbers do there exist $n \times n$ complex Hermitian matrices $H(1), \ldots, H(m)$ with eigenvalues $\lambda(1), \ldots, \lambda(m)$ such that each of the following has non-negative eigenvalues:

$$
\begin{gathered}
H(1)+\sum_{i=1}^{k-1} H(2 i)=H(m)+\sum_{i=1}^{k-1} H(2 i+1), \quad \text { if } m=2 k, \\
H(1)+H(m)+\sum_{i=1}^{k} H(2 i)=\sum_{i=1}^{k-1} H(2 i+1), \quad \text { if } m=2 k+1, \\
H(1)+H(2), \quad(-1)^{j}(H(1)+H(2))+\sum_{i=3}^{j}(-1)^{j+i} H(i) \quad 3 \leqslant j \leqslant m-2
\end{gathered}
$$

for either parity of $m$ ?

Proposition 3.28. Let $\lambda(1), \ldots, \lambda(m), m \geqslant 4$ be weakly decreasing sequences of $n$ real numbers. If $Q$ is the generalized star quiver and $\beta$ is the usual dimension vector, then $\sigma_{2} \in C(Q, \beta)$ if and only if there exist $n \times n$ complex Hermitian matrices 
$H(1), \ldots, H(m)$ with eigenvalues $\lambda(1), \ldots, \lambda(m)$, respectively, that solve the generalized eigenvalue problem for $f_{2}$.

We define the generalized Klyachko's cone for this problem as the rational convex polyhedral cone of $m$-tuples $(\lambda(1), \ldots, \lambda(m))$ of solutions to this generalized eigenvalue problem, and we denote this cone as $\mathcal{K}_{2}(n, m) \subseteq \mathbb{R}^{n m}$.

We can now state the result corresponding to Theorem 1.9 for the generalized star quiver. This is a truer restatement of Horn's conjecture for multiplicity (1.3) as we found necessary and sufficient conditions for the corresponding eigenvalue problem.

Theorem 3.29. Let $\lambda(1), \ldots, \lambda(m), m \geqslant 4$, be weakly decreasing sequences of $n$ real numbers. Then the following are equivalent:

1. $(\lambda(1), \ldots, \lambda(m)) \in \mathcal{K}_{2}(n, m)$;

2. If $m=2 k$, the numbers $\lambda(i)_{j}$ satisfy

$$
\sum_{i=1}^{k-1}|\lambda(2 i+1)|+|\lambda(2 k)|=|\lambda(1)|+\sum_{i=1}^{k-1}|\lambda(2 i)|
$$

and

$$
\sum_{j \in I_{i}} \sum_{i=1}^{k-1} \lambda(2 i+1)_{j}+\sum_{j \in I_{2 k}} \lambda(2 k)_{j} \leqslant \sum_{j \in I_{1}} \lambda(1)_{j}+\sum_{j \in I_{i}} \sum_{i=1}^{k-1} \lambda(2 i)_{j}
$$

for every tuple $\left(I_{1}, \ldots, I_{2 k}\right)$ for which $\left|I_{i}\right|<n$ for some $i,\left|I_{1}\right|=\left|I_{2}\right|$ and $\left|I_{m-1}\right|=$ $\left|I_{m}\right|, \underline{\lambda}_{2}\left(I_{i}\right)$ are partitions, $1 \leqslant i \leqslant m$, and

$$
f_{2}\left(\underline{\lambda}_{2}\left(I_{1}\right), \ldots, \underline{\lambda}_{2}\left(I_{m}\right)\right) \neq 0 \text {. }
$$

If instead $m=2 k+1$, the numbers $\lambda(i)_{j}$ satisfy

$$
\sum_{i=1}^{k-2}|\lambda(2 i+1)|=|\lambda(1)|+\sum_{i=1}^{k}|\lambda(2 i)|+|\lambda(2 k+1)|
$$


and

$$
\sum_{j \in I_{i}} \sum_{i=1}^{k-2} \lambda(2 i+1)_{j} \leqslant \sum_{j \in I_{1}} \lambda(1)_{j}+\sum_{j \in I_{i}} \sum_{i=1}^{k} \lambda(2 i)_{j}+\sum_{j \in I_{2 k+1}} \lambda(2 k+1)_{j}
$$

for every tuple $\left(I_{1}, \ldots, I_{2 k+1}\right)$ for which $\left|I_{i}\right|<n$ for some $i,\left|I_{1}\right|=\left|I_{2}\right|$ and $\left|I_{m-1}\right|=$ $\left|I_{m}\right|, \underline{\lambda}_{2^{\prime}}\left(I_{i}\right)$ are partitions, $1 \leqslant i \leqslant m$, and

$$
f_{2}\left(\underline{\lambda}_{2^{\prime}}\left(I_{1}\right), \ldots, \underline{\lambda}_{2^{\prime}}\left(I_{m}\right)\right) \neq 0
$$

3. the numbers $\lambda(i)_{j}$ satisfy the same conditions as in part (2) where we may restrict to considering only those tuples $\left(I_{1}, \ldots, I_{m}\right)$ which satisfy those conditions and such that

$$
f_{2}\left(\underline{\lambda}_{j}\left(I_{1}\right), \ldots, \underline{\lambda}_{j}\left(I_{m}\right)\right)=1 \quad j \in\left\{2,2^{\prime}\right\}
$$

Moreover, if $\lambda(1), \ldots, \lambda(m)$ are weakly decreasing sequences of $n$ integers, conditions (1) - (3) are equivalent to

4. $f_{2}(\lambda(1), \ldots, \lambda(m)) \neq 0$.

Proof. The equivalence of each part except (1) follows from Proposition 3.24, Lemma 3.25, and Remark 2.36. The implication concerning the generalized Klyachko's cone $\mathcal{K}_{2}(n, m)$ was made in Proposition 3.28.

Proposition 3.30. The generalized Klyachko's cone $\mathcal{K}_{2}(n, m)$ has dimension $m n-1$.

Proof. Letting $Q$ denote either of the generalized star quivers, there is a map of cones

$$
\begin{aligned}
& \mathcal{K}_{2}(n, m) \rightarrow C(Q, \beta) \times \mathbb{R}^{2} \\
& (\lambda(1), \ldots, \lambda(m)) \mapsto\left(\sigma_{j}, \lambda(1)_{n}, \lambda(m)_{n}\right), j \in\left\{2,2^{\prime}\right\} .
\end{aligned}
$$

This map is an isomorphism of cones by the chamber inequalities in Lemma 3.13 and Proposition and 3.28. We showed that $\beta$ is a Schur root in Lemma 3.10, and we found the dimension of $C(Q, \beta)$ to be $m n-3$ in Corollary 3.11, which proves the claim. 


\section{Chapter 4}

\section{The combinatorics and complexity of generalized Littlewood-Richardson coefficients}

In this final chapter, we use the results proved in the previous chapter to further investigate how these generalized coefficients (including (1.4) behave similar to single Littlewood-Richardson coefficients. Specifically, we start by proving a factorization formula for them. We then show that for certain weights they satisfy the same conjectures as single Littlewood-Richardson coefficients that have been so intensely studied, providing evidence that they perhaps always satisfy these conjectures or that they may even be equivalent to single coefficients. Finally, we use Knutson and Tao's LR hives to provide a polytopal description of these multiplicities, which with the saturation properties previously proven, shows that whether they're positive or not can be computed in strongly polynomial time.

\subsection{Factorization formulas}

In this section we adapt Theorem 7.14 in [DW11] to generalized Littlewood-Richardson coefficients in order to provide factorization formulas. To do this for multiplicity (1.4), we recall the necessary results proved in [Chi08]. 
For an $m$-tuple of subsets $I=\left(I_{1}, \ldots, I_{m}\right)$ of $\{1, \ldots, n\}$, define the following weakly decreasing sequences of integers:

$$
\underline{\lambda}_{3}\left(I_{i}\right)= \begin{cases}\lambda^{\prime}\left(I_{i}\right) & i=1 \text { or } i \text { is even } \\ \lambda^{\prime}\left(I_{i}\right)-\left(\left(\left|I_{i}\right|-\left|I_{i+1}\right|-\left|I_{i-1}\right|\right)^{n-\left|I_{i}\right|}\right) & i \leqslant m-2 \text { is odd } \\ \lambda^{\prime}\left(I_{i}\right)-\left(\left(\left|I_{m-1}\right|-\left|I_{m-2}\right|-\left|I_{m} \backslash\{n\}\right|\right)^{n-\left|I_{i}\right|}\right) & i=m-1 \text { is odd } \\ \lambda^{\prime}\left(I_{m}\right) & i=m \text { is odd } \\ \lambda^{\prime}\left(I_{m} \backslash\{n\}\right) & i=m \text { is even. }\end{cases}
$$

Define the set $\mathcal{K}_{3}(n, m) \subseteq \mathbb{R}^{n m}, m \geqslant 3$, to be all $m$-tuples $(\lambda(1), \ldots, \lambda(m))$ of weakly decreasing sequences of $n$ reals that satisfy $\sum_{i \text { even }}|\lambda(i)|=\sum_{i \text { odd }}|\lambda(i)|$ and

$$
\sum_{i \text { even }} \sum_{j \in I_{i}} \lambda(i)_{j} \leqslant \sum_{i \text { odd }} \sum_{j \in I_{i}} \lambda(i)_{j}
$$

for every $m$-tuple $\left(I_{1}, \ldots, I_{m}\right)$ for which $\left|I_{1}\right|=\left|I_{2}\right|,\left|I_{m-1}\right|=\left|I_{m}\right|, \underline{\lambda}_{3}\left(I_{i}\right)$ is a partition for each $1 \leqslant i \leqslant m$, and

$$
f_{3}\left(\underline{\lambda}_{3}\left(I_{1}\right), \ldots, \underline{\lambda}_{3}\left(I_{m}\right)\right) \neq 0 \text {. }
$$

Just like for the sets $\mathcal{K}_{1}(n, m)$ and $\mathcal{K}_{2}(n, m)$, this makes $\mathcal{K}_{3}(n, m)$ a rational convex polyhedral cone. It is shown in Theorem 1.6 [Chi08] that $(\lambda(1), \ldots, \lambda(m)) \in \mathcal{K}_{3}(n, m)$ if and only if this $m$-tuple satisfies the corresponding generalized eigenvalue problem for $f_{3}$, and

$$
(\lambda(1), \ldots, \lambda(m)) \in \mathcal{K}_{3}(n, m) \bigcap \mathbb{Z}^{n m-1} \Longleftrightarrow f_{3}(\lambda(1), \ldots, \lambda(m)) \neq 0
$$

Moreover, by Proposition 1.7 of the same paper, the cone is defined by restricting to the weakly decreasing sequences satisfying the same conditions and simply

$$
f_{3}\left(\underline{\lambda}_{3}\left(I_{1}\right), \ldots, \underline{\lambda}_{3}\left(I_{m}\right)\right)=1
$$

With this notation, we can prove the main theorem of this section. 
Proof of Theorem 1.10. We showed in Theorems 1.9 and 3.29 that an $m$-tuple $(\lambda(1), \ldots, \lambda(m))$ of weakly decreasing sequences of integers being in the respective generalized Klyachko's cones $\mathcal{K}_{1}(n, m)$ and $\mathcal{K}_{2}(n, m)$ is equivalent to

$$
f_{p}(\lambda(1), \ldots, \lambda(m)) \neq 0, \quad p \in\{1,2\} .
$$

As mentioned above, this similarly holds for $f_{3}$. Furthermore, because these multiplicities agree with the dimensions of the respective weight spaces of semi-invariants, we can use the classification of the regular facets (Lemma 3.12 and Lemma 5.2 in [Chi08]) to decompose these multiplicities. Specifically, for any effective weight $\sigma$, the inequality $\sigma\left(\beta_{1}\right) \leqslant 0$ defines a regular facet whenever $\beta_{1} \neq \beta$ is weakly decreasing with jumps of at most one along all the flags and $\beta_{1} \circ\left(\beta-\beta_{1}\right)=1$ (Lemmas 3.13 and 3.15 and Lemma 5.3 in [Chi08]). Each such dimension vector $\beta_{1}$ corresponds to $\beta_{I}$ for some $m$-tuple of subsets $I=\left(I_{1}, \ldots, I_{m}\right)$, and the stated conditions for $\beta_{1}$ translate to the conditions defining the generalized Klyachko's cones by Lemmas 3.18 and 3.25 and Lemma 6.4 in [Chi08], where

$$
(\lambda(1), \ldots, \lambda(m)) \in K_{i}(n, m) \bigcap \mathbb{Z}^{n m-1}, \quad i \in\{1,2,3\},
$$

if and only if this $m$-tuple of weakly decreasing sequences of integers satisfies the respective conditions on each such $m$-tuple $I=\left(I_{1}, \ldots, I_{m}\right)$.

For any of the generalized Littlewood-Richardson coefficients, let $\sigma$ be the weight under consideration (either in Equation (3.1), (3.3), (3.4), or Equations (2)-(5) in [Chi08]). If $\sigma$ is in the interior of the wall, then the $\sigma$-stable decomposition of $\beta$ is $\beta_{1} \dot{+} \beta_{2}$. The weight $\sigma$ is indivisible, so we may use Theorem 2.38 to get, for any $p \in\{1,2,3\}$, $f_{p}(\lambda(1), \ldots, \lambda(m))=\operatorname{SI}(Q, \beta)_{\sigma}=\alpha \circ \beta=\left(\alpha \circ \beta_{1}\right)\left(\alpha \circ \beta_{2}\right)=f_{p}\left(\lambda(1)^{*}, \ldots, \lambda(m)^{*}\right) \cdot f_{p}\left(\lambda(1)^{\#}, \ldots, \lambda(m)^{\#}\right)$. 
If, on the other hand, $\sigma$ is not in the interior of a wall, then the $\sigma$-stable decompositions of $\beta_{1}$ and $\beta_{2}$ are of the form

$$
\beta_{1}=c_{1} \cdot \gamma_{1} \dot{+} \ldots \dot{+} c_{s} \cdot \gamma_{s}, \quad \beta_{2}=d_{1} \cdot \delta_{1} \dot{+} \ldots \dot{+} d_{t} \cdot \delta_{t} .
$$

Thus, the $\sigma$-stable decomposition of $\beta$ is the sum of these. Because the sets $\left\{\gamma_{1}, \ldots, \gamma_{s}\right\}$ and $\left\{\delta_{1}, \ldots, \delta_{t}\right\}$ are disjoint and $\gamma_{i} \circ \delta_{j}=1$ for all $i, j$, for $p \in\{1,2,3\}$, Theorem 2.38 again gives

$$
\begin{aligned}
f_{p}(\lambda(1), \ldots, \lambda(m)) & =\alpha \circ \beta \\
& =\prod\left(\alpha \circ\left(c_{i} \cdot \gamma_{i}\right)\right) \prod\left(\alpha \circ\left(d_{i} \cdot \delta_{i}\right)\right) \\
& =\left(\alpha \circ \beta_{1}\right)\left(\alpha \circ \beta_{2}\right) \\
& =f_{p}\left(\lambda(1)^{*}, \ldots, \lambda(m)^{*}\right) \cdot f_{p}\left(\lambda(1)^{\#}, \ldots, \lambda(m)^{\#}\right) .
\end{aligned}
$$

\subsection{Level-1 weights and stretched polynomials}

In this section we explicitly compute the stretched function for certain weights for the sun and generalized star quivers and verify that similar statements as in the conjectures in Section 1.1.2 for single Littlewood-Richardson coefficients hold for the respective generalized Littlewood-Richardson coefficients. We also note that while similarly defined weights for the star quiver all lie on extremal rays of the cone of effective weights, this is not true for our case.

\subsubsection{Level-1 weights}

In [Fei15], Fei defines a weight for the triple flag star quiver to be level- $m$ if the weight has value $m$ at the central vertex. In Lemma 2.3 of the paper, he classifies all level-1 effective weights and shows that they lie on an extremal ray. For the triple flag star quiver, the Littlewood-Richardson coefficient arising from any effective level-1 weight is of the form $c_{1^{i}, 1^{j}}^{1^{i+j}}$. We use this idea to describe similar weights for the sun quiver. 
We define a level-1 weight to be a (for now, not necessarily effective) nonzero weight with at most one nonzero entry along any flag, with the nonzero entry being 1 for the flags going out and -1 for the flags going in. This will correspond to at most one jump along each flag for the defining dimension vector. Because $\sigma(\beta)=0$ is a necessary condition, if $j_{1}, \ldots, j_{m}$ are the vertices along the flags for which $\sigma\left(j_{i}\right) \neq 0$, counting the vertices towards the central ones (so $j_{i}$ is vertex $\left(j_{i}, i\right)$ ), with $j_{i}=0$ to mean that the weight is trivial along flag $i$, a necessary condition for a weight to be effective for the sun quiver is $\sum_{i \text { odd }} j_{i}=\sum_{i \text { even }} j_{i}$, though this is not sufficient. The condition $\sigma(\beta)=0$ for the generalized star quivers (alternating or not) provide similar necessary but insufficient conditions.

We'll find it useful in this section to describe the effective weights directly in terms of the jumping numbers $j_{i}$ rather than by the conditions found in Proposition 3.27 and 3.28 .

Lemma 4.1. Let $\sigma$ be a level-1 weight for the sun quiver $Q$ and let $j_{1}, \ldots, j_{m}$ be the vertices along flags $1, \ldots, m$ for which $\sigma\left(j_{i}\right) \neq 0$ with $j_{i}=0$ if $\sigma$ is trivial on flag $i$. Then the following are equivalent:

1. $\sigma \in C(Q, \beta)$;

2. $\sum_{i \text { odd }} j_{i}=\sum_{i \text { even }} j_{i}$ and $j_{i}-j_{i+1}+j_{i+2} \geqslant 0$ for $1 \leqslant i \leqslant m$, where $j_{m+1}=j_{1}$ and $j_{m+2}=j_{2}$. Proof. As mentioned above, this immediately follows from Proposition 3.27, however we prove it directly here. Because the partition arising from a flag with the only nonzero weight being 1 or -1 at vertex $j_{i}$ is $\left(1^{j_{i}}\right)$ if $j_{i} \neq 0$ and (0) if $j_{i}=0$, the generalized Littlewood-Richardson coefficient arising from this weight is

$$
f_{1}\left(\left(1^{j_{1}}\right), \ldots,\left(1^{j_{m}}\right)\right)=\sum c_{\alpha_{1}, \alpha_{2}}^{\left(1^{j_{1}}\right)} \cdot c_{\alpha_{2}, \alpha_{3}}^{\left(1^{j_{2}}\right)} \cdots c_{\alpha_{m-1}, \alpha_{m}}^{\left(1^{j_{m}}\right)} \cdot c_{\alpha_{m}, \alpha_{1}}^{\left(1^{j_{m}}\right)}
$$


As observed above, $\sum_{i \text { odd }} j_{i}=\sum_{i \text { even }} j_{i}$ is equivalent to $\sigma(\beta)=0$ and because

$$
\alpha_{i}=j_{i}-\alpha_{i+1}=j_{i}-j_{i+1}+\alpha_{i+2}=j_{i}-j_{i+1}+j_{i+2}-\alpha_{i+3}
$$

the condition $j_{i}-j_{i+1}+j_{i+2} \geqslant 0$ is necessary. It's easy to check that the inequalities on the $j_{i}$ 's imply that $j_{i} \leqslant j_{i+1}$ and $j_{i-1} \leqslant j_{i-2}$ for some $i$, which will be sufficient to prove that such a weight is effective. After reindexing, suppose $j_{1} \leqslant j_{2}$ and $j_{m} \leqslant j_{m-1}$. Then the choice $\alpha(1)=0$ uniquely determines the other $\alpha(i)$ and it follows from the above conditions that each $\alpha(i) \geqslant 0$, making the weight effective.

Similarly, we can derive conditions for the generalized star quiver. Analogously define a level-1 weight for this quiver.

Lemma 4.2. Let $\sigma$ be a level-1 weight for the generalized star quiver and let $j_{1}, \ldots, j_{m}$ be the vertices along the flags for which $\sigma\left(j_{i}\right) \neq 0$, with $j_{i}=0$ if the weight is trivial along flag $i$. Then $\sigma$ lies in $C(Q, \beta)$ if and only if

$j_{1}+\sum_{i=1}^{k-1} j_{i}=\sum_{i=1}^{k-1} j_{2 i+1}+j_{2 k} \quad$ if $m=2 k \quad$ and $\quad j_{1}+j_{2 k+1}+\sum_{i=1}^{k} j_{2 i}=\sum_{i=1}^{k-1} j_{2 i+1} \quad$ if $m=2 k+1$, along with $(-1)^{j}\left(j_{1}+j_{2}\right)+\sum_{i=3}^{j}(-1)^{i+j} j_{i} \geqslant 0,3 \leqslant j \leqslant m-3$, in either case. Furthermore, if these conditions are satisfied, then $\operatorname{dim} \operatorname{SI}(Q, \beta)=1$.

Proof. This follows immediately from Proposition 3.28, or alternatively, may be proven directly. The value of $\operatorname{dim} \operatorname{SI}(Q, \beta)_{\sigma}$ for such a level-1 weight $\sigma$ is

$$
f_{2}\left(\left(1^{j_{1}}\right), \ldots,\left(1^{j_{m}}\right)\right)=\sum c_{\left(1^{j_{1}}\right),\left(1^{j_{2}}\right)}^{\alpha_{1}} \cdot c_{\alpha_{1}, \alpha_{2}}^{\left(1^{j_{3}}\right)} \cdots c_{\alpha_{m-4}, \alpha_{m-3}}^{\left(1^{j_{m-2}}\right)} \cdot c_{\left(1^{j_{m-1}}\right),\left(1^{j_{m}}\right)}^{\alpha_{m-3}} \cdot
$$

The stated equalities and inequalities are then immediately necessary for the weight to be effective. Conversely, these conditions completely determine the $\alpha_{i}$ since each 
partition must be of the form $\left(1^{a_{i}}\right)$ in order for this to be nonzero. In particular, $a_{1}=j_{1}+j_{2}, a_{2}=j_{3}-a_{1}$, and so on, making the $a_{i}$ uniquely determined from the $j_{i}$. Thus, there is only one choice of the $\alpha_{i}$ resulting in a nonzero term of the summation.

Remark 4.3. There are fewer conditions on the $j_{i}$ for the level- 1 weights to be effective than those stated for general weights in the context of the generalized eigenvalue problems for $f_{1}$ in Proposition 3.27. This is because in the general case we can't say that either $H(i)-H(i+1)$ or $H(i+1)-H(i)$ is positive semi-definite, while we can make such a direct comparison of $j_{i}$ and $j_{i+1}$. This allowed us to have an $i$ such that $j_{i} \leqslant j_{i+1}$ and $j_{i-1} \leqslant j_{i-2}$, proving that such a weight was effective. However, this at least tells us that if there is an $i$ such that $H(i+1)-H(i)$ and $H(i-2)-H(i-1)$ are positive semi-definite (along with the other conditions on the $H(i)$ ), then the $H(i)$ solve the generalized eigenvalue problem for $f_{1}$. These conditions, though, are not necessary as examples are easily found.

Remark 4.4. As opposed to the case for the triple flag star quiver, not every effective level-1 weight lies on an extremal ray for the sun quiver. We found several such weights lying on the facets in the case $n=2, m=6$. It can be checked that the first weight in the Appendix provides an instance of a level-1 weight on an extremal ray while the second weight in the first row provides one which is not. If the second weight were on an extremal ray, then Theorem 5.1 in [DW11] says the $\sigma$-stable decomposition would consist of 11 distinct roots, all but at most one being real Schur roots, which is easily calculated to be impossible.

We now want to determine the value $\operatorname{dim} \operatorname{SI}(Q, \beta)_{\sigma}$ for a level-1 weight $\sigma$ for the 
sun quiver.

Lemma 4.5. Let $\sigma \in C(Q, \beta)$ be a level-1 weight for the sun quiver $Q$. Let $j_{1}, \ldots, j_{m}$ be the jumping numbers defining the weight and define $J_{i}=j_{i}-j_{i+1}+j_{i+2}$, where $j_{m+1}=j_{1}$ and $j_{m+2}=j_{2}$. If $s=\min \left\{j_{i}, J_{i} \mid 1 \leqslant i \leqslant m\right\}$, then $\operatorname{dim} \operatorname{SI}(Q, \beta)_{\sigma}=s+1$.

Proof. We first show that $\operatorname{dim} \operatorname{SI}(Q, \beta)_{\sigma} \leqslant s+1$. Clearly, any choice of some $\alpha_{i}=\left(1^{a_{i}}\right)$ completely determines each of the other $\alpha_{j}^{\prime} s$. Moreover, because each partition is of the form $\left(1^{j_{k}}\right), c_{\alpha_{k}, \alpha_{k+1}}^{\left(1^{j_{k}}\right)}=1$ whenever it's nonzero. If $i$ is such that $J_{i}$ or $j_{i}$ is minimal among the set, then consider the factors

$$
c_{\alpha_{i}, \alpha_{i+1}}^{\left(1^{j_{i}}\right)} \cdot c_{\alpha_{i+1}, \alpha_{i+2}}^{\left(1^{j_{i+1}}\right)} \cdot c_{\alpha_{i+2}, \alpha_{i+3}}^{\left(1^{j_{i+2}}\right)}
$$

in the summation. Then $a_{i} \leqslant j_{i}$ and similarly because $a_{i+3}=j_{i+2}-j_{i+1}+j_{i}-a_{i}=J_{i}-a_{i}$, we must have $a_{i} \leqslant J_{i}$ in order for this factor to be nonzero. Hence, there are at most $s+1$ choices for $a_{i}$ resulting in this factor being nonzero.

Suppose $s=j_{i}$ for some $i$. To show equality, we only need to show that $a_{k} \geqslant 0$ for each $k$ for each choice of $a_{i} \in\{0, \ldots, s\}$. This is quickly done since $i$ was chosen to be such that $j_{i} \leqslant j_{k}$ and $j_{i} \leqslant J_{k}$ for each $k$ along with $a_{i} \leqslant j_{i}$. Similarly, if $s=J_{i}$, each $a_{k}$ will be nonnegative after noticing that $j_{i} \leqslant j_{i+1}$ in this case since $J_{i} \leqslant j_{i+2}$. Thus, the only choices for $a_{i}$ resulting in a nonzero term in the summation are $0,1, \ldots, s$, and each such choice results in adding one to the summation.

\subsubsection{Stretched weights}

For a level-1 weight $\sigma$, we'll now investigate the dimensions of the weight spaces of semi-invariants for the stretched weights $N \sigma, N \in \mathbb{Z}^{+}$. If $j_{1}, \ldots, j_{m}$ are the jumping numbers of $\sigma$, the corresponding partitions will be $\left(N^{j_{i}}\right)$. Because $\left|\left(N^{j_{i}}\right)\right|=N j_{i}$ for all 
$i$, Lemma 4.1 generalizes immediately to stretched level-1 weights. Similarly, Lemma 4.5 generalizes in this case because of the Lemma 2.20.

In the proof of the next lemma, we use a partial ordering on the set of rectangular partitions $\left(N^{n}\right)$ for a fixed $N$ defined by $\lambda_{1} \leqslant \lambda_{2}$ to mean that the Young diagram of $\lambda_{1}$ fits inside that of $\lambda_{2}$, meaning $\lambda_{2}-\lambda_{1}$ is a partition. With this, $\lambda_{1} \dot{+} \lambda_{2}$ means stacking the corresponding Young diagrams on top of each other (or in terms of partitions, $\left.\left(N^{n_{1}}\right)+\left(N^{n_{2}}\right)=\left(N^{n_{1}+n_{2}}\right)\right)$, so $\lambda_{3} \leqslant \lambda_{1} \dot{+} \lambda_{2}$ means the Young diagram of $\lambda_{3}$ fits inside the stacked diagrams of $\lambda_{1}$ and $\lambda_{2}$, or equivalently, $\left(N^{n_{1}+n_{2}-n_{3}}\right)$ is a partition. We will use the notation $\lambda_{1} \dot{+}\left(-\lambda_{2}\right)$ to mean we are instead subtracting $\lambda_{2}$ from the bottom of the diagram of $\lambda_{1}$.

Lemma 4.6. For a level-1 weight $\sigma$ for the sun quiver, let $N \in \mathbb{Z}^{+}, j_{1}, \ldots, j_{m}$ the corresponding jumping numbers, and $J_{i}=j_{i}-j_{i+1}+j_{i+2}$ for $i=1, \ldots, m$, with $j_{m+1}=j_{1}$ and $j_{m+2}=j_{2}$. The following are equivalent:

1. $N \sigma \in C(Q, \beta)$;

2. $\sum_{i \text { odd }} j_{i}=\sum_{i \text { even }} j_{i}$ and $J_{i} \geqslant 0$ for all $i$.

If $N \sigma$ is effective, then $\operatorname{dim} \operatorname{SI}(Q, \beta)_{N \sigma}=\left(\begin{array}{c}N+s \\ N\end{array}\right)$, where $s=\min \left\{j_{i}, J_{i} \mid 1 \leqslant i \leqslant m\right\}$.

Proof. As mentioned above, the necessary and sufficient conditions for $N \sigma$ to be effective are proven the same way as in Lemma 4.1. We adapt the proof of Lemma 4.5 to compute the value of the dimension of the weight space for the stretched case.

First suppose $s=j_{i}$, and without loss of generality, suppose $i=1$. The number of partitions $\alpha_{1}$ such that $\alpha_{1} \leqslant\left(N^{j_{1}}\right)$ is $\left(\begin{array}{c}N+j_{1} \\ N\end{array}\right)$. This is because if we consider the Young diagram corresponding to $\left(N^{j_{1}}\right)$, then we choose how many entries of $\alpha_{1}$ have value $N$, then how many have value $N-1$, and so on, which is the same as choosing 
where to place $N$ dividers among $j_{1}$ entries. Because each $\alpha_{1}$ uniquely determines the other $\alpha_{i}$ and because each $c_{\alpha_{i}, \alpha_{i+1}}^{\left(N^{j_{i}}\right)}$ is equal to one when nonzero by Lemma 2.20, $\operatorname{dim} \operatorname{SI}(Q, \beta)_{N \sigma} \leqslant\left(\begin{array}{c}N+j_{1} \\ N\end{array}\right)$. The other direction is proved in a similar way as Lemma 4.5, meaning that each choice of such an $\alpha_{1}$ defines a nonzero product, each of which is necessarily equal to one.

Remark 4.7. The value of $f_{1}(\lambda(1), \ldots, \lambda(m))$ is independent of the value of $n$, the length of each flag. This number can only enlarge the value of the coefficient, which is instead determined by the smallest $j_{i}$ or $J_{i}$. This formula also agrees with the value that we found in Lemma 4.5 since in that case $N=1$.

For a fixed weight $\sigma$ for the sun quiver, we showed in Lemma 3.2 that for each $N \geqslant 1$

$$
f_{1}(N \lambda(1), \ldots, N \lambda(m))=\operatorname{dim} \operatorname{SI}(Q, \beta)_{N \sigma},
$$

where $\lambda(1), \ldots, \lambda(m)$ are the partitions arising from $\sigma$ as stated in equation (3.1). Clearly, this is a polynomial as each stretched function of a single Littlewood-Richardson coefficient is a polynomial (see [DW02]). The above formula allows us to calculate $\operatorname{dim} \operatorname{SI}(Q, \beta)_{N \sigma}$ for any level-1 weight immediately.

Proposition 4.8. Let $\lambda(1), \ldots, \lambda(2 k), k \geqslant 2$, be partitions of at most $n$ parts and of the form $\left(1^{j_{i}}\right)$ if $j_{i} \neq 0$ and zero if $j_{i}=0$ for some integers $0 \leqslant j_{1}, \ldots, j_{m} \leqslant n$. Suppose the $j_{i}$ satisfy the following conditions:

1. $\sum_{i \text { odd }} j_{i}=\sum_{i \text { even }} j_{i}$

2. $J_{i}:=j_{i}-j_{i+1}+j_{i+2} \geqslant 0$, where $j_{2 k+1}=j_{1}, j_{2 k+2}=j_{2}$. 
Then for any $N \in \mathbb{Z}_{>0}$, the stretched Littlewood-Richardson polynomial $f_{1}(N \lambda(1), \ldots, N \lambda(2 k))$ is equal to $\left(\begin{array}{c}N+s \\ N\end{array}\right)$, where $s=\min \left\{j_{i}, J_{i} \mid 1 \leqslant i \leqslant 2 k\right\}$. If either (1) or (2) is not satisfied, then $f_{1}(N \lambda(1), \ldots, N \lambda(2 k))=0$.

Proof. This follows immediately from Lemma 4.6 and Theorem 1.7.

As we will show, the stretched Littlewood-Richardson coefficients for the generalized star quiver are severely restricted when each partition is stretched by the same factor. The proof of Lemma 4.2 extends to stretched weights $N \sigma$ with the same necessary and sufficient conditions for the weight to be effective.

As mentioned in Lemma 4.2, in the case that each partition is of the form $\left(1^{j_{i}}\right)$ and the level-1 weight $\sigma$ is effective, a term of the summation

$$
f_{2}\left(\left(1^{j_{1}}\right), \ldots,\left(1^{j_{m}}\right)\right)=\sum c_{\left(1^{j_{1}}\right),\left(1^{j_{2}}\right)}^{\alpha_{1}} \cdot c_{\alpha_{1}, \alpha_{2}}^{\left(1^{j_{3}}\right)} \cdots c_{\alpha_{m-4}, \alpha_{m-3}}^{\left(1^{j_{m-2}}\right)} \cdot c_{\left(1^{j_{m-1}}\right),\left(1^{j_{m}}\right)}^{\alpha_{j_{m-3}}}
$$

is nonzero only if $\alpha_{1}=\left(1^{j_{1}+j_{2}}\right)$, and because each $\alpha_{i}$ is of the form $\left(1^{a_{i}}\right)$, the other $\alpha_{i}$ are likewise immediately determined by the $j_{i}$.

Proposition 4.9. Let $\lambda(1), \ldots, \lambda(m), m \geqslant 4$, be partitions with at most $n$ parts and of the form $\left(1^{j_{i}}\right)$ if $j_{i} \neq 0$ or zero if $j_{i}=0$ for some integers $0 \leqslant j_{i} \leqslant n$. Suppose the $j_{i}$ satisfy the following conditions:

1. $j_{1}+\sum_{i=1}^{k-1} j_{2 i}=\sum_{i=1}^{k-1} j_{2 i+1}+j_{2 k}$ if $m=2 k$ or $j_{1}+j_{2 k+1}+\sum_{i=1}^{k} j_{2 i}=\sum_{i=1}^{k-1} j_{2 i+1}$ if $m=2 k+1$;

2. $(-1)^{j}\left(j_{1}+j_{2}\right)+\sum_{i=3}^{j}(-1)^{i+j} j_{i} \geqslant 0,3 \leqslant j \leqslant m-3$.

For any $N \in \mathbb{Z}_{>0}$, the stretched Littlewood-Richardson polynomial $f_{2}(N \lambda(1), \ldots, N \lambda(m))=1$. 
Proof. For consistency, we use $\left(1^{j_{i}}\right)$ for the zero partition if $j_{i}=0$. We have

$f_{2}(N \lambda(1), \ldots, N \lambda(m))=f_{2}\left(\left(N^{j_{1}}\right), \ldots,\left(N^{j_{m}}\right)\right)=\sum c_{\left(N^{j_{1}}\right),\left(N^{j_{2}}\right)}^{\alpha_{1}} \cdot c_{\alpha_{1}, \alpha_{2}}^{\left(N^{j_{3}}\right)} \ldots c_{\alpha_{m-4}, \alpha_{m-3}}^{\left(N^{j_{m-3}}\right)} \cdot c_{\left(N^{j_{m-1}}\right),\left(N^{j_{m}}\right)}^{\alpha_{m-1}}$.

By Lemmas 2.20 and 2.21, each nonzero term in the summation is equal to one. Without loss of generality, suppose $j_{1} \geqslant j_{2}$. The first Lemma states that a nonzero term must have $\alpha_{1}$ to be of the form

$$
\alpha_{1}=\left(N+c_{1}, N+c_{2}, \ldots, N+c_{j_{2}}, N, \ldots, N, N-c_{j_{2}}, \ldots, N-c_{1}\right),
$$

with $N \geqslant c_{1} \geqslant \ldots \geqslant c_{j_{2}} \geqslant 0$. However, $\alpha_{1}$ must also fit inside of $\left(N^{j_{3}}\right)$, so $c_{1}=\cdots=c_{j_{2}}=$ 0 . Similarly for $\alpha_{m-3}$. Therefore, $\alpha_{1}$ and $\alpha_{m-3}$ are completely determined by the $j_{i}$, which then completely determine $\alpha_{i}$ for $2 \leqslant i \leqslant m-4$. There is then only one nonzero term in the summation, which has value one by the Lemmas.

Proposition 4.10. Let $\lambda(1), \ldots, \lambda(m), m \geqslant 3$, be partitions with at most $n$ parts and of the form ( $\left.1^{j_{i}}\right)$ if $j_{i} \neq 0$ or zero if $j_{i}=0$ for some integers $0 \leqslant j_{i} \leqslant n$. Suppose the $j_{i}$ satisfy the following conditions:

1. $\sum_{i \text { odd }} j_{i}=\sum_{i \text { even }} j_{i}$

2. $\sum_{k=1}^{i}(-1)^{i+k} j_{k}$ for all $2 \leqslant i \leqslant m-2$ whenever $m>3$.

Then for any $N \in \mathbb{Z}_{>0}$, the stretched Littlewood-Richardson polynomial satisfies $f_{3}(N \lambda(1), \ldots, N \lambda(m))=1$.

Proof. The stated conditions are those for the generalized eigenvalue problem for multiplicity (1.4), as shown in Theorem 1.6 of [Chi08]. By the saturation property (Theorem 1.4, [Chi08]), the stretched weight is likewise nonzero when these conditions hold. It's immediately checked that whenever the stretched multiplicity is nonzero it 
must be equal to one in the case that all the partitions are of the stated form since in this case $\alpha_{1}=\left(N^{j_{2}-j_{1}}\right)$, and likewise the other $\alpha_{i}$ are uniquely determined.

Remark 4.11. Propositions 4.8, 4.9, and 4.10 show that the conjectures in Section 1.1.2 hold for generalized Littlewood-Richardson coefficients of these forms and for level-1 weights. It would be interesting to see if similar conjectures for these generalized coefficients hold for all weights.

\subsection{Polytopal description and complexity}

In this section we examine the complexity of the generalized Littlewood-Richardson coefficients by defining polytopes whose number of lattice points are equal to the respective multiplicities. The main result is Theorem 1.8, which states that the positivity of each of the multiplicities, that is, whether or not they are zero, can be calculated in strongly polynomial time.

The proof of deciding the positivity of a Littlewood-Richardson coefficient relies on two main points: a polyhedral interpretation of these numbers and the saturation theorem. While we define a polytope for the generalized Littlewood-Richardson coefficients to prove a similar result, it would be nice to have a purely combinatorial algorithm, such as those of max-flow or weighted matching problems in combinatorial optimization. Much work has been made towards this for single LittlewoodRichardson coefficients (see [BI09], [BI13], and [Ike16]).

\subsubsection{Polytopal description}

In order to first determine the complexity of the positivity of multiplicity (1.2), we will define a polytope by determining a system of homogeneous linear inequalities 
whose number of integer-valued solutions is precisely this multiplicity. The idea is to use the Littlewood-Richardson hives defined by Knutson and Tao in [KT99] and "glue" the LR hives together appropriately.

To define the polytope associated with multiplicity (1.2), subdivide a regular $m$ gon into $m$ triangles with $n+1$ vertices along each exterior edge and a common vertex at the center. Subdivide each of these triangles into $n^{2}$ triangles of the same size, so the regular $m$-gon is divided into $m n^{2}$ total triangles. For $1 \leqslant r \leqslant m$, we label the edges in the $r^{\text {th }}$ triangular array in the same way as in Section 2.1.2. That is, the first subscript $i$ refers to the row from bottom to top while the second subscript $j$ refers to the diagonal from left to right, and $0 \leqslant i, j \leqslant n-1$. The edges along increasing diagonals are labeled $e_{i j}$, the edges along decreasing diagonals are labeled $f_{i j}$, and the horizontal edges are $g_{i j}$. The superscript $r$ refers to which triangular array is used, though this is often omitted.

Let $E$ be the set of hive edges and $\mathbb{R}^{E}$ the labelings of these edges by real numbers. Define an $(m, n)$-LR sun hive to be a regular $m$-gon subdivided into $m$ triangular arrays with $n+1$ vertices along each edge that satisfies the rhombus inequalities (2.2) and the border conditions

$$
\sum_{i=0}^{n-1} e_{i 0}^{r}+\sum_{i=0}^{n-1} f_{i n-i}^{r}=\sum_{j=0}^{n-1} g_{0 j}
$$

for each $1 \leqslant r \leqslant m$. It is integral if the labeling lies in $\mathbb{Z}^{E}$. These inequalities define a convex polyhedral cone, denoted $C \subseteq \mathbb{R}^{E}$. An $(m, n)$-LR sun hive then consists of $m$ LR hives with $n$ edges along each side of the boundary of a regular $m$-gon and with the respective conditions and appropriately shared sides. Let $B$ be the set of border edges $g_{0 j}^{k}$ for $1 \leqslant r \leqslant m, 0 \leqslant j \leqslant n-1$, and $\rho: \mathbb{R}^{E} \rightarrow \mathbb{R}^{B}$ the restriction map of an LR 
sun hive to its border. For each $b \in \mathbb{R}^{B}$, the fiber $\rho^{-1}(b) \cap C$ is a compact polytope, called the $m$-sun hive polytope over $b$.

Because one LR hive is used to calculate one Littlewood-Richardson coefficient, it stands to reason that "gluing" multiple LR hives together appropriately should be used to calculate our generalized Littlewood-Richardson coefficients. Before stating and proving this we first make precise how we intend to "glue" the LR hives. Given one LR hive we combine it with another LR hive by requiring the two to share a side other than the base. This results in the second LR hive being flipped. Of course, we need to verify the values assigned to the shared edges coincide and make precise the edge labelings along with the rhombus inequalities for the flipped hive. For the flipped hive the edges on a descending diagonal are now labeled by the $e$ 's while the ascending diagonal edges are labeled by the $f^{\prime}$ 's. For instance, if $n=3$, such a gluing of the two LR hives would be

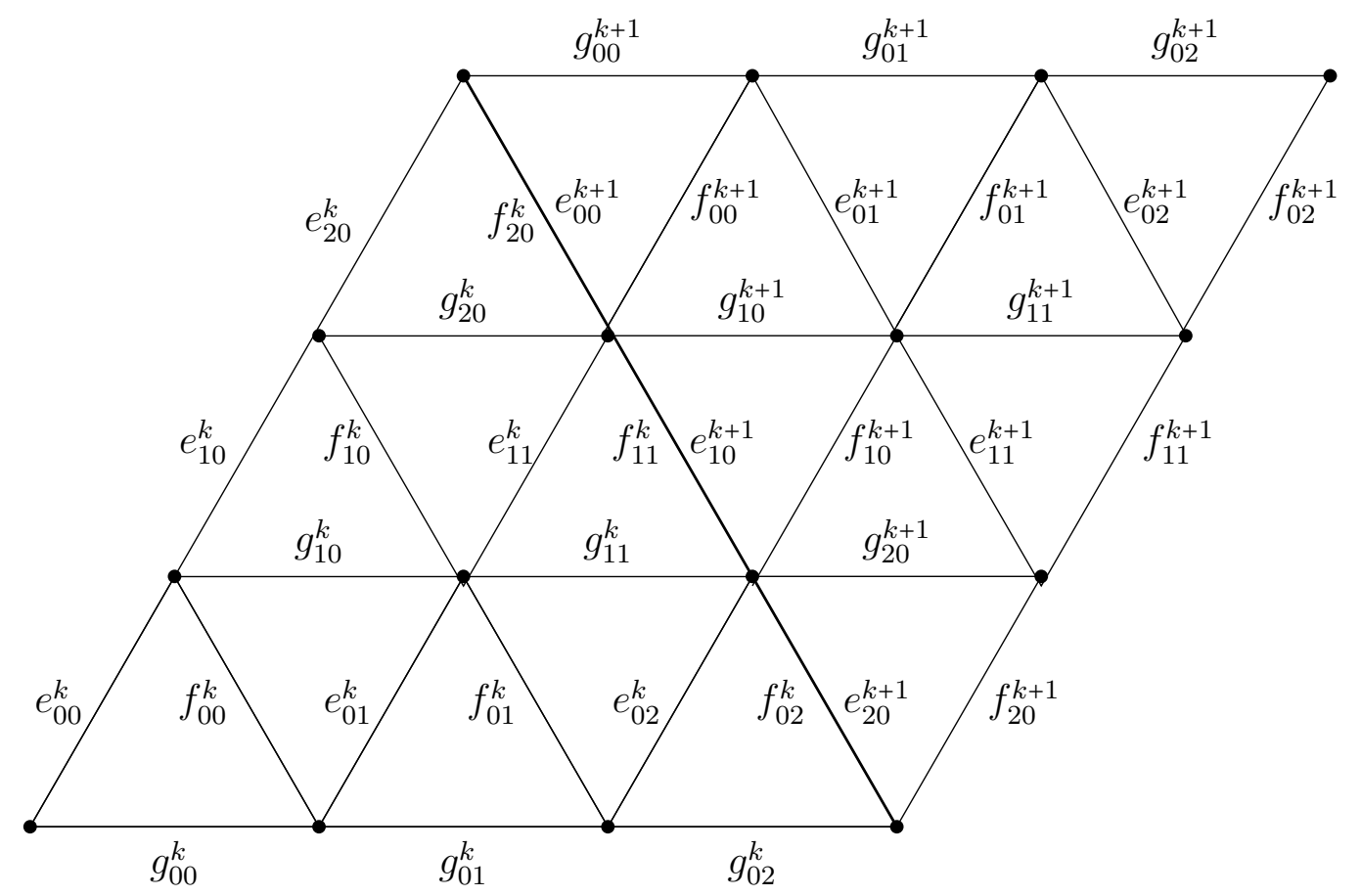


Under this notation, $e_{j 0}^{k+1}=f_{n-1-j j}^{k}$. Flipping the triangular arrays causes each type of rhombus to be flipped, but by also switching the labels for the $e$ 's and $f^{\prime} \mathrm{s}$ the same rhombus inequalities in (2.2) hold. For these flipped arrays we will want to specify when $n$-tuples $\lambda, \mu, \nu$ such that $|\nu|=|\lambda|+|\mu|$ determine the border and align along shared edges, but we will wait to do this depending on which side of the flipped hive we want to have labeled $\nu$.

Remark 4.12. When defining the $(m, n)$-LR sun hive the rhombus inequalities did not include those two types arising from rhombi of adjacent triangles from different hives. Because one hive is flipped, there is no natural way of determining which direction the inequality should be and the direction may differ in different examples. Interestingly, though, the direction of the inequalities arising from adjacent hives is the same within each individual example examined.

With this notation we may now prove the polytopal description of the generalized Littlewood-Richardson coefficient (1.2).

Theorem 4.13. For partitions $\lambda(1), \ldots, \lambda(2 k), k \geqslant 2$, of no more than $n$ parts, the generalized Littlewood-Richardson coefficient

$$
\sum c_{\alpha(1), \alpha(2)}^{\lambda(1)} c_{\alpha(2), \alpha(3)}^{\lambda(2)} \cdots c_{\alpha(2 k-1), \alpha(2 k)}^{\lambda(2 k-1)} c_{\alpha(2 k), \alpha(1)}^{\lambda(2 k)}
$$

is equal to the number of integer $(2 k, n)-L R$ sun hives with external boundary labels determined by the $\lambda(i)$ in cyclic orientation (so that the edge labeled $\lambda(r)$ is between the edges labeled $\lambda(r+1)$ and $\lambda(r-1))$. For instance, the boundary labels of a $(6, n)-L R$ 
sun hive are

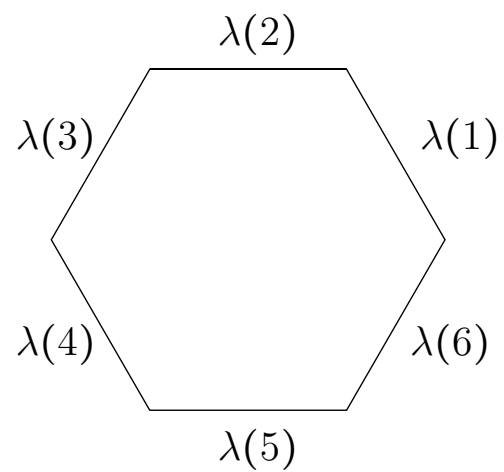

Proof. By Theorem 2.23 the Littlewood-Richardson coefficient $c_{\alpha(r), \alpha(r+1)}^{\lambda(r)}$ is equal to the number of integer LR $n$-hives with $\lambda(r)$ as the base which satisfy the boundary conditions and rhombus inequalities, where $\alpha(r), \alpha(r+1)$ are some tuples of no more than $n$ parts forming the other two sides of the $r^{\text {th }}$ triangular array. Necessarily the tuple $\alpha(r)$ is also a boundary of the $(r-1)^{t h}$ triangular array while $\alpha(r+1)$ is a boundary of the $(r+1)^{t h}$. We use the previously defined notation for each hive and adjacent (flipped) hive, so we only need to specify the border labels. If the base labeled $\lambda(r)$ has edges labeled $\lambda(r)_{1}, \ldots, \lambda(r)_{n}$ from left to right, then the adjacent base labeled $\lambda(r+1)$ has edges labeled $\lambda(r+1)_{1}, \ldots, \lambda(r+1)_{n}$ from right to left. In this way, edges labeled by $\alpha(r)$ and $\alpha(r+1)$ in the $r^{\text {th }}$ LR hive are $\alpha(r)_{1}, \ldots, \alpha(r)_{n}, \alpha(r+1)_{1}, \ldots, \alpha(r+1)_{n}$ clockwise while the edges in the adjacent hive are labeled $\alpha(r+2)_{1}, \ldots, \alpha(r+2)_{n}, \alpha(r+1)_{1}, \ldots, \alpha(r+1)_{n}$ counterclockwise. The multiplicity

$$
\sum c_{\alpha(1), \alpha(2)}^{\lambda(1)} c_{\alpha(2), \alpha(3)}^{\lambda(2)} \cdots c_{\alpha(2 k-1), \alpha(2 k)}^{\lambda(2 k-1)} c_{\alpha(2 k), \alpha(1)}^{\lambda(2 k)}
$$

is then equal to the number of integer $(2 k, n)$-LR sun hives with these choices of $\alpha(1), \ldots, \alpha(2 k)$. The total number of integer $(2 k, n)$-LR sun hives with the boundaries $\lambda(1), \ldots, \lambda(2 k)$ is then the sum over all possible integer tuples $\alpha(1), \ldots, \alpha(2 k)$ 
with at most $n$ parts.

Remark 4.14. There is a characterization of LR hives with vertex labels rather than edge labels, which is the preferred labeling in [KT99]. Though the two labelings may be used interchangeably for all results concerning a single Littlewood-Richardson coefficient, the vertex labeling fails in the case of the generalized coefficients because the vertices along a shared boundary would not necessarily agree. For instance, the vertex at the center of the regular $n$-gon could only be zero while this would force the external boundary labels to not be the $\lambda(i)$.

Remark 4.15. In the previous theorem, it is necessary that the number of partitions be at least four and even. We saw that adjacent LR hives must "flip" in order to line up the boundary edges and in our description the edges on the side determined by $\lambda(i)$ are labeled by $\lambda(i)_{1}, \ldots, \lambda(i)_{n}$ from left to right for odd $i$ and in reverse order for even $i$. If the number of partitions, $m$, were odd, then the first and $m+1$ hives are the same, yet these have different parities, so we get two different labelings. The number of hives must then be even and it is easily checked that $m=2$ fails.

As we've seen, the rhombus inequalities (2.2) and boundary conditions (2.3) determine a polytope whose number of lattice points corresponds to the multiplicity (1.2) when the external boundaries are determined by the $\lambda(r)$. For each $1 \leqslant r \leqslant 2 k$, these inequalities may be solved into a linear program $A_{r} \mathbf{x}_{\mathbf{r}} \leqslant \mathbf{b}_{\mathbf{r}}$, where $A_{r}$ is a matrix with entries $0,1,-1, \mathbf{x}_{\mathbf{r}}$ is the vector of interior edges $e_{i j}^{r}, f_{i j}^{r}, g_{i j}^{r} 0 \leqslant i \leqslant n-1,0 \leqslant j \leqslant n-1$, and the entries of $\mathbf{b}_{\mathbf{r}}$ are homogeneous, linear forms in the entries of $\lambda(r)$ (which are integral when $\lambda(r)$ is a partition). Because this can be done for each $r$, we can express 
all of these as a single linear program $A \mathbf{x} \leqslant \mathbf{b}$, where $A$ is the block sum of the matrices $A_{r}$ and similarly for $\mathbf{x}$ and $\mathbf{b}$. Again, $\mathbf{b}$ will be homogeneous, which is necessary for the proof of the complexity of the positivity of the generalized Littlewood-Richardson coefficient. In this way, the multiplicity is equal to the number of integer-valued vector solutions $\mathbf{x}$ to this inequality. This proves the following.

Lemma 4.16. For partitions $\lambda(1), \ldots, \lambda(2 k), k \geqslant 2$, there exists a linear program $A \mathbf{x} \leqslant \mathbf{b}$, where the matrix $A$ has entries $0,1,-1, \mathbf{b}$ is a vector of homogeneous, integral, linear forms in terms of the parts of $\lambda(1), \ldots, \lambda(2 k)$, and the multiplicity

$$
\sum c_{\alpha(1), \alpha(2)}^{\lambda(1)} c_{\alpha(2), \alpha(3)}^{\lambda(2)} \cdots c_{\alpha(2 k-1), \alpha(2 k)}^{\lambda(2 k-1)} c_{\alpha(2 k), \alpha(1)}^{\lambda(2 k)}
$$

is equal to the number of solution vectors $\mathbf{x}$ with integer entries.

With this, we can prove the complexity of the positivity of this multiplicity.

Proposition 4.17. Determining whether the multiplicity (1.2) is positive or not can be decided in strongly polynomial time in the sense of [Tar86].

Proof. First, we claim that the $m$-sun hive polytope, whose number of lattice points equals

$$
f_{1}(\lambda(1), \ldots, \lambda(2 k))=\sum c_{\alpha(1), \alpha(2)}^{\lambda(1)} c_{\alpha(2), \alpha(3)}^{\lambda(2)} \cdots c_{\alpha(2 k-1), \alpha(2 k)}^{\lambda(2 k-1)} c_{\alpha(2 k), \alpha(1)}^{\lambda(2 k)}
$$

contains an (integer) $(2 k, n)$-LR sun hive if and only if it is nonempty, which is equivalent to the multiplicity being nonzero. Note that because the polytope is defined by a linear system $A \mathbf{x} \leqslant \mathbf{b}$ where $\mathbf{b}$ is homogeneous (Lemma 4.16), for any integer $N$, $f_{1}(N \lambda(1), \ldots, N \lambda(2 k))$ is the number of integer $(2 k, n)$-LR sun hives in the polytope with scaled external boundaries. 
One direction of the claim is trivial, so suppose the polytope is nonempty. In particular, the polytope has a vertex. One characterization of a vertex of a polytope (Lemma 2.24) defined by such a system of inequalities $A \mathbf{x} \leqslant \mathbf{b}$ is a point $\mathbf{v}$ of the polytope such that $A \mathbf{v}=\mathbf{b}$. Because $A$ is of full rank (because the defined polytope is nonempty) and the entries of $A$ and $\mathbf{b}$ are all integers, Cramer's rule implies that all the vertices of the polytope have rational coefficients. There is then an integer $N$ for which the scaled polytope contains a $(2 k, n)$-LR sun hive. The saturation theorem 1.7 ensures that $f_{1}(\lambda(1), \ldots, \lambda(m))$ is positive, so the original polytope contains a $(2 k, n)$-LR sun hive.

Determining whether the polytope is nonempty or not can be determined in polynomial time using linear programming, such as the ellipsoid or interior point algorithm. Furthermore, because the linear program $A \mathbf{x} \leqslant \mathbf{b}$ is combinatorial, positivity can be determined in strongly polynomial time by using the algorithm in [Tar86].

We can apply the same techniques to find a polytopal description of the other two multiplicities and use them to determine similar results about the complexity of their positivity.

Theorem 4.18. Let $\lambda(1), \ldots, \lambda(m)$ be partitions with at most $n$ parts.

1. The generalized Littlewood-Richardson coefficient

$$
\sum c_{\alpha(1), \alpha(2)}^{\lambda(1)} \cdot c_{\alpha(2), \alpha(3)}^{\lambda(2)} \cdots c_{\alpha(2 k-1), \alpha(2 k)}^{\lambda(2 k-1)} \cdot c_{\alpha(2 k), \alpha(1)}^{\lambda(2 k)}
$$

is equal to the number of $(2 k, n)-L R$ sun hives for $k \geqslant 2$. In particular, the multiplicity (1.2) for the first branching rule for the diagonal embedding of GL( $n)$ is equal to the number of $(6, n)-L R$ sun hives of the form 


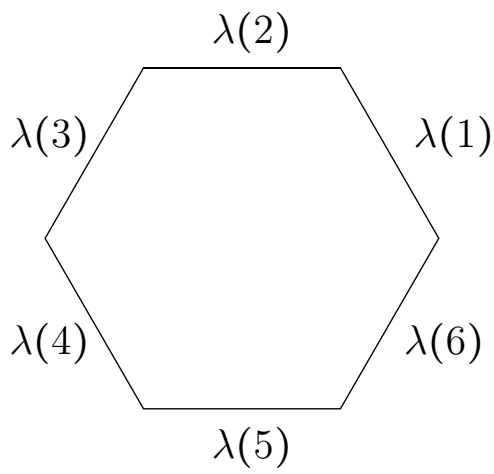

2. The generalized Littlewood-Richardson coefficient

$$
\sum c_{\lambda(1), \alpha(1)}^{\lambda(2)} \cdot c_{\alpha(1), \alpha(2)}^{\lambda(3)} \cdots c_{\alpha(m-4), \alpha(m-3)}^{\lambda(m-2)} \cdot c_{\alpha(m-3), \lambda(m)}^{\lambda(m-1)}
$$

for $m \geqslant 4$ is the number of $(m-2)-L R$ hives of the form
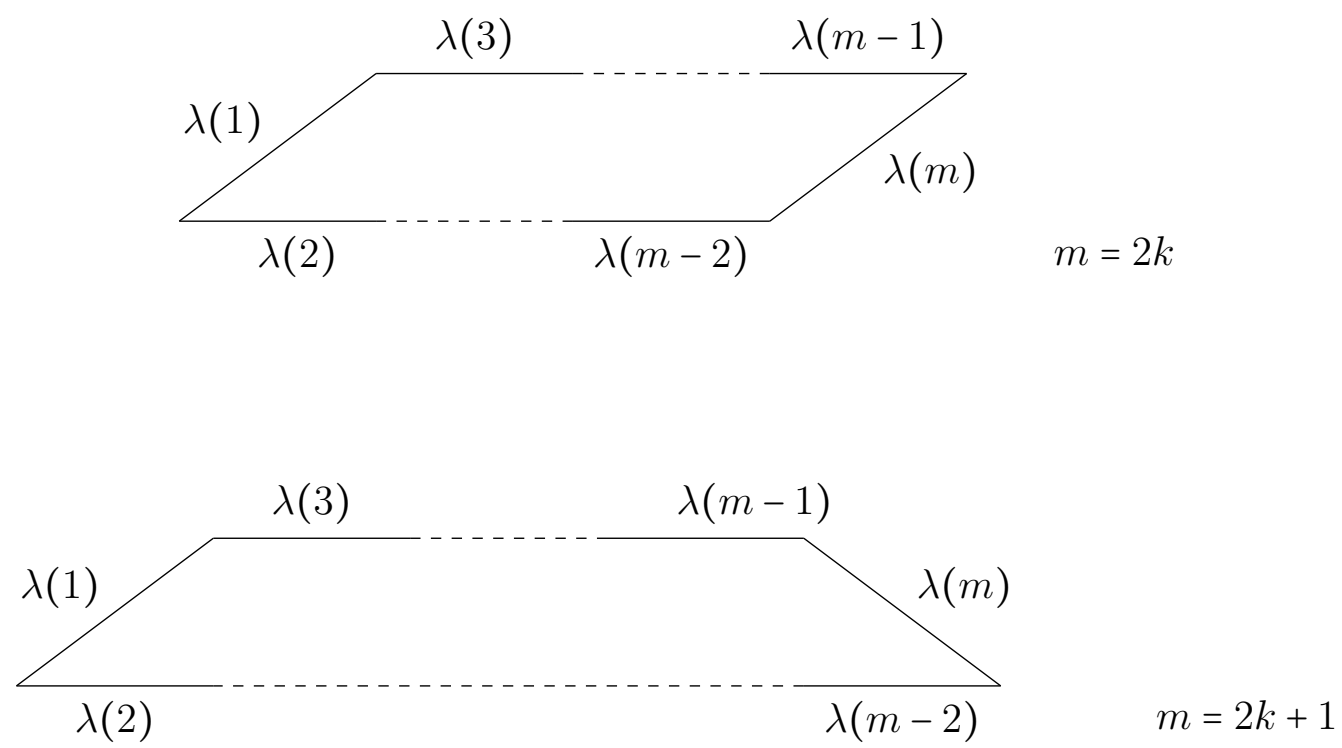

In particular, this provides a polytopal description of the multiplicity (1.4) for extremal weight crystals when $m=6$.

3. The generalized Littlewood-Richardson coefficient

$$
\sum c_{\lambda(1), \lambda(2)}^{\alpha(1)} \cdot c_{\alpha(1), \alpha(2)}^{\lambda(3)} \cdots c_{\alpha(m-4), \alpha(m-3)}^{\lambda(m-2)} \cdot c_{\lambda(m-1), \lambda(m)}^{\alpha(m-3)}
$$


for $m \geqslant 4$ is equal to the number of $(m-2)$-LR hives of the same form as in (2) except with $\overline{\lambda(i)}$ replacing $\lambda(i)$ for $i=1,2, m-1, m$. In particular, the multiplicity (1.3) for the branching rule for the direct sum embedding of $\mathrm{GL}(n)$ (1.3) is equal to the number of 4-LR hives of the form

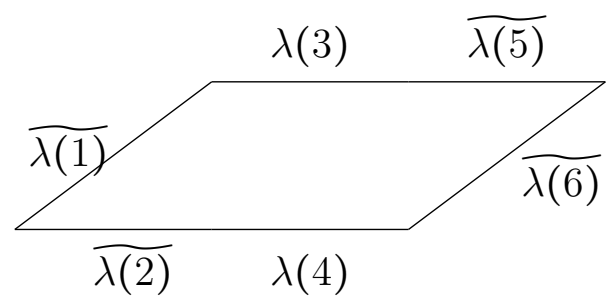

Proof. The first statement is precisely Theorem 4.13. To prove (3) we first use the twisted partitions $\widetilde{\lambda(i)}$ to rewrite each summand as

$$
c_{\overline{\lambda(1)}, \alpha(1)}^{\overline{\lambda(2)}} c_{\alpha(1), \alpha(2)}^{\lambda(3)} \cdots c_{\alpha(m-4), \alpha(m-3)}^{\lambda(m-2)} c_{\alpha(m-3), \lambda(m)}^{\lambda(\overline{\lambda(m-1)}} .
$$

(We leave the labeling for the partitions $\alpha(1)$ and $\alpha(m-3)$ unchanged since we are summing over all possible such partitions with at most $n$ parts and twisting the partition does not affect the number of parts.) With this we can use the edge labels for each LR hive so that adjacent hives align after we "flip" every other hive. The rest of the proof then follows as before and (2) is proven similarly (without the twisted partitions).

Remark 4.19. The polygons in (2) and (3) may be replaced by a regular $m$-gon just as in (1), but with two adjacent parts missing. The border labels are then arranged in cyclic fashion with $\lambda(1), \lambda(2)$ providing the external boundaries next to one missing piece and $\lambda(m-1), \lambda(m)$ the external boundaries next to the other missing piece (or 
the twisted partitions for (3)). For instance,

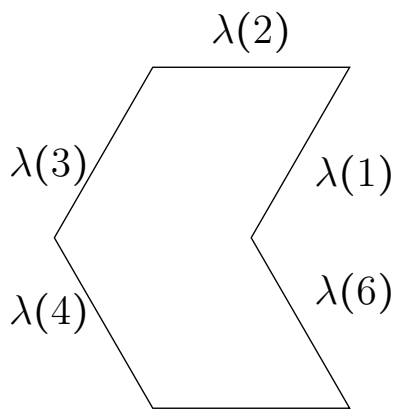

$\lambda(5)$

describes the polygon for the multiplicity in (2) when $m=6$, and the same polygon describes the multiplicity in (3) if we use the twisted partitions as described.

Now that we have a polytopal description of the multiplicities we can prove the complexity of the positivity of each of these multiplicities.

Proof of Theorem 1.8. The proof for the first branching rule multiplicity and its generalization is precisely that of Proposition 4.17. In a similar way, the rhombus inequalities arising from the polytopal descriptions for the other two multiplicities may be solved into linear programs of the appropriate form. By using the saturation properties of these other multiplicities (Theorem 3.7 and Theorem 1.4 of [Chi08]), the rest of the proof follows in the same way. 


\section{Appendix}

The following are the weights $\sigma_{1}$ that correspond to a complete and minimal list of

Schur roots $\beta_{1}$ and $\beta_{2}$ such that $\beta=\beta_{1}+\beta_{2}$ and $\beta_{1} \circ \beta_{2}=1$ in the case $(n, m)=(2,6)$, up to permutations of the flags respecting the symmetries of the sun quiver.
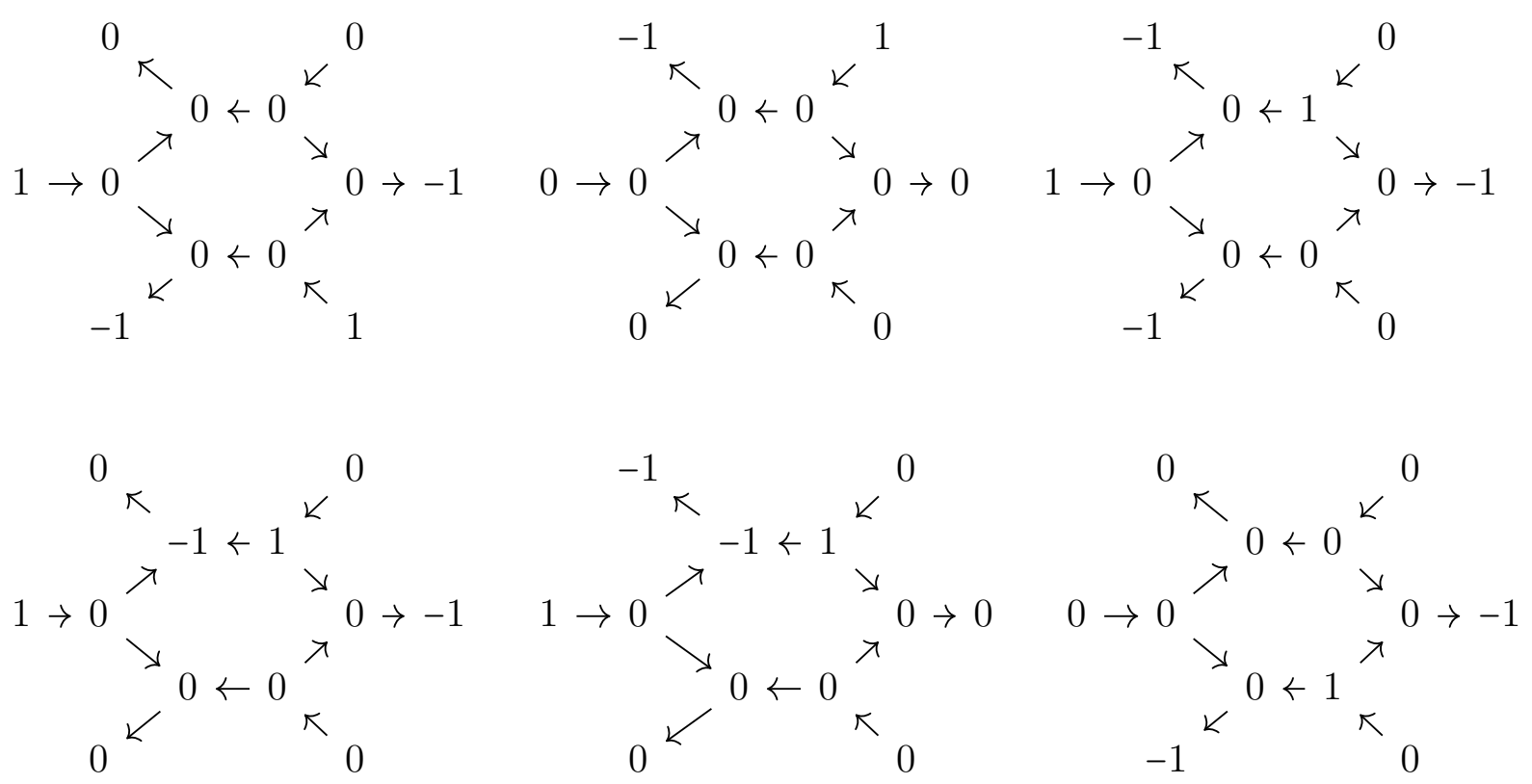

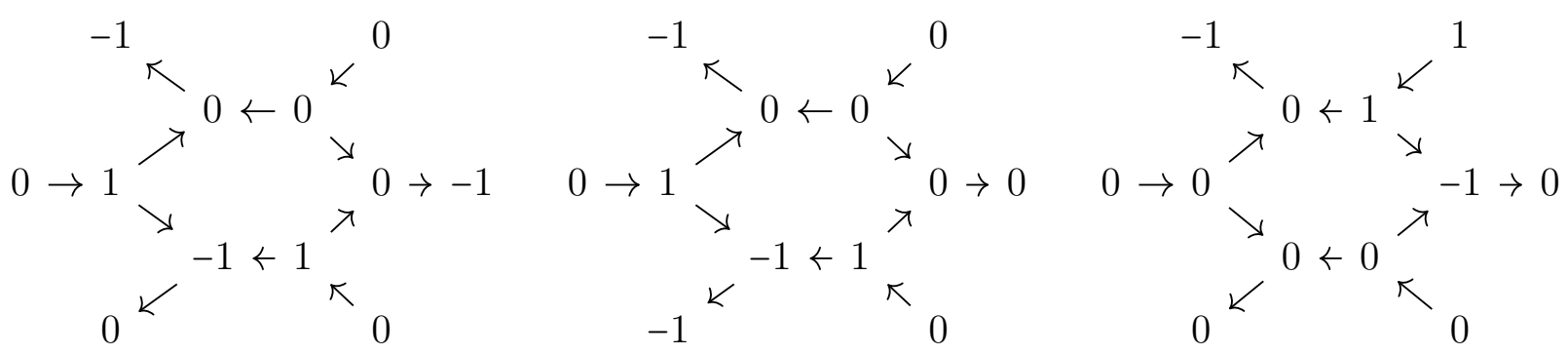




\section{Index of symbols}

\begin{tabular}{|c|c|}
\hline$I_{i}, 69$ & $\underline{\operatorname{dim}} V, 37$ \\
\hline$N \lambda, 3$ & $\operatorname{det}_{V}^{r}, 18$ \\
\hline$Q^{\lambda} V, 21$ & $\mathrm{GL}(\beta), 38$ \\
\hline$S^{\lambda} V, 2,21$ & $\mathcal{K}(n, 3), 5$ \\
\hline$T(n, m), 69$ & $\mathcal{K}_{1}(n, m), 13,85$ \\
\hline$V^{\lambda}, 20$ & $\mathcal{K}_{2}(n, m), 88$ \\
\hline$W_{2}(Q, \beta), 48$ & $\mathcal{K}_{3}(n, m), 91$ \\
\hline$X^{*}(\mathrm{GL}(\beta)), 39$ & $\lambda(I), 2$ \\
\hline $\mathscr{F}(i), 55$ & $\lambda^{\prime}, 19$ \\
\hline $\mathbb{H}(\beta), 46$ & $\langle\alpha, \beta\rangle, 38$ \\
\hline$\Lambda_{n}, 24$ & $\mathcal{L R}(n, 3), 5$ \\
\hline $\operatorname{Rep}(\mathrm{Q}, \beta), 38$ & $\mathcal{D}, 65$ \\
\hline$\Sigma(Q, \beta), 40$ & $\mathcal{F}(Q, \beta), 51$ \\
\hline$\alpha \circ \beta, 45$ & $\nu / \lambda, 28$ \\
\hline$\alpha \hookrightarrow \beta, 45$ & $\mathcal{P}_{r}, 19$ \\
\hline$\alpha_{1} \dot{+} \ldots \dot{+} \alpha_{s}, 47$ & $\sigma$-semi-stable, 43 \\
\hline$\bigwedge^{r} V, 18$ & $\sigma$-stable, 43 \\
\hline$C(Q, \beta), 46$ & $\sigma(\alpha), 43$ \\
\hline
\end{tabular}


$\sigma(x), 40$

$\sigma_{1}, 59$

$\sigma_{2}, 63$

$\sigma_{2^{\prime}}, 63$

$\operatorname{SI}(Q, \beta), 39$

$\mathrm{SI}(Q, \beta)_{\sigma}, 39$

$\operatorname{Rep}(Q), 37$

$\mathrm{Sym}^{r} V, 18$

$\operatorname{rep}(Q), 37$

$\underline{\lambda}_{1}, 70$

$\underline{\lambda}_{1}\left(I_{i}\right), 13$

$\underline{\lambda}_{3}, 91$ $\widetilde{\lambda}, 29$

$c^{V}, 40$

$c_{\lambda(1), \lambda(3)}^{\lambda(2)}, 27$

$c_{\lambda(1), \lambda(3)}^{\lambda(2)}, 2$

$c_{1} \cdot \alpha_{1} \dot{+} \ldots \dot{+} c_{s} \cdot \alpha_{s}, 47$

$c_{W}, 40$

$e_{T}, 22$

$f_{1}(\lambda(1), \ldots, \lambda(m)), 10$

$f_{2}(\lambda(1), \ldots, \lambda(m)), 10$

$f_{3}(\lambda(1), \ldots, \lambda(m)), 10$

$s_{\lambda}, 23$ 


\section{Index}

$\sigma$-semi-stable, 44

$\sigma$-stable, 44

$\sigma$-stable decomposition, 47

algebraic group, 17

bit length, 7

branching rule, 9

chamber inequalities, 67

cone of effective weights, 46

conjugate partition, 19

content, 28

dimension vector, 37

dominant weight, 24

Euler form, 38

exchange, 20

facet, 47

$$
\text { regular, } 65
$$

trivial, 65

generalized eigenvalue problem

$$
\begin{aligned}
& f_{1}, 84 \\
& f_{2}, 87
\end{aligned}
$$

generalized Littlewood-Richardson

$$
\text { coefficients, } 9
$$

Geometric Complexity Theory, 7

hive

$(m, n)$-LR sun hive, 103

$m$-sun hive polytope, 104

integral, 34

LR, 34

model, 33

polytope, 34

Klyachko's cone, 5

generalized, 13, 85

level-1 weight, 94 
Littlewood-Richardson coefficient, 3, 27

Littlewood-Richardson rule, 28

partition, 2, 19

polyhedron, 35

polytope, 35

quiver, 37

generalized star, 61

star, 51

sun, 56

rational characters, 39

regular inequalities, 68

representation

determinant, 18

linear, 18

morphism, 37

polynomial, 18

quiver, 37

rational, 18

simple, 37

trivial, 18

weight, 24 reverse lattice word condition, 28

rhombus inequalities, 33

Schur

function, 23

module, 21

representation, 46

root, 46

semi-invariant, 25, 39

semi-invariants of weight $\sigma, 39$

stretched polynomial, 6

strongly polynomial time, 8

subrepresentation, 37

weight, 39

integral effective, 40

weight space decomposition, 39

Weyl's eigenvalue problem, 1

Young diagram, 19

Young tableau, 20

semistandard, 22

skew, 28

standard, 22 


\section{Bibliography}

[AW98] S. Agnihotri and C. Woodward. Eigenvalues of products of unitary matrices and quantum Schubert calculus. Math. Res. Lett., 5(9): 817-836 (1998).

[Bel01] P. Belkale. Local systems on $\mathbb{P}^{1} \backslash S$ for $S$ a finite set. Compositio Math., 129(1): 67-86 (2001).

[Bel06] P. Belkale. Geometric proofs of Horn and saturation conjectures. J. Algebraic Geom. 15(1): 133-173 (2006).

[Bel07] P. Belkale Geometric proof of a conjecture of Fulton. Advances in Mathematics, 216: 346-357 (2007).

[Buc00] A. Buch. The saturation conjecture (after A. Knutson and T. Tao). Enseign. Math., 46(2), no. 1-2: 46-60 (2000).

[BI09] P. Bürgisser, C. Ikenmeyer. A max-flow algorithm for positivity of LittlewoodRichardson coefficients. DMTCS Proc., AK, 267-278 (2009).

[BI13] P. Bürgisser, C. Ikenmeyer. Deciding positivity of Littlewood-Richardson coefficients. SIAM J. Discrete Math., 27(4): 1639-1681 (2013).

[Chi06] C. Chindris. Eigenvalues of Hermitian matrices and cones arising from quivers. Int. Math. Res. Not. (2006).

[Chi08] C. Chindris. Quivers, long exact sequences, and Horn type inequalities. J. Algebra, 320(1): 128-157 (2008).

[Chi09] C. Chindris. Quivers, long exact sequences, and Horn type inequalities II. Glasg. Math. J., 51(2): 201-217 (2009).

[CG02] W. Crawley-Boevey, Ch. Geiss. Horn's problem and semi-stability for quiver representations. Representations of algebra, I, II: 40-48, 2002.

[DW00a] H. Derksen, J. Weyman. Semi-invariants of quivers and saturation for Littlewood-Richardson coefficients. J. Amer. Math. Soc., 13(3): 467-479 (2000).

[DW00b] H. Derksen, J. Weyman. On the $\sigma$-stable decomposition of quiver representations. Preprint, http://www.math.lsa.umich.edu/ hderksen/preprints/ stab.pdf (2000).

[DW02] H. Derksen, J. Weyman. On Littlewood-Richardson polynomials. J. Algebra, 255(2): 247-257 (2002). 
[DSW07] H. Derksen, A. Schofield, J. Weyman. On the number of subrepresentations of a general quiver representation. J. Lond. Math. Soc., 76(2): 135-147 (2007).

[DW11] H. Derksen, J. Weyman. The combinatorics of quiver representations. Ann. Inst. Fourier (Grenoble), 61(3): 1061-1131 (2011).

[Fei15] J. Fei. Cluster algebras and semi-invariant rings: triple flags. Preprint, https: //arxiv.org/pdf/1411.4693.pdf (2015).

[FH91] W. Fulton and J. Harris. Representation theory. A first course. Graduate Texts in Mathematics, volume 129 (1991).

[Ful97a] W. Fulton. Young tableaux. With applications to representation theory and geometry. London Mathematical Society Student Texts, volume 35 (1997).

[Ful97b] W. Fulton. Eigenvalues of sums of Hermitian matrices. Séminaire N. Bourbaki, 845: 255-269 (1997-98)

[Ful98] W. Fulton. Intersection theory. Springer, volume 2 (1998).

[Ful00] W. Fulton. Eigenvalues of majorized Hermitian matrices and LittlewoodRichardson coefficients. Linear Algebra Appl., 319(1-3): 23-36, 2000.

[GLS93] M. Grotschel, L. Lovasz, A. Schrijver. Geometric algorithms and combinatorial optimization. Algorithms and Combinatorics, 2. Springer-Verlag (1993).

[Hap84] D. Happel. Relative invariants of quivers of tame type. J. Algebra, 86(2): 315-335 (1984).

[HJ09] R. Howe, S. Jackson, et. al. Toric degeneration for branching algebras. Adv. Math., 220(6): 1809-1841 (2009).

[Hor62] A. Horn. Eigenvalues of sums of Hermitian matrices. Pacific J. Math., 12: 225-241 (1962).

[How95] R. Howe. Perspectives on invariant theory: Schur duality, multiplicity-free actions and beyond. The Schur Lectures (1992), Math. Conf. Proc.,8: 1-182 (1995).

[HTW05] R. Howe, E. Tan, J. Willenbring. Stable branching rules for classical symmetric pairs. Trans. Amer. Math. Soc., 357(4): 1601-1626 (2005).

[Ike16] C. Ikenmeyer. Small Littlewood-Richardson coefficients. J. Algebraic Combin., 44(1): 1-29 (2016).

[Kac82] V. Kac. Infinite root systems, representations of graphs and invariant theory II. J. Algebra, 78(1): 141-162 (1982).

[Kas90] M. Kashiwara. Crystalizing the $q$-analogue of universal enveloping algebras. Comm. Math. Phys., 133(2): 249--260 (1990).

[Kin70] R. King. Generalized Young tableaux and the general linear group. J. Math. Phys. 11: 280-293 (1970). 
[Kin71] R. King. Modification rules and products of irreducible representations for the unitary, orthogonal, and symplectic groups. J. Math. Phys. 12: 1588-1598 (1971).

[Kin94] A. D. King. Moduli of representations of finite-dimensional algebras. Quart. J. Math. Oxford Ser. (2), 45(180): 515-530 (1994).

[Kle68] T. Klein. The multiplication of Schur-functions and extension of $p$-modules. J. London Math. Soc., 43: 280-284 (1968).

[Kly98] A. Klyachko. Stable bundles, representation theory and Hermitian operators. Selecta Math. (N.S.), 4(3): 419-445 (1998).

[Koi89] K. Koike. On the decomposition of tensor products of the representations of the classical groups. Adv. Math. 74(1): 57-86 (1989).

[KT99] A. Knutson, T. Tao. The honeycomb model of $\mathrm{gl}_{n}(\mathbb{C})$ tensor products. I. Proof of the saturation conjecture. J. Amer. Math. Soc., 12(4): 1055-1090 (1999).

[KT01] A. Knutson, T. Tao. Honeycombs and sums of Hermitian matrices. Not. Am. Math. Soc. 48(2):175-186, 2001.

[KTT04] R. King, C. Tollu, F. Toumazet. Stretched Littlewood-Richardson and Kostka coefficients. Symmetry in physics, 34: 99-112 (2004).

[KTT06a] R.C. King, C. Tollu, F. Toumazet. The hive model and the factorisation of Kostka coefficients. Sém. Lothar. Combin., 54A (2006).

[KTT06b] R.C. King, C. Tollu, F. Toumazet. The hive model and the polynomial nature of stretched Littlewood-Richardson coefficients. Sém. Lothar. Combin., $54 \mathrm{~A}(2006)$.

[KTW04] A. Knutson, T. Tao, Ch. Woodward. The honeycomb model of $\operatorname{gl}_{n}(\mathbb{C})$ tensor products. II. Puzzles determine facets of the Littlewood-Richardson cone. J. Amer. Math. Soc., 17(1): 19-48 (2004).

[Kwo09] J. Kwon. Differential operators and crystals of extremal weight modules. Adv. Math., 222(4): 1339-1369 (2009).

[Kwo10] J. Kwon. Littlewood-Richardson coefficients and weight crystals. Preprint, http://www.kurims.kyoto-u.ac.jp/ kyodo/kokyuroku/contents/pdf/1689-07. pdf (2010).

[LM06] J. De Loera, T. McAllister. On the computation of Clebsch-Gordan coefficients and the dilation effect. Exp. Math. 15(1): 7-20 (2006).

[Mac15] I.G. Macdonald. Symmetric functions and Hall polynomials. Oxford university press, (2015).

[MS01a] K. Mulmuley and M. Sohoni. Geometric complexity theory, P vs. NP and explicit obstructions. Advances in algebra and geometry, 239-261 (2001).

[MS01b] K. Mulmuley, M. Sohoni. Geometric complexity theory: an approach to the P vs. NP and related problems. SIAM J. Comput., 31(2): 496-526 (2001). 
[MS05] K. Mulmuley, M. Sohoni. Geometric complexity theory III, on deciding positivity of Littlewood-Richardson coefficients. Preprint, https://arxiv.org/pdf/cs/ 0501076.pdf (2005)

[MS07] K. Mulmuley, M. Sohoni. Geometric complexity theory: Introduction. Lecture notes, https://arxiv.org/pdf/0709.0746.pdf (2007).

[MS08] K. Mulmuley, M. Sohoni. Geometric complexity theory II: Towards explicit obstructions for embeddings among class varieties. SIAM J. Comput. 38(3): 1175-1206 (2008).

[MS17] K. Mulmuley, M. Sohoni. Geometric complexity theory V: on deciding nonvanishing of a generalized Littlewood-Richardson coefficient. J. Amer. Math. Soc. 30(1): 225-309 (2017).

[MNS12] K. Mulmuley, H. Narayanan, M. Sohoni. Geometric complexity theory III: on deciding nonvanishing of a Littlewood-Richardson coefficient. J. Algebr. Comb. 36(1): 103-110 (2012).

[Mul10] K. Mulmuley. Explicit Proofs and The Flip. Preprint https://arxiv.org/pdf/ arXiv:1009.0246 (2010).

[Mul11] K. Mulmuley. Geometric complexity theory VI: The flip via positivity. Technical report, The Computer Science Department, The University of Chicago (2011).

[Nar05] H. Narayanan. The computation of Kostka numbers and LittlewoodRichardson coefficients is \#P-complete. Preprint, https://arxiv.org/pdf/math/ 0501176.pdf (2005).

[Nar06] H. Narayanan. On the complexity of computing Kostka numbers and Littlewood-Richardson coefficients. J. Algebraic Combin., 24(3): 347-354 (2006).

[Ras04] E. Rassart. A polynomiality property for Littlewood-Richardson coefficients. J. Combin. theory. Ser. A, 107(2): 161-179 (2004).

[Rin76] C. M. Ringel. Representations of $K$-species and bimodules, J. Algebra 41: 269-302 (1976).

[Sag01] B. Sagan. The symmetric group: representations, combinatorial algorithms, and symmetric functions. Graduate Texts in Mathematics, volume 203 (2001).

[SB01] A. Schofield, M. van den Bergh. Semi-invariants of quivers for arbitrary dimension vectors. Indag. Math. (N.S.), 12(1): 125-138 (2001).

[Sch91] A. Schofield. Semi-invariants of quivers. J. London Math. Soc. (2), 43(3): 385-395 (1991).

[Sch92] A. Schofield. General representations of quivers. Proc. London Math. Soc. (3), 65(1): 46-64 (1992).

[Sch03] A. Schrijver. Combinatorial Optimization. Polyhedra and efficiency. Springer (2003). 
[She15] C. Sherman. Geometric proof of a conjecture of King, Tollu, and Toumazet. Preprint, https://arxiv.org/pdf/1505.06551.pdf (2015).

[She17] C. Sherman. Quiver generalization of a conjecture of King, Tollu, and Toumazet. J. Algebra, 480: 487-504 (2017).

[Ste87] J.R. Stembridge. Rational tableaux and the tensor algebra of $\mathrm{gl}_{n}$. J. of Combin. Theory, Ser. A. 46: 79-120 (1987).

[Tar86] É. Tardos. A strongly polynomial algorithm to solve combinatorial linear programs. Oper. Res., 34(2): 250-256 (1986).

[Wey12] H. Weyl Das asymtotische Verteilungsgesetz der Eigenwerte lineare partieller Differential gleichungen. Math. Ann., 71: 441-479 (1912).

[Ze199] A. Zelevinsky. Littlewood-Richardson semigroups. New Perspectives in Geometric Combinatorics 38: 337-345 (1999). 


\section{VITA}

Brett Collins was born in 1989 and raised in Licking, Missouri. He graduated with a B.A. from Truman State University in Kirksville, Missouri in 2011 before teaching at Parkland Community College for a year in Urbana-Champaign, Illinois. The following year he began his graduate studies at the University of Missouri-Columbia, earning his M.A. in 2014 and Ph.D. in 2018. After being in Missouri almost all of his life, he begins his trek eastward to Massachusetts to begin a Temporary Instructorship at Fitchburg State University in August 2018. 